\title{
TILING DIRECTED GRAPHS WITH TOURNAMENTS
}

\author{
ANDRZEJ CZYGRINOW ${ }^{1}$, LOUIS DEBIASIO ${ }^{2}$, THEODORE MOLLA ${ }^{3}$ and \\ ANDREW TREGLOWN ${ }^{4}$ \\ ${ }^{1}$ School of Mathematical and Statistical Sciences, Arizona State University, Tempe, \\ AZ 85281, USA; \\ email: aczygri@asu.edu \\ 2 Department of Mathematics, Miami University, Oxford, OH 45056, USA; \\ email: debiasld@miamioh.edu \\ ${ }^{3}$ Department of Mathematics and Statistics, University of South Florida, Tampa, FL 33620, USA; \\ email:molla@usf.edu \\ ${ }^{4}$ School of Mathematics, University of Birmingham, Edgbaston, Birmingham, B15 2TT, UK; \\ email: a.c.treglown@bham.ac.uk
}

Received 21 April 2016; accepted 9 January 2018

\begin{abstract}
The Hajnal-Szemerédi theorem states that for any positive integer $r$ and any multiple $n$ of $r$, if $G$ is a graph on $n$ vertices and $\delta(G) \geqslant(1-1 / r) n$, then $G$ can be partitioned into $n / r$ vertex-disjoint copies of the complete graph on $r$ vertices. We prove a very general analogue of this result for directed graphs: for any positive integer $r$ with $r \neq 3$ and any sufficiently large multiple $n$ of $r$, if $G$ is a directed graph on $n$ vertices and every vertex is incident to at least $2(1-1 / r) n-1$ directed edges, then $G$ can be partitioned into $n / r$ vertex-disjoint subgraphs of size $r$ each of which contain every tournament on $r$ vertices (the case $r=3$ is different and was handled previously). In fact, this result is a consequence of a tiling result for standard multigraphs (that is multigraphs where there are at most two edges between any pair of vertices). A related Turán-type result is also proven.
\end{abstract}

2010 Mathematics Subject Classification: 5C35 (primary); 5C20, 5C70 (secondary)

\section{Introduction}

1.1. Tilings in graphs. Given two (di)graphs $H$ and $G$, an $H$-tiling in $G$ is a collection of vertex-disjoint copies of $H$ in $G$. An $H$-tiling is called perfect if it covers all the vertices of $G$. Perfect $H$-tilings are also referred to as $H$-factors

(c) The Author(s) 2018. This is an Open Access article, distributed under the terms of the Creative Commons Attribution licence (http://creativecommons.org/licenses/by/4.0/), which permits unrestricted re-use, distribution, and reproduction in any medium, provided the original work is properly cited. 
or perfect $H$-packings. If $H$ is connected and of order at least three, the problem of deciding whether a graph $G$ contains a perfect $H$-tiling is NP-complete [11]. In light of this, it is natural to ask for simple sufficient conditions which force a graph to contain a perfect $H$-tiling.

A cornerstone result in extremal graph theory is the following theorem of Hajnal and Szemerédi [9].

THEOREM 1.1 (Hajnal and Szemerédi [9]). Every graph $G$ whose order $n$ is divisible by $r$ and whose minimum degree satisfies $\delta(G) \geqslant(1-1 / r) n$ contains a perfect $K_{r}$-tiling.

Notice that the minimum degree condition in Theorem 1.1 is tight. Earlier, Corrádi and Hajnal [5] proved Theorem 1.1 in the case when $r=3$. More recently, Kierstead and Kostochka [15] gave a short proof of the Hajnal-Szemerédi theorem.

Over the last three decades there has been much work on generalizing the Hajnal-Szemerédi theorem. One highlight in this direction is a result of Kühn and Osthus $[19,20]$ that characterizes, up to an additive constant, the minimum degree which ensures that a graph $G$ contains a perfect $H$-tiling for an arbitrary graph $H$. Other notable results include an Ore-type analogue of the Hajnal-Szemerédi theorem of Kierstead and Kostochka [14] and an r-partite version of the HajnalSzemerédi theorem of Keevash and Mycroft [12]. See [21] for a survey including many of the results on graph tiling.

There has also been interest in tiling problems for directed graphs and hypergraphs. A recent survey of Zhao [31] gives an extensive overview of the latter problem. In this paper we prove a directed analogue of the HajnalSzemerédi theorem.

1.2. Tilings in directed graphs. Throughout this paper, the digraphs we consider do not have loops and we allow for at most one edge in each direction between any pair of vertices. An oriented graph is a digraph without 2-cycles.

For digraphs there is more than one natural notion of degree: The minimum semidegree $\delta^{0}(G)$ of a digraph $G$ is the minimum of its minimum outdegree $\delta^{+}(G)$ and its minimum indegree $\delta^{-}(G)$. The minimum degree $\delta(G)$ of $G$ is the minimum number of edges incident to a vertex in $G$.

For oriented graphs, there has been some progress on obtaining degree conditions that force tilings. Denote by $T_{r}$ the transitive tournament of $r$ vertices and by $C_{3}$ the cyclic triangle. Yuster [30] observed that an oriented graph $G$ on $n \in 3 \mathbb{N}$ vertices and with $\delta(G) \geqslant 5 n / 6$ contains a perfect $T_{3}$-tiling (and also gave 
a minimum degree condition which forces a perfect $T_{r}$-tiling for $r>3$ ). More recently, Balogh et al. [2] determined the minimum semidegree threshold which ensures a perfect $T_{3}$-tiling in an oriented graph, thereby resolving a conjecture from [26]. Keevash and Sudakov [13] showed that every oriented graph $G$ on $n$ vertices with $\delta^{0}(G) \geqslant(1 / 2-o(1)) n$ contains a $C_{3}$-tiling covering all but at most 3 vertices. (There are examples that show even $\delta^{0}(G) \geqslant(n-3) / 2$ does not guarantee a perfect $C_{3}$-tiling.)

Denote by $\mathcal{T}_{r}$ the set of all tournaments on $r$ vertices. Let $T \in \mathcal{T}_{r}$. For digraphs, the minimum semidegree threshold that forces a perfect $T$-tiling was characterized in [27].

THEOREM 1.2 [27]. Given an integer $r \geqslant 3$, there exists an $n_{0} \in \mathbb{N}$ such that the following holds. Suppose $T \in \mathcal{T}_{r}$ and $G$ is a digraph on $n \geqslant n_{0}$ vertices where $r$ divides $n$. If

$$
\delta^{0}(G) \geqslant(1-1 / r) n
$$

then $G$ contains a perfect $T$-tiling.

Notice that the minimum semidegree condition in Theorem 1.2 is tight. Note also that Theorem 1.2 implies the Hajnal-Szemerédi theorem for large graphs. An earlier result of Czygrinow et al. [7] gives an asymptotic version of Theorem 1.2 for perfect $C_{3}$-tilings.

It is natural to ask whether Theorem 1.2 can be strengthened by replacing the minimum semidegree condition with a minimum degree condition. In particular, can one replace the minimum semidegree condition in Theorem 1.2 with $\delta(G) \geqslant$ $2(1-1 / r) n-1$ ? However, when $T=C_{3}$ the answer is no. Indeed, an example of Wang [29] shows that $\delta(G) \geqslant(3 n-5) / 2$ does not ensure a perfect $C_{3}$-tiling. On the other hand, he showed that minimum degree $\delta(G) \geqslant(3 n-3) / 2$ does force a perfect $C_{3}$-tiling in a digraph $G$. This led to the following question being raised in [27].

QUESTION 1.3. Let $n, r \in \mathbb{N}$ such that $r$ divides $n$. Let $T \in \mathcal{T}_{r} \backslash\left\{C_{3}\right\}$. Does every digraph $G$ on $n$ vertices with $\delta(G) \geqslant 2(1-1 / r) n-1$ contain a perfect $T$-tiling?

Czygrinow et al. [6] answered Question 1.3 in the affirmative for perfect $T_{r}$ tilings and also in the case when $r$ is sufficiently large and $\delta(G) \geqslant 2(1-1 / r+$ $o(1)) n$.

The main result of this paper gives an exact solution to a stronger version of Question 1.3 for all $r \geqslant 4$. 
THEOREM 1.4. Given an integer $r \geqslant 4$, there exists an $n_{0} \in \mathbb{N}$ such that the following holds. Suppose $G$ is a digraph on $n \geqslant n_{0}$ vertices where $r$ divides $n$. If

$$
\delta(G) \geqslant 2(1-1 / r) n-1,
$$

then $G$ contains $n / r$ vertex-disjoint subdigraphs each of which contains every tournament on $r$ vertices.

The following theorem from [7], in some sense, answers the analogous question when $r=3$.

THEOREM 1.5 [7]. Suppose $G$ is a digraph on $n$ vertices where 3 divides $n$. If $\delta(G) \geqslant 4 n / 3-1$, then there exist $n / 3$ vertex-disjoint subdigraphs each of size 3 such that each subdigraph contains a $T_{3}$ and all but at most one contains $C_{3}$ as well.

This is best possible in the following two senses: (i) there exist digraphs $G$ for which $\delta(G)=4 n / 3-2$ and which do not contain a triangle factor of any kind and; (ii) by Wang's example in [29], there exist digraphs such that $\delta(G) \geqslant 4 n / 3-1$ that do not have a perfect $C_{3}$-tiling. However, there is perhaps more to say about the case when $r=3$, as the following conjecture, which originally appeared in [23], suggests.

ConjeCture 1.6 [23]. Suppose $G$ is a digraph on $n$ vertices where 3 divides $n$. If $\delta(G) \geqslant 4 n / 3-1$ and $G$ is strongly 2-connected, then there exist $n / 3$ vertexdisjoint subdigraphs such that each of these subdigraphs contain both $T_{3}$ and $C_{3}$.

It should be noted that in [7] Conjecture 1.6 was proven when $\delta(G) \geqslant(3 n-$ $3) / 2$. Note that when $\delta(G) \geqslant(3 n-3) / 2, G$ is strongly 2 -connected.

1.3. Tilings in multigraphs. Instead of proving Theorem 1.4 directly, we prove a more general result concerning tilings in multigraphs. A similar approach was taken in [7] and [6].

Suppose that $M$ is a multigraph. The minimum degree $\delta(M)$ of $M$ is the minimum number of edges incident to a vertex in $M$. For $x, y \in V(M)$ we write $\mu(x y)$ to denote the number of edges between $x$ and $y$ in $M$. We say that a loopless multigraph $M$ is standard if $\mu(x y) \leqslant 2$ for all $x, y \in V(M)$. Given vertices $x, y$ in a standard multigraph $M$ we say that $x y$ is a light edge if $\mu(x y)=1$ and a heavy edge if $\mu(x y)=2$.

Given a digraph $G$, the underlying multigraph $M$ of $G$ is the standard multigraph obtained from $G$ by ignoring the orientations of edges. Given a 
standard multigraph $M$, an orientation of the edges is legal if the resulting graph $G$ is a digraph (that is there is at most one edge in each direction between any pair of vertices in $G$ ). A standard multigraph $M$ on $r$ vertices is universal if, given any legal orientation $G$ of $M$, we have that $T \subseteq G$ for every $T \in \mathcal{T}_{r}$. For example, let $M$ be a standard multigraph on $r$ vertices where $\mu(x y)=1$, 2 for all distinct $x, y \in V(M)$ and the collection of light edges in $M$ forms a matching. Then $M$ is universal. On the other hand if $M$ is a standard multigraph on $n$ vertices that contains a cycle on light edges then $M$ is not universal. (There is a legal orientation of $M$ without a copy of $T_{r}$.) Write $\mathcal{U}_{r}$ for the set of all universal standard multigraphs on $r$ vertices.

Throughout the paper, instead of dealing with the set $\mathcal{U}_{r}$ itself, we mainly work with three subsets of $\mathcal{U}_{r}: \overline{\mathcal{K}}_{r}, \hat{\mathcal{K}}_{r}$ and $\mathcal{K}_{r}^{\prime}$. The elements of each of these three subsets are obtained from the complete standard multigraph on $r$ vertices by removing the edges from a set of vertex-disjoint (light) paths $P_{1}, \ldots, P_{t}$ where $\left|P_{1}\right| \geqslant\left|P_{2}\right| \geqslant \cdots \geqslant\left|P_{t}\right|$. The elements of $\overline{\mathcal{K}}_{r}$ are formed by removing the edges of $P_{1}, \ldots, P_{t}$ from the complete standard multigraph on $r$ vertices where we stipulate that $\left|P_{i}\right| \leqslant 2$ for all $i \in[t]$, that is the elements of $\overline{\mathcal{K}}_{r}$ are formed by removing a (light) matching from the complete standard multigraph on $r$ vertices. The elements of $\hat{\mathcal{K}}_{r}$ are formed in the same way, but we stipulate that $\left|P_{1}\right| \leqslant 3$ and $\left|P_{i}\right| \leqslant 2$ for all $i \geqslant 2$. To form elements of $\mathcal{K}_{r}^{\prime}$, we stipulate that either $\left|P_{1}\right| \leqslant 4$ and $\left|P_{i}\right| \leqslant 2$ for all $i \geqslant 2$, or $\left|P_{1}\right|,\left|P_{2}\right| \leqslant 3$ and $\left|P_{i}\right| \leqslant 2$ for all $i \geqslant 3$.
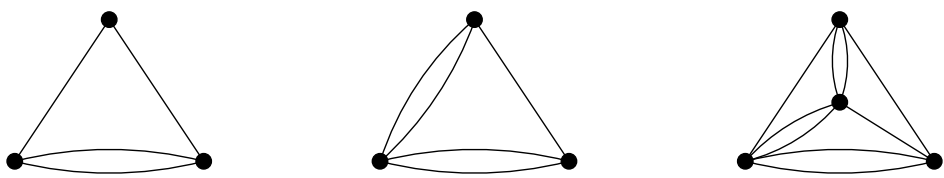

Figure 1. On the left is an element of $\hat{\mathcal{K}}_{3}$ which is not in $\mathcal{U}_{3}$. In the middle is an element of $\overline{\mathcal{K}}_{3}$ which is in $\mathcal{U}_{3}$. On the right is an element of $\mathcal{K}_{4}^{\prime}$ which is in $\mathcal{U}_{4}$.

We now prove that $\overline{\mathcal{K}}_{r} \subseteq \hat{\mathcal{K}}_{r} \subseteq \mathcal{K}_{r}^{\prime} \subseteq \mathcal{U}_{r}$ for $r \geqslant 4$. While this follows from a very strong result of Havet and Thomassé [10], which states that every tournament $T$ on $n$ vertices contains every oriented path $P$ on $n$ vertices except when $P$ is the anti-directed path and $n \in\{3,5,7\}$, we prove it directly as we do not need the full strength of their result.

PROPOSITION 1.7. For $r \geqslant 2, \overline{\mathcal{K}}_{r} \subseteq \mathcal{U}_{r}$ and for $r \geqslant 4, \overline{\mathcal{K}}_{r} \subseteq \hat{\mathcal{K}}_{r} \subseteq \mathcal{K}_{r}^{\prime} \subseteq \mathcal{U}_{r}$.

Proof. Let $r \geqslant 2, T$ be a tournament on $r$ vertices, and let $\vec{K}$ be a legal orientation of $K \in \overline{\mathcal{K}}_{r}$, where $\vec{K}$ has exactly $t \leqslant\lfloor r / 2\rfloor$ light edges. Form a bijection from 
$V(T)$ to $V(\vec{K})$ by choosing $t$ independent edges in $T$ and mapping their endpoints to the light edges of $\vec{K}$ with the correct orientation. Then complete the bijection by mapping all other vertices of $T$ to $V(\vec{K})$ arbitrarily. Since all other edges of $\vec{K}$ are double edges, we have $T \subseteq \vec{K}$.

Let $r \geqslant 4, T$ be a tournament on $r$ vertices, and let $\vec{K}$ be a legal orientation of $K \in \mathcal{K}_{r}^{\prime}$, where the light edges of $\vec{K}$ form $t$ vertex-disjoint paths $P_{1}, \ldots$, $P_{t}$ with $\left|P_{1}\right| \geqslant \cdots \geqslant\left|P_{t}\right|$ where either $\left|P_{1}\right| \leqslant 4$ and $\left|P_{i}\right| \leqslant 2$ for all $i \geqslant 2$, or $\left|P_{1}\right|,\left|P_{2}\right| \leqslant 3$ and $\left|P_{i}\right| \leqslant 2$ for all $i \geqslant 3$. The statement follows from the following two facts which are straightforward to verify: (1) every tournament on 4 vertices contains every orientation of a path on 4 vertices; (2) every tournament on 6 vertices contains two vertex-disjoint transitive triangles. We use this to first find an isomorphic copy of $P_{1}$ and $P_{2}$ (if applicable) in $T$, then we complete the embedding as in the first paragraph.

Given a collection of (multi)graphs $\mathcal{X}$, an $\mathcal{X}$-tiling in a (multi)graph $M$ is a collection of vertex-disjoint copies of elements of $\mathcal{X}$ in $M$. An $\mathcal{X}$-tiling is called perfect if it covers all the vertices of $M$. We refer to the elements of an $\mathcal{X}$-tiling as tiles. The next result (originally conjectured in [6]) ensures a standard multigraph of high minimum degree contains a perfect $\mathcal{U}_{r}$-tiling.

THEOREM 1.8. Given an integer $r \geqslant 2$, there exists an $n_{0} \in \mathbb{N}$ such that the following holds. Suppose $M$ is a standard multigraph on $n \geqslant n_{0}$ vertices where $r$ divides $n$. If

$$
\delta(M) \geqslant 2(1-1 / r) n-1
$$

then $M$ contains a perfect $\mathcal{K}_{r}^{\prime}$-tiling; so in particular, when $r \geqslant 4$, M contains a perfect $\mathcal{U}_{r}$-tiling.

Notice that Theorem 1.8 implies Theorem 1.4. Also note that, for every even $n$, Theorem 1.8 is true when $r=2$, because every simple graph on $n$ vertices with minimum degree at least $n / 2$ contains a perfect matching. When $r=3$, Theorem 1.8 was proved for every $n$ divisible by 3 in [7].

There are four different examples which show that the minimum degree condition in Theorem 1.8 is tight. Let $M_{1}$ and, when $r \geqslant 3$, let $M_{2}$ be the standard multigraphs on $n$ vertices which contain all possible edges except that $M_{1}$ contains an independent set $U$ of size $n / r+1$ and $M_{2}$ contains a set $U$ of size $2 n / r+1$ such that between any two distinct vertices $u, v \in U, \mu(u v)=1$. For both $i=1$, 2, $\delta\left(M_{i}\right)=2(1-1 / r) n-2$, but $M_{i}$ does not contain a perfect $\mathcal{U}_{r}$-tiling. In the case of $M_{1}$, this is because every element in $\mathcal{U}_{r}$ has at most 1 vertex in $U$ and in the case of $M_{2}$, it is because every element in $\mathcal{U}_{r}$ has at most 2 vertices in $U$. 

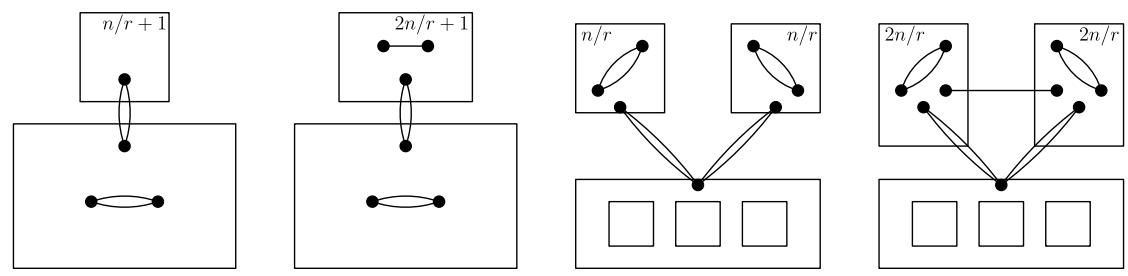

Figure 2. From left to right, the tightness examples $M_{1}, M_{2}, M_{3}, M_{4}$.

Suppose $n / r$ is odd. We define the standard multigraph $M_{3}$ on $n$ vertices as follows: Take two disjoint sets $X, Y$ of size $n / r$. Inside the sets $X, Y$ place all heavy edges, and between $X$ and $Y$ place no edges. From $X \cup Y$ to the remaining vertices, place all heavy edges. Now partition the remaining $(1-2 / r) n$ vertices into sets of size $n / r$ or $2 n / r$. Between all such sets, place all heavy edges. Inside the sets of size $2 n / r$ place all light edges and inside the sets of size $n / r$ place no edges. We have $\delta\left(M_{3}\right)=2(1-1 / r) n-2$. If $M_{3}$ contained a perfect $\mathcal{U}_{r}$-tiling, each copy of $\mathcal{U}_{r}$ would intersect the sets of size $n / r$ from $V\left(M_{3}\right) \backslash(X \cup Y)$ in exactly one vertex and the sets of size $2 n / r$ in exactly two vertices, and furthermore every copy of $\mathcal{U}_{r}$ has exactly 2 vertices from $X$ or exactly 2 vertices from $Y$. However, since $|X|$ and $|Y|$ are odd, $M_{3}$ does not contain a perfect $\mathcal{U}_{r}$-tiling.

Suppose $n / r$ is odd and $r \geqslant 4$. We form the standard multigraph $M_{4}$ on $n$ vertices similarly: Take two disjoint sets $X, Y$ of size $2 n / r$. Inside the sets $X, Y$ place all heavy edges and between $X$ and $Y$, place all light edges. From $X \cup Y$ to the remaining vertices, place all heavy edges. Now partition the remaining $(1-4 / r) n$ vertices into sets of size $n / r$ or $2 n / r$. Between all such sets, place all heavy edges. Inside the sets of size $2 n / r$ place all light edges and inside the sets of size $n / r$ place no edges. We have $\delta\left(M_{4}\right)=2(1-1 / r) n-2$. Now consider the standard multigraph $S_{r}$ with $r$ vertices in which there are all possible edges except that there is a vertex with precisely three light neighbours. If $r=4,5$ it is easy to check that $S_{r}$ is not universal (however, it is universal for $r>5$ ). With this in mind, suppose that $r=4,5$. Then if $M_{4}$ contained a perfect $\mathcal{U}_{r}$-tiling, each copy of $\mathcal{U}_{r}$ would intersect the sets of size $n / r$ from $V\left(M_{4}\right) \backslash(X \cup Y)$ in exactly one vertex and the sets of size $2 n / r$ in exactly two vertices, and furthermore every copy of $\mathcal{U}_{r}$ has exactly 4 vertices from $X$ or exactly 4 vertices from $Y$. However, since $|X|$ and $|Y|$ are not divisible by $4, M_{4}$ does not contain a perfect $\mathcal{U}_{r}$-tiling. Note that if $r>5$ then $M_{4}$ actually does contain a perfect $\mathcal{U}_{r}$-tiling, so $M_{4}$ is only an extremal example for the $\mathcal{U}_{r}$-tiling problem when $r=4$, 5. On the other hand, notice that for all $r \geqslant 4, M_{4}$ does not contain a perfect $\mathcal{K}_{r}^{\prime}$-tiling. So $M_{4}$ is an extremal example for the perfect $\mathcal{K}_{r}^{\prime}$-tiling problem for every $r \geqslant 4$. 
Finally, in the proof of Theorem 1.8 we (implicitly) produce a perfect $\mathcal{K}_{r}^{\prime}$-tiling where most of the tiles are elements from $\overline{\mathcal{K}}_{r}$. The following example, however, demonstrates that we need the minimum degree to be greater than $2(1-1 / r) n$ to guarantee a perfect $\overline{\mathcal{K}}_{r}$-tiling. For any $k \in \mathbb{N}$ and $r \geqslant 3$, let $G$ be a standard multigraph containing $n:=2 r k$ vertices constructed in the following way. Let $\left\{U_{1}, \ldots, U_{r-1}\right\}$ be a partition of $V(G)$ such that $\left|U_{1}\right|=3 k+1,\left|U_{2}\right|=3 k-1$ and $\left|U_{i}\right|=2 k$ for $i \in\{3, \ldots, r-1\}$. Place all possible light edges between $U_{1}$ and $U_{2}$, and for all other pairs of distinct sets $U_{i}$ and $U_{j}$, place all possible heavy edges. Also, inside both $G\left[U_{1}\right]$ and $G\left[U_{2}\right]$ place all possible heavy edges. The minimum degree of $G$ is $2(2 r k-2 k)=2(1-1 / r) n$, which is witnessed by any $v \in U_{3} \cup \cdots \cup U_{r-1}$. Now suppose that $G$ contains $2 k$ vertex-disjoint elements from $\overline{\mathcal{K}}_{r}$. Each one of these elements must have exactly 1 vertex in each of $U_{3}$, $\ldots, U_{r-1}$ and exactly 3 vertices in $G\left[U_{1} \cup U_{2}\right]$; however, $G\left[U_{1} \cup U_{2}\right]$ does not contain a perfect $\overline{\mathcal{K}}_{3}$-tiling. Note that when $r=3$, this corresponds to Wang's example in [29].

\section{Overview of the proof of Theorem $\mathbf{1 . 8}$}

2.1. The overall approach. As with many proofs in the area, the proof of Theorem 1.8 divides into extremal and nonextremal cases. Roughly speaking, in the extremal case we consider those standard multigraphs that are 'close' to the extremal examples $M_{1}, M_{2}, M_{3}$ and $M_{4}$ that were introduced after the statement of Theorem 1.8. We deal with these extremal cases in one unified approach in Section 10.

Suppose that $G$ is as in Theorem 1.8. Further, suppose that there is a 'small' set $M \subseteq V(G)$ with the property that both $G[M]$ and $G[M \cup Q]$ contain perfect $\mathcal{U}_{r}$-tilings for any 'very small' set $Q \subseteq V(G)$ where $|Q| \in r \mathbb{N}$. Then notice that, to find a perfect $\mathcal{U}_{r}$-tiling in $G$, it suffices to find an 'almost' perfect $\mathcal{U}_{r}$-tiling in $G^{\prime}:=G \backslash M$. Indeed, suppose that $G^{\prime}$ contains a $\mathcal{U}_{r}$-tiling $\mathcal{M}_{1}$ covering all but a very small set of vertices $Q$. Then by definition of $M, G[M \cup Q]$ contains a perfect $\mathcal{U}_{r}$-tiling $\mathcal{M}_{2}$. Thus, $\mathcal{M}_{1} \cup \mathcal{M}_{2}$ is a perfect $\mathcal{U}_{r}$-tiling in $G$, as desired.

Roughly speaking, we refer to such a set $M$ as an 'absorbing set' (see Section 8 for the precise definition of such a set). The 'absorbing method' was first used in [24] and has subsequently been applied to numerous embedding problems in extremal graph theory.

In general, a multigraph $G$ as in Theorem 1.8 may not contain an absorbing set. Indeed, consider the multigraph $G$ with disjoint vertex classes $V_{1}, \ldots, V_{r}$ each of size $n / r$ in which there are all possible heavy edges except that each $V_{i}$ is an independent set. Then if $Q$ is any set of $r$ vertices in $V_{1}$, there is no 
set $M \subseteq V(G)$ such that both $G[M]$ and $G[M \cup Q]$ contain perfect $\mathcal{U}_{r}$-tilings. Note that this multigraph is close to the extremal example $M_{1}$. It turns out that when $G$ is nonextremal, we can always find an absorbing set $M$; we construct this set in Section 8.

Thus, to complete the proof in the nonextremal case we must find an 'almost' perfect $\mathcal{U}_{r}$-tiling in $G \backslash M$. Actually in Section 6 we prove a result that ensures any multigraph $G$ as in Theorem 1.8 contains a $\overline{\mathcal{K}}_{r}$-tiling covering almost all the vertices of $G$, see Corollary 6.6. However, this does not quite guarantee a large enough $\mathcal{U}_{r}$-tiling in $G \backslash M$. Indeed, the leftover set $Q$ obtained by applying Corollary 6.6 to $G \backslash M$ will be slightly larger than the absorbing set $M$, and thus $M$ will not be able to absorb $Q$.

To overcome this we again have to use the property that our multigraph $G$ is nonextremal. Using the $\overline{\mathcal{K}}_{r}$-tiling obtained from Corollary 6.6 we build a significantly bigger $\mathcal{K}_{r}^{\prime}$-tiling so that now the leftover set is very small compared to $M$. This is another delicate part of the proof and is dealt with in Section 7.

In Section 6 we apply a Turán-type result for standard multigraphs; this is introduced in Section 4 (see Theorem 4.2). We also introduce a multigraph regularity lemma in Section 5 and make use of this in Sections 6 and 7.

2.2. Extremal versus nonextremal graphs. Notice that both $M_{1}$ and $M_{2}$ have the property that they contain (i) an independent set of size $n / r$ or (ii) a set of vertices of size $2 n / r$ which contains no heavy edge. Further, $M_{3}$ and $M_{4}$ have this property when $r \geqslant 3$ and $r \geqslant 5$, respectively. These properties turn out to be barriers for obtaining an absorbing set. Thus, as suggested above, we deal with the case when our multigraph $G$ is 'close' to satisfying (i) and/or (ii) separately. More precisely, given $\gamma>0$ and $r \in \mathbb{N}$, we say that a multigraph $G$ on $n$ vertices is $(1 / r, \gamma)$-extremal if:

(i) there exists $S \subseteq V(G)$ such that ||$S|-n / r|<\gamma n$ and $e(G[S])<\gamma n^{2}$; or

(ii) there exists $S \subseteq V(G)$ such that ||$S|-2 n / r|<\gamma n$ and $e_{2}(G[S])<\gamma n^{2}$.

(Here we write $e_{2}(G[S])$ to denote the number of heavy edges in $G[S]$.) Note that $M_{3}$ and $M_{4}$ satisfy neither (i) nor (ii) when $r=2$ and $r=4$, respectively. When $r=2, M_{3}$ contains two vertex classes $X, Y$ of size $n / 2$ for which there are no edges between $X$ and $Y$. Similarly, when $r=4, M_{4}$ contains two vertex classes $X, Y$ of size $n / 2$ for which there are no heavy edges between $X$ and $Y$. Standard multigraphs that are 'close' to satisfying one of these two properties will also be considered in the extremal case. More precisely, given $\gamma>0$ and $r=2,4$, a multigraph $G$ on $n$ vertices is $(r, \gamma)$-splittable if there exist disjoint sets $U_{1}, U_{2} \subseteq V(G)$ such that: 
- $\left|U_{1}\right|,\left|U_{2}\right| \geqslant(1 / 2-\gamma) n$; and either

- $r=2$ and there are at most $\gamma n^{2}$ edges between $U_{1}$ and $U_{2}$ in $G$; or

- $r=4$ and there are at most $\gamma n^{2}$ heavy edges between $U_{1}$ and $U_{2}$ in $G$.

To summarize, the extremal case thus considers multigraphs that are $(1 / r, \gamma)$ extremal, $(2, \gamma)$-splittable or $(4, \gamma)$-splittable. While now we are unable to employ the absorbing method, the multigraphs considered in the extremal case have a rigid structure that we can exploit. For example, if $G$ is $(4, \gamma)$-splittable and satisfies $\delta(G) \geqslant 3 n / 2-1$, then there is a partition $A, B$ of $V(G)$ such that there are almost all possible light edges between $A$ and $B$; and both $G[A]$ and $G[B]$ contain almost all possible heavy edges. The proof of the extremal case takes a similar approach to that of Komlós, Sárközy and Szemerédi in their proof of the Alon-Yuster conjecture [17].

2.3. Absorbing in the nonextremal case. In order to construct our desired absorbing set it suffices to show that, for every $x, y \in V(G)$ there are $\Theta\left(n^{r-1}\right)$ $(r-1)$-sets $X \subseteq V(G)$ such that both $G[X \cup\{x\}]$ and $G[X \cup\{y\}]$ contain $\mathcal{K}_{r}^{\prime}$. This is formalized by a result of Lo and Markström [22] (we state it as Lemma 8.1 in Section 8). Our following lemma thus yields an absorbing set in the nonextremal case.

LEMMA 2.1. Let $r \geqslant 3,0<1 / n \ll \eta, \phi \ll \gamma \ll 1 / r$, and let $G$ be a multigraph on $n$ vertices. If $\delta(G) \geqslant 2(1-1 / r-\eta) n$ and $G$ is not $(1 / r, \gamma)$-extremal and either $r \neq 4$ or $G$ is not $(4, \gamma)$-splittable, then for all distinct $x_{1}, x_{2} \in V(G)$ there exist at least $(\phi n)^{r-1}(r-1)$-sets $Y \subseteq V(G)$ such that $G\left[Y \cup\left\{x_{1}\right\}\right]$ and $G\left[Y \cup\left\{x_{2}\right\}\right]$ both contain $\mathcal{K}_{r}^{\prime}$.

We prove Lemma 2.1 in Section 8.

\section{Notation}

For the rest of the paper, when we write multigraph, we mean standard multigraph. Let $G$ be a multigraph. We write $e(G)$ for the total number of edges in $G$ and $e_{2}(G)$ for the number of heavy edges in $G$. Given a subset $X \subseteq V(G)$, we write $G[X]$ for the submultigraph of $G$ induced by $X$. We write $G \backslash X$ for the submultigraph of $G$ induced by $V(G) \backslash X$ and define $\bar{X}:=V(G) \backslash X$.

In a multigraph $G$, for $i=1,2$ let $N_{G}^{i}(v):=\{u: u v \in E(G)$ and $\mu(u v)=i\}$ and $d_{G}^{i}(v):=\left|N_{G}^{i}(v)\right|$. Let $N_{G}(v):=N_{G}^{1}(v) \cup N_{G}^{2}(v)$. We define the degree $d_{G}(v)$ of $v$ to be the sum of the multiplicities of the edges incident with $v$, that is 
$d_{G}(v):=d_{G}^{1}(v)+2 d_{G}^{2}(v)$. Note that $d_{G}(v)=\left|N_{G}(v)\right|+\left|N_{G}^{2}(v)\right|$. Given a set $X \subseteq V(G)$ (or subgraph $X$ of $G$ ) we write $d_{G}(v, X)$ for the total number of edges in $G$ incident to $v$ whose other endpoint lies in $X$ (or $V(X)$ ). We define $d_{G}^{2}(v, X)$ similarly. Given disjoint $X, Y \subseteq V(G)$ (or subgraphs $X, Y$ of $G$ ) we write $e_{G}(X, Y)$ for the total number of edges in $G$ with one endpoint in $X$ (or $V(X)$ ) and the other in $Y$ (or $V(Y)$ ); We write $e_{2}(X, Y)$ for the total number of heavy edges in $G$ with one endpoint in $X$ (or $V(X)$ ) and the other in $Y$ (or $V(Y)$ ), and let $E_{2}(X, Y)$ denote the set of all such edges. In all the aforementioned notation we omit the subscript $G$ if the multigraph is clear from the context.

When $\mathcal{U}$ is a family of multigraphs (digraphs) and $G$ is a multigraph (digraph) we write $\mathcal{U} \subseteq G$ to mean that some $U \in \mathcal{U}$ is a subgraph of $G$. If $U \in \mathcal{U}$ we say that $U$ is a copy of $\mathcal{U}$. If $\mathcal{U}$ is a family of multigraphs and $G$ is a digraph we write $\mathcal{U} \subseteq G$ to mean that there is a legal orientation $\vec{U}$ of some $U \in \mathcal{U}$ such that $\vec{U}$ is a subdigraph of $G$.

Given a graph $G$ we let $G(t)$ denote the graph obtain from $G$ by replacing each vertex $x \in V(G)$ with a set $V_{x}$ of $t$ vertices so that, for all $x, y \in V(G)$ :

- if $x \neq y$ then $V_{x} \cap V_{y}=\emptyset$;

- if $x y \in E(G)$ then there are all possible edges in $G(t)$ between $V_{x}$ and $V_{y}$;

- if $x y \notin E(G)$ then there are no edges in $G(t)$ between $V_{x}$ and $V_{y}$.

Similarly, given a multigraph $G$ we let $G(t)$ denote the multigraph obtain from $G$ by replacing each vertex $x \in V(G)$ with a set $V_{x}$ of $t$ vertices so that, for all $x, y \in V(G)$ :

- if $x \neq y$ then $V_{x} \cap V_{y}=\emptyset$;

- if $\mu(x y)=2$ in $G$ then there are all possible heavy edges in $G(t)$ between $V_{x}$ and $V_{y}$;

- if $\mu(x y)=1$ in $G$ then there are all possible light edges in $G(t)$ between $V_{x}$ and $V_{y}$;

- if $\mu(x y)=0$ in $G$ then there are no edges in $G(t)$ between $V_{x}$ and $V_{y}$.

Given a set $X$ we write, for example $X+v, X-v$ and $X+v-w$ for $X \cup\{v\}$, $X \backslash\{v\}$ and $(X \backslash\{w\}) \cup\{v\}$ respectively. Similarly given multigraphs $T, G$ where $T \subseteq G$ and $v, w \in V(G)$, we write, for example, $T+v, T-v, T+v-w$ for the multigraphs $G[V(T)+v], G[V(T)-v]$ and $G[V(T)+v-w]$ respectively. We define, for example, $T-X, T+X, T-X+Y$ similarly where $X, Y \subseteq V(G)$. 
Throughout the paper, we write $0<\alpha \ll \beta \ll \gamma$ to mean that we can choose the constants $\alpha, \beta, \gamma$ from right to left. More precisely, there are increasing functions $f$ and $g$ such that, given $\gamma$, whenever we choose $\beta \leqslant f(\gamma)$ and $\alpha \leqslant g(\beta)$, all calculations needed in our proof are valid. Hierarchies of other lengths are defined in the obvious way.

\section{Turán-type results for universal multigraphs and digraphs}

In this section we determine the density threshold that ensures a standard multigraph contains a universal graph, and therefore determine the threshold that forces a digraph to contain any tournament of a given size.

Let $t_{r-1}(n)$ be the maximum number of edges in an $(r-1)$-partite graph on $n$ vertices and let $T_{r-1}(n)$ be the $(r-1)$-partite graph that realizes this bound. Note that when $n \geqslant r-1$

$$
t_{r-1}(n)-t_{r-1}(n-(r-1))=\frac{(r-1)(r-2)}{2}+(r-2)(n-(r-1)),
$$

and for any $n$

$$
t_{r-1}(n) \leqslant\left(1-\frac{1}{r-1}\right) \frac{n^{2}}{2},
$$

with equality when $r-1$ divides $n$.

OBSERVATION 4.1. Let $D_{r-1}(n)$ be the digraph obtained by replacing every edge of $T_{r-1}(n)$ with two oppositely oriented directed edges and let $M_{r-1}(n)$ be the underlying multigraph of $D_{r-1}(n)$. Then $D_{r-1}(n)$ contains no tournament on $r$ vertices and $M_{r-1}(n)$ contains no graph on $r$ vertices whose underlying graph is complete.

Brown and Harary [4] proved that if a digraph $D$ on $n$ vertices contains more than $2 t_{r-1}(n)$ edges, then $D$ contains every tournament on $r$ vertices. The following theorem strengthens their result by showing that $D$ contains a subdigraph on $r$ vertices which itself contains every tournament on $r$ vertices; in fact, we prove an even more general result about multigraphs.

THEOREM 4.2. Let $r \geqslant 2$ and let $G$ be a multigraph on $n$ vertices. If $e(G)>$ $2 t_{r-1}(n)$, then $\overline{\mathcal{K}}_{r} \subseteq G$.

Proof. The proof proceeds by double induction on $r$ and $n$. Clearly the result holds for $r=2$. Let $r \geqslant 3$ and let $G$ be a standard multigraph on $n$ vertices such that $\overline{\mathcal{K}}_{r} \nsubseteq G$. If $n \leqslant r-1$, then $e(G) \leqslant 2 t_{r-1}(n)$; so suppose $n \geqslant r$. 
Since $2 t_{r-2}(n) \leqslant 2 t_{r-1}(n)<e(G)$, by induction, there exists a copy of $\overline{\mathcal{K}}_{r-1}$ in $G$; let $H$ be a copy of $\overline{\mathcal{K}}_{r-1}$ with the maximum number of edges. If there exists $v \in V(G) \backslash V(H)$ such that $d(v, H) \geqslant 2(r-1)-1$, then we can either add $v$ to $H$ to make a copy of $\overline{\mathcal{K}}_{r}$ or we can swap $v$ with a vertex in $H$ to increase the number of edges in $H$; either way, a contradiction. So for all $v \in V(G) \backslash V(H)$ we have $d(v, H) \leqslant 2(r-1)-2=2(r-2)$. Thus by (1) and induction on $n$,

$$
\begin{aligned}
e(G) & =e(H)+e(G-H, H)+e(G-H) \\
& \leqslant(r-1)(r-2)+2(r-2)(n-(r-1))+2 t_{r-1}(n-(r-1)) \\
& =2\left(\frac{(r-1)(r-2)}{2}+(r-2)(n-(r-1))+t_{r-1}(n-(r-1))\right) \\
& =2 t_{r-1}(n) .
\end{aligned}
$$

COROLlary 4.3. Let $G$ be a multigraph on $n$ vertices. If $\delta(G)>2(1-$ $1 /(r-1)) n$ or $e(G)>(1-1 /(r-1)) n^{2}$, then $\overline{\mathcal{K}}_{r} \subseteq G$.

First note that Theorem 4.2 and Corollary 4.3 immediately imply the analogous digraph versions. Observation 4.1 shows that the density conditions in Theorem 4.2 and Corollary 4.3 to force a copy of $\overline{\mathcal{K}}_{r}$ are best possible; however, one may wonder if the same density conditions could force a multigraph $K$ whose complement contains a matching on at most $\lfloor r / 2\rfloor-1$ light edges. The following observation shows that this is not the case.

OBSERVATION 4.4. Let $K$ be a multigraph on $r$ vertices such that the complement of $K$ is a matching with at most $\lfloor r / 2\rfloor-1$ light edges. If $r$ is even, let $n \in r \mathbb{N}$ and if $r$ is odd, let $n \in(r+1) \mathbb{N}$. For sufficiently large $n$, there exists a multigraph $G$ on $n$ vertices with (significantly) more than $2 t_{r-1}(n)$ edges for which $K \nsubseteq G$.

Proof. First suppose $r$ is even and $n=r k$. Let $G$ be an $r$ /2-partite multigraph with all parts of size $2 k=2 n / r$. Inside each part put all possible light edges and between the parts put all possible heavy edges. We have

$$
e(G)=n^{2}-n-\frac{r}{2}\left(\begin{array}{c}
2 n / r \\
2
\end{array}\right)=\left(1-\frac{1}{r}-\frac{1}{2 n}\right) n^{2},
$$

which is much larger than $(1-1 /(r-1)) n^{2} \geqslant 2 t_{r-1}(n)$.

Now suppose $r$ is odd and $n=(r+1) k$. Let $G$ be an $(r+1) / 2$-partite multigraph with all parts of size $2 k=2 n /(r+1)$. Inside each part put all possible light edges 
and between the parts put all possible heavy edges. We have

$$
e(G)=n^{2}-n-\frac{r+1}{2}\left(\begin{array}{c}
2 n /(r+1) \\
2
\end{array}\right)=\left(1-\frac{1}{r+1}-\frac{1}{2 n}\right) n^{2},
$$

which is much larger than $(1-1 /(r-1)) n^{2} \geqslant 2 t_{r-1}(n)$.

Note that in each case $G$ contains no copy of $K$.

Finally, we address the issue of the structure of $\overline{\mathcal{K}}_{r}$-free multigraphs with $2 t_{r-1}(n)$ edges. Let $\mathcal{D}_{2}^{*}(n)$ be the family of digraphs obtained by partitioning $n$ as $n=n_{1}+\cdots+n_{k}$ such that $n_{1}, \ldots, n_{k}$ are positive integers and at most one of the $n_{i}$ s is odd, and taking $k$ disjoint copies, $D_{1}, \ldots, D_{k}$ of $D_{2}\left(n_{1}\right), \ldots, D_{2}\left(n_{k}\right)$, then adding all edges directed from $D_{i}$ to $D_{j}$ for all $1 \leqslant i<j \leqslant k$. In particular, note that $D_{2}(n) \in \mathcal{D}_{2}^{*}(n)$. Brown and Harary [4] proved that if $T \in \mathcal{T}_{r}$ and $D$ is a $T$-free digraph on $n$ vertices with $2 t_{r-1}(n)$ edges, then $D \cong D_{r-1}(n)$ unless $T=C_{3}$ in which case $D \in \mathcal{D}_{2}^{*}(n)$. The following observation shows that in our case, there is a whole family of tightness examples. Let $\mathcal{M}_{2}^{*}(n)$ be the family of multigraphs underlying the digraphs in $\mathcal{D}_{2}^{*}(n)$.

OBSERVATION 4.5. Given $r \geqslant 4$, let $n \in r \mathbb{N}$ and let $\mathcal{M}_{r-1}^{*}(n)$ be the family of multigraphs on $n$ vertices which can be obtained from $M_{r-1}(n)$ by the following process. Take disjoint pairs of colour classes and replace each such pair with a copy of $M \in \mathcal{M}_{2}^{*}(2 n / r)$, leaving all other edges between the sets as they were. Then every $M \in \mathcal{M}_{r-1}^{*}(n)$ does not contain $\overline{\mathcal{K}}_{r}$.

Proof. Let $M \in \mathcal{M}_{r-1}^{*}(n)$, let $X_{1}, \ldots, X_{s}$ be the colour classes from $M$ which were not modified, and let $Y_{1}, \ldots, Y_{t}$ be the sets from $M$ which appeared as a result of merging two of the original colour classes. We have $r-1=s+2 t$ and thus any copy of $\overline{\mathcal{K}}_{r}$ must contain at least 2 vertices from some $X_{i}$, which is clearly not possible, or at least 3 vertices from some $Y_{j}$, which would imply that $Y_{j}$ contains a copy of $\overline{\mathcal{K}}_{3}$, which is not the case. Thus $\overline{\mathcal{K}}_{r} \notin M$.

It would be interesting to determine whether every $\overline{\mathcal{K}}_{r}$-free multigraph on $2 t_{r-1}(n)$ edges is a member of $\mathcal{M}_{r-1}^{*}(n)$, and more generally, whether every $\overline{\mathcal{K}}_{r}$ free multigraph on $2 t_{r-1}(n)-o\left(n^{2}\right)$ edges is sufficiently 'close' (in edit-distance) to some member of $\mathcal{M}_{r-1}^{*}(n)$.

\section{A regularity lemma for standard multigraphs}

In the proof of Theorems 1.8 and 7.2 we apply a version of Szemerédi's regularity lemma [25] for multigraphs. Before we state it we need some more 
definitions. The density of a bipartite graph $G=(A, B)$ with vertex classes $A$ and $B$ is defined to be

$$
d_{G}(A, B):=\frac{e_{G}(A, B)}{|A||B|} .
$$

We write $d(A, B)$ if this is unambiguous. Given any $\varepsilon>0$ we say that $G$ is $\varepsilon$ regular if for all $X \subseteq A$ and $Y \subseteq B$ with $|X|>\varepsilon|A|$ and $|Y|>\varepsilon|B|$ we have that $|d(X, Y)-d(A, B)|<\varepsilon$.

Given disjoint vertex sets $A$ and $B$ in a graph $G$, we write $(A, B)$ for the induced bipartite subgraph of $G$ whose vertex classes are $A$ and $B$. If $G$ is a multigraph and $A, B \subseteq V(G)$ are disjoint, then we write $(A, B)_{G}^{i}$ for the bipartite graph with vertex classes $A$ and $B$ where $a \in A$ and $b \in B$ are adjacent in $(A, B)_{G}^{i}$ precisely if $\mu(a b)=i$ in $G$.

The next well-known observation (see [16] for example) states that a large subgraph of a regular pair is also regular.

LEMMA 5.1. Let $0<\varepsilon<\alpha$ and $\varepsilon^{\prime}:=\max \{\varepsilon / \alpha, 2 \varepsilon\}$. Let $(A, B)$ be an $\varepsilon$-regular pair of densityd. Suppose $A^{\prime} \subseteq A$ and $B^{\prime} \subseteq B$ where $\left|A^{\prime}\right| \geqslant \alpha|A|$ and $\left|B^{\prime}\right| \geqslant \alpha|B|$. Then $\left(A^{\prime}, B^{\prime}\right)$ is an $\varepsilon^{\prime}$-regular pair with density $d^{\prime}$ where $\left|d^{\prime}-d\right|<\varepsilon$.

The following result will be applied in the proof of Theorem 6.1. It is (for example) a special case of [1, Corollary 2.3].

LEMmA 5.2. Let $\varepsilon, d>0$ and $m, r, t \in \mathbb{N}$ such that $0<1 / m \ll \varepsilon \ll d \ll 1 / r$ and $t \leqslant r$. Let $H$ be a graph obtained from $K_{r}$ by replacing every vertex of $K_{r}$ with $m$ vertices and replacing each edge of $K_{r}$ with an $\varepsilon^{2}$-regular pair of density at least $d$. Then $H$ contains a $K_{t}$-tiling covering all but at most $\varepsilon m r$ vertices.

We apply the following version of the regularity lemma, which is an immediate corollary of a 2-coloured regularity lemma from [3] (Theorem 2.4). This result in turn is easy to derive from the many-colour regularity lemma presented in [18] (Theorem 1.18).

LEMMA 5.3 (Degree form of multigraph regularity lemma). For any $\varepsilon>0$ and $M^{\prime} \in \mathbb{N}$, there exists $M=M\left(\varepsilon, M^{\prime}\right)$ such that the following holds. Let $G$ be a standard multigraph on $n$ vertices and let $0 \leqslant d \leqslant 1$. Then there exists a partition $\left\{V_{0}, V_{1}, \ldots, V_{k}\right\}$ of $V(G)$ with $M^{\prime} \leqslant k \leqslant M$ and a spanning subgraph $G^{\prime}$ of $G$ with the following properties: 
(i) $\left|V_{0}\right| \leqslant \varepsilon n$;

(ii) all clusters $V_{i}, i \in[k]$, are of the same size $((1-\varepsilon) / M) n \leqslant\left(n-\left|V_{0}\right|\right) / k=$ $\left|V_{1}\right| \leqslant n / M^{\prime}$

(iii) $d_{G^{\prime}}(v)>d_{G}(v)-(4 d+2 \varepsilon) n$ for all $v \in V\left(G^{\prime}\right)$;

(iv) $e\left(G^{\prime}\left[V_{i}\right]\right)=0$ for all $i \in[k]$;

(v) for all $1 \leqslant i<j \leqslant k$ and $c \in[2]$, the pair $\left(V_{i}, V_{j}\right)_{G^{\prime}}^{c}$ is E-regular with density either 0 or at least $d$.

We call $V_{1}, \ldots, V_{k}$ clusters, $V_{0}$ the exceptional set and $G^{\prime}$ the pure multigraph. Given a multigraph $G$, and parameters $\varepsilon, d, M^{\prime}$, we define the reduced multigraph $\Gamma$ as follows: Let $\left\{V_{0}, V_{1}, \ldots, V_{k}\right\}$ be the partition and $G^{\prime}$ be the subgraph of $G$ obtained from an application of Lemma 5.3 with parameters $\varepsilon, d, M^{\prime}$. We let $V(\Gamma)=\left\{V_{1}, \ldots, V_{k}\right\}$ and (i) if $\left(V_{i}, V_{j}\right)_{G^{\prime}}^{2}$ has density at least $d$ we place a heavy edge between $V_{i}$ and $V_{j}$ in $\Gamma$; (ii) if $\left(V_{i}, V_{j}\right)_{G^{\prime}}^{2}$ has density 0 and $\left(V_{i}, V_{j}\right)_{G^{\prime}}^{1}$ has density at least $d$ we place a light edge between $V_{i}$ and $V_{j}$ in $\Gamma$; (iii) otherwise $V_{i}$ and $V_{j}$ are not adjacent in $\Gamma$.

The next result implies that the minimum degree of a multigraph is almost inherited by its reduced multigraph.

Lemma 5.4. Let $\varepsilon>0, d \in[0,1], M^{\prime}, n \in \mathbb{N}$ and let $G$ be a multigraph on $n$ vertices. Let $G^{\prime}$ be the pure multigraph and $\Gamma$ be the reduced multigraph obtained by applying Lemma 5.3 to $G$ with parameters $\varepsilon, d$ and $M^{\prime}$. Then $\delta(\Gamma) \geqslant(\delta(G) / n-(8 d+6 \varepsilon))|\Gamma|$.

Proof. Note that for all $V_{i} \in V(\Gamma)$,

$$
d_{\Gamma}\left(V_{i}\right)=d_{\Gamma}^{1}\left(V_{i}\right)+2 d_{\Gamma}^{2}\left(V_{i}\right)=\left|N_{\Gamma}\left(V_{i}\right)\right|+d_{\Gamma}^{2}\left(V_{i}\right) .
$$

Let $v \in V_{i}$. Notice that $N_{G^{\prime}}(v)$ intersects at least $\left(\left|N_{G^{\prime}}(v)\right|-\left|V_{0}\right|\right) /\left|V_{1}\right|$ clusters and thus by Lemma 5.3,

$$
\left|N_{\Gamma}\left(V_{i}\right)\right| \geqslant\left(\left|N_{G^{\prime}}(v)\right|-\left|V_{0}\right|\right) /\left|V_{1}\right| \geqslant\left(d_{G}^{1}(v)+d_{G}^{2}(v)-(4 d+3 \varepsilon) n\right) /\left|V_{1}\right| .
$$

Also note that $N_{G^{\prime}}^{2}(v)$ intersects at least $\left(\left|N_{G^{\prime}}^{2}(v)\right|-\left|V_{0}\right|\right) /\left|V_{1}\right|$ clusters and thus by Lemma 5.3,

$$
d_{\Gamma}^{2}\left(V_{i}\right) \geqslant\left(\left|N_{G^{\prime}}^{2}(v)\right|-\left|V_{0}\right|\right) /\left|V_{1}\right| \geqslant\left(d_{G}^{2}(v)-(4 d+3 \varepsilon) n\right) /\left|V_{1}\right| .
$$


Altogether this gives

$$
\begin{aligned}
d_{\Gamma}\left(V_{i}\right)=\left|N_{\Gamma}\left(V_{i}\right)\right|+d_{\Gamma}^{2}\left(V_{i}\right) \geqslant & \left(d_{G}^{1}(v)+d_{G}^{2}(v)-(4 d+3 \varepsilon) n\right) /\left|V_{1}\right| \\
& +\left(d_{G}^{2}(v)-(4 d+3 \varepsilon) n\right) /\left|V_{1}\right| \\
= & \left(d_{G}^{1}(v)+2 d_{G}^{2}(v)-(8 d+6 \varepsilon) n\right) /\left|V_{1}\right| \\
\geqslant & (\delta(G) / n-(8 d+6 \varepsilon))|\Gamma| .
\end{aligned}
$$

Therefore, $\delta(\Gamma) \geqslant(\delta(G) / n-(8 d+6 \varepsilon))|\Gamma|$, as claimed.

\section{Almost tiling multigraphs with $\overline{\mathcal{K}}_{r}$}

In order to prove Theorem 1.8, we apply (a corollary of) the following result. Roughly speaking, it states that every standard multigraph with minimum degree slightly greater than that in Theorem 1.8 contains an almost perfect $\overline{\mathcal{K}}_{r}$-tiling.

THEOREM 6.1. Let $n, r \in \mathbb{N}$ where $r \geqslant 2$ and $\eta>0$ such that $0<1 / n \ll \eta \ll$ $1 / r$. Suppose that $G$ is a standard multigraph on $n$ vertices such that

$$
\delta(G) \geqslant 2(1-1 / r+\eta) n .
$$

Then $G$ contains a $\overline{\mathcal{K}}_{r}$-tiling covering all but at most $\eta n$ vertices.

The next result is the key tool in the proof of Theorem 6.1.

LEMMA 6.2. Let $\eta, \gamma>0$ and $n, r \geqslant 2$ be integers such that $0<1 / n \ll \gamma \ll$ $\eta \ll 1 / r$. Let $G$ be a standard multigraph on $n$ vertices so that

$$
\delta(G) \geqslant 2(1-1 / r+\eta) n .
$$

Further, suppose that the largest $\overline{\mathcal{K}}_{r}$-tiling in $G$ covers precisely $n^{\prime} \leqslant(1-\eta) n$ vertices. Then there exists a $\left(\overline{\mathcal{K}}_{r} \cup \overline{\mathcal{K}}_{r+1}\right)$-tiling in $G$ that covers at least $n^{\prime}+\gamma n$ vertices.

Proof. Certainly Theorem 4.2 and (2) imply that $n^{\prime} \geqslant \eta n$. Let $\mathcal{M}$ denote a $\overline{\mathcal{K}}_{r^{-}}$ tiling in $G$ containing precisely $n^{\prime}$ vertices so that the total number of edges in $\mathcal{M}$ is maximized. Set $n^{\prime \prime}:=n-n^{\prime}$ and $G^{\prime \prime}:=G \backslash V(\mathcal{M})$.

CLAIM 6.3. There are at least $\gamma n$ vertices $x \in V\left(G^{\prime \prime}\right)$ such that $d_{G}(x, V(\mathcal{M})) \geqslant$ $2(1-1 / r) n^{\prime}+2 \gamma n$.

Suppose for a contradiction the claim is false. Then by (2), at least $n^{\prime \prime}-\gamma n$ vertices $y \in V\left(G^{\prime \prime}\right)$ are such that $d_{G^{\prime \prime}}(y) \geqslant 2(1-1 / r+\eta) n-2(1-1 / r) n^{\prime}-2 \gamma n \geqslant 2(1-$ 
$1 / r+\eta / 2) n^{\prime \prime}$. Thus by Theorem $4.2, G^{\prime \prime}$ contains a copy of some $U \in \overline{\mathcal{K}}_{r}$. But then together with $\mathcal{M}$, this forms a $\overline{\mathcal{K}}_{r}$-tiling on $n^{\prime}+r$ vertices in $G$, a contradiction to the maximality of $\mathcal{M}$. This proves the claim.

Given any $x \in V\left(G^{\prime \prime}\right)$ such that $d_{G}(x, V(\mathcal{M})) \geqslant 2(1-1 / r) n^{\prime}+2 \gamma n$, there are at least $\gamma n$ elements $U$ in $\mathcal{M}$ so that $d_{G}(x, U) \geqslant 2 r-1$. If $d_{G}(x, U)=2 r$, then since $U \in \overline{\mathcal{K}}_{r}, V(U) \cup\{x\}$ spans a copy of an element of $\overline{\mathcal{K}}_{r+1}$ in $G$. Otherwise there is precisely one vertex $y \in V(U)$ such that $x y$ is a light edge.

Suppose that for some $z \in V(U) \backslash\{y\}, z y$ is a light edge in $G$. Then since there are all possible edges between $x$ and $V(U) \backslash\{y\},(V(U) \backslash\{y\}) \cup\{x\}$ spans a copy of some $U^{\prime} \in \overline{\mathcal{K}}_{r}$ such that $\left|E\left(U^{\prime}\right)\right|=|E(U)|+1$. This is a contradiction to the choice of $\mathcal{M}$. Thus for all $z \in V(U) \backslash\{y\}, y z$ is a heavy edge. This implies that $V(U) \cup\{x\}$ spans a copy of some $U^{\prime} \in \mathcal{\mathcal { K }}_{r+1}$.

Claim 6.3 implies there are at least $\gamma n$ vertices $x \in V\left(G^{\prime \prime}\right)$ such that $d_{G}(x$, $V(\mathcal{M})) \geqslant 2(1-1 / r) n^{\prime}+2 \gamma n$. So for at least $\gamma n$ such vertices $x$, we can pair them off with distinct elements $U$ of $\mathcal{M}$ so that $V(U) \cup\{x\}$ spans a copy of an element of $\overline{\mathcal{K}}_{r+1}$. This therefore implies that there exists a $\left(\overline{\mathcal{K}}_{r} \cup \overline{\mathcal{K}}_{r+1}\right)$-tiling in $G$ that covers at least $n^{\prime}+\gamma n$ vertices, as desired.

The next simple observation will be used in the proof of Theorem 6.1 to convert a $\left(\overline{\mathcal{K}}_{r} \cup \overline{\mathcal{K}}_{r+1}\right)$-tiling in the reduced multigraph $\Gamma$ of $G$ into a $\overline{\mathcal{K}}_{r}$-tiling in the blowup $\Gamma(r)$ of $\Gamma$.

FACT 6.4. Suppose that $r, t \in \mathbb{N}$ such that $r$ divides $t$. If $U \in\left(\overline{\mathcal{K}}_{r} \cup \overline{\mathcal{K}}_{r+1}\right)$ then $U(t)$ contains a perfect $\overline{\mathcal{K}}_{r}$-tiling.

We are now ready to prove Theorem 6.1. We repeatedly apply Lemma 6.2 and Fact 6.4 to obtain an almost perfect $\overline{\mathcal{K}}_{r}$-tiling in a blow-up of the reduced multigraph of $G$. Applying Lemma 5.2 will then yield an almost perfect $\overline{\mathcal{K}}_{r}$-tiling in $G$. Arguments of a similar nature were applied in $[8,16,28]$.

Proof of Theorem 6.1. Define additional constants $\varepsilon, d, \gamma$ and $M^{\prime} \in \mathbb{N}$ so that $0<1 / n \ll 1 / M^{\prime} \ll \varepsilon \ll d \ll \gamma \ll \eta \ll 1 / r$. Set $z:=\lceil 1 / \gamma\rceil$. Apply Lemma 5.3 with parameters $\varepsilon, d$ and $M^{\prime}$ to $G$ to obtain clusters $V_{1}, \ldots, V_{k}$, an exceptional set $V_{0}$ and a pure multigraph $G^{\prime}$. Set $m:=\left|V_{1}\right|=\cdots=\left|V_{k}\right|$. Let $\Gamma$ be the reduced multigraph of $G$ with parameters $\varepsilon, d$ and $M^{\prime}$. Lemma 5.4 implies that

$$
\delta(\Gamma) \geqslant 2(1-1 / r+\eta / 2) k
$$

Claim 6.5. $\Gamma^{\prime}:=\Gamma\left(r^{z}\right)$ contains a $\overline{\mathcal{K}}_{r}$-tiling covering at least $(1-\eta / 2) k r^{z}=$ $(1-\eta / 2)\left|\Gamma^{\prime}\right|$ vertices. 
If $\Gamma$ contains a $\overline{\mathcal{K}}_{r}$-tiling covering at least $(1-\eta / 2) k$ vertices then Fact 6.4 implies that Claim 6.5 holds. So suppose that the largest $\overline{\mathcal{K}}_{r}$-tiling in $\Gamma$ covers precisely $\ell \leqslant(1-\eta / 2) k$ vertices. Then by Lemma $6.2, \Gamma$ contains a $\left(\overline{\mathcal{K}}_{r} \cup \overline{\mathcal{K}}_{r+1}\right)$ tiling that covers at least $\ell+\gamma k$ vertices. Thus, by Fact 6.4, $\Gamma(r)$ contains a $\overline{\mathcal{K}}_{r}$-tiling covering at least $(\ell+\gamma k) r$ vertices. (So at least a $\gamma$-proportion of the vertices in $\Gamma(r)$ are covered.) Further, by definition of $\Gamma(r)$ and (3),

$$
\delta(\Gamma(r)) \geqslant 2(1-1 / r+\eta / 2) k r .
$$

If $\Gamma(r)$ contains a $\overline{\mathcal{K}}_{r}$-tiling covering at least $(1-\eta / 2) k r$ vertices then again Fact 6.4 implies that the claim holds. So suppose that the largest $\overline{\mathcal{K}}_{r}$-tiling in $\Gamma(r)$ covers precisely $\ell^{\prime} \leqslant(1-\eta / 2) k r$ vertices. Recall that $\ell^{\prime} \geqslant(\ell+\gamma k) r$. By Lemma $6.2, \Gamma(r)$ contains a $\left(\overline{\mathcal{K}}_{r} \cup \overline{\mathcal{K}}_{r+1}\right)$-tiling that covers at least $\ell^{\prime}+\gamma k r \geqslant$ $(\ell+2 \gamma k) r$ vertices. Thus, by Fact $6.4, \Gamma\left(r^{2}\right)$ contains a $\overline{\mathcal{K}}_{r}$-tiling covering at least $(\ell+2 \gamma k) r^{2}$ vertices. (So at least a $2 \gamma$-proportion of the vertices in $\Gamma\left(r^{2}\right)$ are covered.) Repeating this argument at most $z$ times we see that the claim holds.

For each $1 \leqslant i \leqslant k$, partition $V_{i}$ into classes $V_{i}^{*}, V_{i, 1}, \ldots, V_{i, r^{z}}$ where $m^{\prime}:=$ $\left|V_{i, j}\right|=\left\lfloor m / r^{z}\right\rfloor \geqslant m /\left(2 r^{z}\right)$ for all $1 \leqslant j \leqslant r^{z}$. Since $m k \geqslant(1-\varepsilon) n$ by Lemma 5.3,

$$
m^{\prime}\left|\Gamma^{\prime}\right|=\left\lfloor m / r^{z}\right\rfloor k r^{z} \geqslant m k-k r^{z} \geqslant(1-2 \varepsilon) n .
$$

Let $c \in$ [2]. Lemma 5.1 implies that if $\left(V_{i_{1}}, V_{i_{2}}\right)_{G^{\prime}}^{c}$ is $\varepsilon$-regular with density at least $d$ then $\left(V_{i_{1}, j_{1}}, V_{i_{2}, j_{2}}\right)_{G^{\prime}}^{c}$ is $2 \varepsilon r^{z}$-regular with density at least $d-\varepsilon \geqslant d / 2$ (for all $1 \leqslant j_{1}, j_{2} \leqslant r^{z}$ ). In particular, we can label the vertex set of $\Gamma^{\prime}$ so that $V\left(\Gamma^{\prime}\right)=\left\{V_{i, j}: 1 \leqslant i \leqslant k, 1 \leqslant j \leqslant r^{z}\right\}$ where, for $c \in[2], \mu\left(V_{i_{1}, j_{1}} V_{i_{2}, j_{2}}\right)=c$ in $\Gamma^{\prime}$ implies that $\left(V_{i_{1}, j_{1}}, V_{i_{2}, j_{2}}\right)_{G^{\prime}}^{c}$ is $2 \varepsilon r^{z}$-regular with density at least $d / 2$.

By Claim $6.5, \Gamma^{\prime}$ has a $\overline{\mathcal{K}}_{r}$-tiling $\mathcal{M}$ that contains at least $(1-\eta / 2)\left|\Gamma^{\prime}\right|$ vertices. Consider any element $U$ in $\mathcal{M}$ and let $V(U)=\left\{V_{i_{1}, j_{1}}, V_{i_{2}, j_{2}}, \ldots, V_{i_{r}, j_{r}}\right\}$. Set $V^{\prime}$ to be the union of $V_{i_{1}, j_{1}}, V_{i_{2}, j_{2}}, \ldots, V_{i_{r}, j_{r}}$. Note that $0<1 / m^{\prime} \ll 2 \varepsilon r^{z} \ll d / 2 \ll$ $\gamma \ll 1 / r$. Thus, Lemma 5.2 implies that $G^{\prime}\left[V^{\prime}\right]$ contains a $\overline{\mathcal{K}}_{r}$-tiling covering all but at most $\sqrt{2 \varepsilon r^{z}} m^{\prime} r \leqslant \gamma m^{\prime} r$ vertices. (Here we are using that a heavy edge in $\Gamma^{\prime}$ corresponds to a $2 \varepsilon r^{z}$-regular pair in $G^{\prime}$ consisting only of heavy edges, and a light edge in $\Gamma^{\prime}$ corresponds to a $2 \varepsilon r^{z}$-regular pair in $G^{\prime}$ consisting only of light edges.) By considering each element in $\mathcal{M}$ we conclude that $G^{\prime} \subseteq G$ contains a $\overline{\mathcal{K}}_{r}$-tiling covering at least

$$
(1-\gamma) m^{\prime} r \times(1-\eta / 2)\left|\Gamma^{\prime}\right| / r \stackrel{(4)}{\geqslant}(1-\gamma)(1-\eta / 2)(1-2 \varepsilon) n \geqslant(1-\eta) n
$$

vertices, as desired. 
The following result is a simple consequence of Theorem 6.1.

Corollary 6.6. Let $r \geqslant 2$ and $0<1 / n \ll \eta \ll 1 / r$. Suppose that $G$ is a multigraph on $n$ vertices such that

$$
\delta(G) \geqslant 2(1-1 / r-\eta) n .
$$

Then $G$ contains a $\overline{\mathcal{K}}_{r}$-tiling covering all but at most $4 r^{2} \eta n$ vertices.

Proof. Add $n^{\prime}:=\lceil 2 \eta n /(1 / r-\eta)\rceil$ vertices to $G$ which send out heavy edges to all other vertices (including each other). Call the resulting multigraph $G^{*}$. Since $\delta\left(G^{*}\right)=\delta(G)+2 n^{\prime}=\delta(G)+2(1-1 / r+\eta) n^{\prime}+2(1 / r-\eta) n^{\prime} \geqslant 2(1-1 / r+\eta)\left(n+n^{\prime}\right)$, we can apply Theorem 6.1 to $G^{*}$ to obtain a $\overline{\mathcal{K}}_{r}$-tiling in $G^{*}$ covering all but at most $\eta\left(n+n^{\prime}\right)$ vertices. Removing all those tiles that contain vertices from $V\left(G^{*}\right) \backslash V(G)$, we obtain a $\overline{\mathcal{K}}_{r}$-tiling in $G$ that covers all but at most $\eta\left(n+n^{\prime}\right)+$ $(r-1) n^{\prime} \leqslant 4 r^{2} \eta n$ of the vertices of $G$, as desired.

\section{Almost perfect tilings in the nonextremal case}

Suppose that, in the proof of Theorem 1.8 we have found a small absorbing set $M$. Ideally, we would next like to apply Corollary 6.6 to conclude that $G \backslash V(M)$ contains an almost perfect $\mathcal{U}_{r}$-tiling $\mathcal{M}$, and then use $M$ to cover the remaining vertices, thereby obtaining a perfect $\mathcal{U}_{r}$-tiling in $G$. However, to achieve this we would require that the set of vertices uncovered by $\mathcal{M}$ is much smaller than the size of the absorbing set $M$. Corollary 6.6 does not guarantee this though. Indeed, this is because the size of the set of uncovered vertices in Corollary 6.6 is large compared to the parameter $\eta$. Worst still, it is easy to see that the conclusion of Corollary 6.6 is false if we replace $4 r^{2} \eta n$ with a term significantly smaller than $\eta n$.

Therefore, instead we show that the conclusion of Corollary 6.6 can be strengthened in the desired way if our multigraph $G$ is far from extremal. (This strengthening will be at the cost of no longer guaranteeing an almost perfect $\overline{\mathcal{K}}_{r}$ tiling, but rather an almost perfect $\mathcal{K}_{r}^{\prime}$-tiling.) This will ensure that we can then use the above approach in the nonextremal case (we then have to deal with the extremal case separately).

To precisely describe the multigraphs that are far from extremal, we use the following definition.

Definition 7.1. Given $\gamma>0$ and $r \in \mathbb{N}$, we say that a multigraph $G$ on $n$ vertices is $(1 / r, \gamma)$-extremal if 
(i) there exists $S \subseteq V(G)$ such that ||$S|-n / r|<\gamma n$ and $e(G[S])<\gamma n^{2}$; or

(ii) there exists $S \subseteq V(G)$ such that ||$S|-2 n / r|<\gamma n$ and $e_{2}(G[S])<\gamma n^{2}$.

The aim of this section is to prove the following result.

THEOREM 7.2. Let $n, r \in \mathbb{N}$ where $r \geqslant 2$ and $\alpha, \eta, \gamma>0$ such that $0<1 / n \ll$ $\alpha \ll \eta \ll \gamma \ll 1 / r$ and let $G$ be a multigraph on $n$ vertices. If $G$ is not $(1 / r, \gamma)$ extremal and

$$
\delta(G) \geqslant 2(1-1 / r-\eta) n,
$$

then $G$ contains a $\mathcal{K}_{r}^{\prime}$-tiling covering all but at most $\alpha$ n vertices.

The proof of Theorem 7.2 makes use of Corollary 6.6. The next result will be used to convert an almost perfect tiling of a multigraph with universal graphs into a perfect tiling.

LEMMA 7.3. Let $n, r \in \mathbb{N}$ where $r \geqslant 2$ and $\tau, \gamma^{\prime}>0$ such that $0<1 / n \ll \tau \ll$ $\gamma^{\prime} \ll 1 / r$ and let $G$ be a multigraph on $n$ vertices. If $G$ is not $\left(1 / r, \gamma^{\prime}\right)$-extremal, $\delta(G) \geqslant 2(1-1 / r-\tau) n$, and there exists a $\overline{\mathcal{K}}_{r}$-tiling covering all but one vertex, then $G$ contains a perfect $\left(\mathcal{K}_{r}^{\prime} \cup \mathcal{K}_{r+1}^{\prime}\right)$-tiling in which all but at most three of the tiles are copies of $\overline{\mathcal{K}}_{r}$.

Proof. Let $\mathcal{T}=\left\{T_{1}, \ldots, T_{m}\right\}$ be the $\overline{\mathcal{K}}_{r}$-tiling in $G$ and let $v^{*}$ be the leftover vertex. If there exists $T \in \mathcal{T}$ such that $d\left(v^{*}, T\right) \geqslant 2 r-1$, then we obtain a perfect $\left(\mathcal{K}_{r}^{\prime} \cup \mathcal{K}_{r+1}^{\prime}\right)$-tiling in which all but one of the tiles are copies of $\overline{\mathcal{K}}_{r}$. So suppose that this is not the case. Then for all but at most

$$
(2 r-2)|\mathcal{T}|-\delta(G)=2(1-1 / r)(n-1)-\delta(G) \leqslant 3 \tau n
$$

of the $T \in \mathcal{T}, d\left(v^{*}, T\right)=2 r-2$.

If there exists $T \in \mathcal{T}$ such that $d\left(v^{*}, T\right)=2 r-2$, then we could move $v^{*}$ into $T$ to create a copy of $\mathcal{K}_{r+1}^{\prime}$ unless:

$(\alpha)$ there exists $u \in V(T)$ such that $\mu\left(v^{*} u\right)=0$ in $G$ or;

$(\beta) u_{1}, u_{2} \in V(T)$ are light neighbours of $v^{*}$ in $G$ and either

(a) $u_{1} u_{2}$ is a light edge or;

(b) $u_{1}$ and $u_{2}$ are incident with distinct light edges in $T$.

So we may suppose that one of $(\alpha)$ and $(\beta)$ holds whenever $d\left(v^{*}, T\right)=2 r-2$. 
Let

$$
B_{i}^{*}:=\left\{u \in T \in \mathcal{T}: d\left(v^{*}, T\right)=2 r-2 \text { and } \mu\left(v^{*} u\right)=i\right\} .
$$

Set $B^{*}:=B_{0}^{*} \cup B_{1}^{*}$. Let $\mathcal{T}^{*}$ to be the set of tiles in $\mathcal{T}$ which contain a vertex from $B^{*}$. Call an edge of $G$ useful if it either has both endpoints in $B_{0}^{*}$, or it has one endpoint in $B_{0}^{*}$ and the other in $B_{1}^{*}$, or it is a heavy edge with both endpoints in $B_{1}^{*}$.

Given distinct $T, T^{\prime} \in \mathcal{T}^{*}$, we say that the ordered pair $\left(T, T^{\prime}\right)$ is bad if

(i) there exists $b \in B^{*} \cap T$ such that $d\left(b, T^{\prime}\right) \neq 2 r-2$ or failing this;

(ii) there exists some $b^{\prime} \in B^{*} \cap T^{\prime}$ such that $d\left(b^{\prime}, T\right)=2 r-2$, and some $x \in T$ such that $b^{\prime} x$ is not a heavy edge and $d\left(x, T^{\prime}\right) \neq 2 r-2$.

If neither $\left(T, T^{\prime}\right)$ nor $\left(T^{\prime}, T\right)$ are bad, then we say that $\left\{T, T^{\prime}\right\}$ is good.

First we show that nonextremality guarantees that there is a useful edge $b b^{\prime}$ between a good pair $\left\{T, T^{\prime}\right\}$. Then we show how to use such a configuration to get the desired tiling which uses $v^{*}$.

Define an auxiliary digraph $\mathcal{D}$ with vertex set $\mathcal{T}^{*}$ where there is an edge from $T$ to $T^{\prime}$ if $\left(T, T^{\prime}\right)$ is bad.

Note that if there exists a $b \in B^{*}$ where $b \in T \in \mathcal{T}^{*}$ and a $T^{\prime} \in \mathcal{T} \backslash\{T\}$ such that $d\left(b, T^{\prime}\right) \geqslant 2 r-1$, then we obtain our desired perfect $\left(\mathcal{K}_{r}^{\prime} \cup \mathcal{K}_{r+1}^{\prime}\right)$-tiling by moving $b$ to $T^{\prime}$ to create a $\hat{\mathcal{K}}_{r+1}$ and then moving $v^{*}$ to $T$ to create a $\hat{\mathcal{K}}_{r}(b$ was a problem vertex for $v^{*}$; moving it out of $T$ means that we can move $v^{*}$ in). So we may assume that such a $b$ does not exist. Therefore, for all $b$ in $B^{*}$, by a computation similar to (5), we have that $d\left(b, T^{\prime}\right)=2 r-2$ for all but at most $3 \tau n$ of the $T^{\prime} \in \mathcal{T}$. In particular, for a fixed $T \in \mathcal{T}^{*}$ there are at most $6 \tau n T^{\prime} \in \mathcal{T}^{*}$ such that $\left(T, T^{\prime}\right)$ satisfies (i) in the definition of bad.

Given distinct $T, T^{\prime} \in \mathcal{T}^{*}$, suppose there exists $b^{\prime} \in B^{*} \cap T^{\prime}$ such that $d\left(b^{\prime}\right.$, $T)=2 r-2$, and some $x \in T$ such that $b^{\prime} x$ is not a heavy edge and $d\left(x, T^{\prime}\right) \geqslant$ $2 r-1$. By the previous paragraph, $x \notin B^{*}$. So $T \cup T^{\prime} \cup\left\{v^{*}\right\}$ spans two disjoint copies of $\hat{K}_{r}$ and $K_{r+1}^{\prime}$. (The vertex set of the former tile is $T-x+b^{\prime}$, the latter $T^{\prime}-b^{\prime}+x+v^{*}$.) In particular, we obtain our desired perfect $\left(\mathcal{K}_{r}^{\prime} \cup \mathcal{K}_{r+1}^{\prime}\right)$-tiling. So we may assume that such an $x$ does not exist.

Given distinct $T, T^{\prime} \in \mathcal{T}^{*}$, suppose there exists $b^{\prime} \in B^{*} \cap T^{\prime}$ such that $d\left(b^{\prime}\right.$, $T)=2 r-2$, and some $x \in T$ such that $b^{\prime} x$ is not a heavy edge. Further, suppose there exists $T^{\prime \prime} \in \mathcal{T} \backslash\left\{T, T^{\prime}\right\}$ such that $d\left(x, T^{\prime \prime}\right) \geqslant 2 r-1$. We can move $x$ to $T^{\prime \prime}$ to create a $\hat{\mathcal{K}}_{r+1}$, move $b^{\prime}$ to $T$ to create a $\hat{\mathcal{K}}_{r}$ and move $v^{*}$ to $T^{\prime}$ to create a $\hat{\mathcal{K}}_{r}$. In particular, we obtain our desired perfect $\left(\mathcal{K}_{r}^{\prime} \cup \mathcal{K}_{r+1}^{\prime}\right)$-tiling. Thus, we may assume that this is not the case.

Fix $T \in \mathcal{T}^{*}$. Suppose there are at least $3 r \tau n T^{\prime} \in \mathcal{T}^{*}$ such that $\left(T, T^{\prime}\right)$ satisfies (ii) in the definition of bad. Then there exists some vertex $w \in T$ that plays the 
role of $x$ in (ii) for at least $3 \tau n$ such $T^{\prime}$. But then the previous two paragraphs imply that

$$
d_{G}(w) \leqslant 3 \tau n(2 r-3)+(|\mathcal{T}|-3 \tau n)(2 r-2)+2<\delta(G),
$$

a contradiction.

Altogether this implies that $\mathcal{D}$ has maximum outdegree at most $6 r \tau n$ and so $e(\mathcal{D}) \leqslant 6 r \tau n^{2}$.

We now show that there are more than $6 r \tau n^{2}$ (unordered) pairs $\left\{T, T^{\prime}\right\}$ where $T, T^{\prime} \in \mathcal{T}^{*}$ and so that there is a useful edge in $G$ with one endpoint in $T$ and the other in $T^{\prime}$. Then for at least one such $\left\{T, T^{\prime}\right\}$ we have that neither $\left(T, T^{\prime}\right)$ nor $\left(T^{\prime}, T\right)$ is a bad pair.

By the nonextremality of $G$, if $\left|B_{0}^{*}\right| \geqslant\left(1-\gamma^{\prime}\right)(n / r)$ we have at least $\gamma^{\prime} n^{2} \gg$ $6 r \tau n^{2}$ useful edges in $G\left[B_{0}^{*}\right]$. If $\left|B^{*}\right| \geqslant\left(1-\gamma^{\prime}\right)(2 n / r)$, then we have at least $\gamma^{\prime} n^{2} \gg 6 r \tau n^{2}$ heavy edges in $G\left[B^{*}\right]$, all of which are useful (and at most $n$ of these edges go between vertices in the same tile from $\mathcal{T}^{*}$ ). Note that there are at most 4 useful edges between any $T$ and $T^{\prime}$ from $\mathcal{T}^{*}$. So we can assume that both $\left|B_{0}^{*}\right|<\left(1-\gamma^{\prime}\right)(n / r)$ and $\left|B^{*}\right|<\left(1-\gamma^{\prime}\right)(2 n / r)$. With the fact that $|\mathcal{T}|=(n-1) / r$, (5) implies that,

$$
\left|B^{*}\right|+\left|B_{0}^{*}\right|=2\left|B_{0}^{*}\right|+\left|B_{1}^{*}\right|=2\left|\mathcal{T}^{*}\right| \geqslant 2(|\mathcal{T}|-3 \tau n) \geqslant\left(1-\frac{\gamma^{\prime}}{4}\right) \frac{2 n}{r} .
$$

So $\left|B^{*}\right|<\left(1-\gamma^{\prime}\right)(2 n / r)$ implies that $\left|B_{0}^{*}\right|>\gamma^{\prime} n / r$, and $\left|B_{0}^{*}\right|<\left(1-\gamma^{\prime}\right)(n / r)$ implies that $\left|B^{*}\right|>\left(1+\gamma^{\prime} / 2\right)(n / r)$. Therefore, each of the at least $\gamma^{\prime} n / r$ vertices in $B_{0}^{*}$ is incident to at least $\left|B^{*}\right|+\delta(G) / 2-n \geqslant\left(\gamma^{\prime} n\right) /(4 r)$ useful edges. In total we have at least $(1 / 2) \times\left(\gamma^{\prime} n\right) /(4 r) \times\left(\gamma^{\prime} n\right) / r \gg 6 r \tau n^{2}$ useful edges in $G$ which ensures we find our desired pair $\left\{T, T^{\prime}\right\}$.

Now that we have a useful edge between a good pair, the next two claims show that this is sufficient to give us the desired perfect $\left(\mathcal{K}_{r}^{\prime} \cup \mathcal{K}_{r+1}^{\prime}\right)$-tiling.

Claim 7.4. Let $T, T^{\prime} \in \mathcal{T}^{*}$ be distinct and let $X \subseteq T$ and $X^{\prime} \subseteq T^{\prime}$ such that for all $x \in X, d\left(x, T^{\prime}\right)=2 r-2$ and for all $x^{\prime} \in X^{\prime}, d\left(x^{\prime}, T\right)=2 r-2$.

(i) If there exist $x \in X$ and $x^{\prime} \in X^{\prime}$ such that $\mu\left(x x^{\prime}\right)=0$, then $T-x+x^{\prime}$ and $T^{\prime}-x^{\prime}+x$ are both copies of $\overline{\mathcal{K}}_{r}$.

(ii) If there exist $x \in X$ and $x^{\prime} \in X^{\prime}$ such that $\mu\left(x x^{\prime}\right)=1$, then $T-x+x^{\prime}$ and $T^{\prime}-x^{\prime}+x$ are both copies of $\hat{\mathcal{K}}_{r}$.

(iii) If the bipartite graph of light edges induced by $X, X^{\prime}$ is 2-regular, then $T-$ $X+X^{\prime}$ and $T^{\prime}-X^{\prime}+X$ are both copies of $\overline{\mathcal{K}}_{r}$. 
The claim follows immediately if $\mu\left(x x^{\prime}\right)=0$. If $\mu\left(x x^{\prime}\right)=1$, then each vertex $x, x^{\prime}$ has one other light neighbour, each of which would create a $\hat{\mathcal{K}}_{r}$ after the switch. In the last case, all of the light neighbours of each vertex $x, x^{\prime}$ are being moved to the other side.

Claim 7.5. Suppose $T, T^{\prime} \in \mathcal{T}^{*}$ are distinct and there is a useful edge $b b^{\prime}$ where $b \in T, b^{\prime} \in T^{\prime}$ such that $d\left(b, T^{\prime}\right)=2 r-2$ and $d\left(b^{\prime}, T\right)=2 r-2$. Further suppose that for all $w \in T$, if $w b^{\prime}$ is not heavy, then $d\left(w, T^{\prime}\right)=2 r-2$ and for all $w^{\prime} \in T^{\prime}$ if $w^{\prime} b$ is not heavy, then $d\left(w^{\prime}, T\right)=2 r-2$. Then there is a $\left(\mathcal{K}_{r}^{\prime} \cup \mathcal{K}_{r+1}^{\prime}\right)$-tiling in $G$ covering precisely the vertices in $V(T) \cup V\left(T^{\prime}\right) \cup\left\{v^{*}\right\}$.

To prove the claim, we split the argument into three cases.

Case 1. There exists $w \in T \backslash\{b\}$ or $w^{\prime} \in T^{\prime} \backslash\left\{b^{\prime}\right\}$ such that either $\mu\left(b w^{\prime}\right)=0$ or $\mu\left(b^{\prime} w\right)=0$. Without loss of generality, suppose $\mu\left(b^{\prime} w\right)=0$. Switch $b^{\prime}$ and $w$. By Claim 7.4(i), $T-w+b^{\prime}$ and $T^{\prime}-b^{\prime}+w$ are $\overline{\mathcal{K}}_{r}$ s. Since $b b^{\prime}$ is a useful edge, $v^{*}$ sends at least $2 r-2$ edges to $T^{\prime}-b^{\prime}+w$. If $v^{*}$ sends at least $2 r-1$ edges to $T^{\prime}-b^{\prime}+w$ then $T^{\prime}-b^{\prime}+w+v^{*}$ is a copy of $\hat{\mathcal{K}}_{r+1}$. If $v^{*}$ sends precisely $2 r-2$ edges to $T^{\prime}-b^{\prime}+w$ then $w$ must be a light neighbour of $v^{*}$ in $G$. Further, as $\mu\left(b^{\prime} w\right)=0$ and $d\left(w, T^{\prime}\right)=2 r-2$, we have that $w$ sends all possible edges to $T^{\prime}-b^{\prime}+w$, that is $d\left(w, T^{\prime}-b^{\prime}+w\right)=2 r-2$. In particular, $(\alpha)$ and $(\beta)$ do not hold (where $T^{\prime}-b^{\prime}+w$ is playing the role of $T$ ). Thus, $T^{\prime}-b^{\prime}+w+v^{*}$ is a copy of $\mathcal{K}_{r+1}^{\prime}$.

Case 2. $b \in B_{0}^{*}$ or $b^{\prime} \in B_{0}^{*}$.

Without loss of generality, suppose $b \in B_{0}^{*}$. Since we are not in the first case, $b$ has two light neighbours in $T^{\prime}$. In particular, there exists $x^{\prime} \in V\left(T^{\prime}\right) \backslash B_{0}^{*}$ that is a light neighbour of $b$. Switch $b$ and $x^{\prime}$. By Claim 7.4(ii), $T-b+x^{\prime}$ and $T^{\prime}-x^{\prime}+b$ are copies of $\hat{\mathcal{K}}_{r}$. In particular, $T-b+x^{\prime}$ has the property that if $T-b+x^{\prime}$ contains a light path on 3 vertices, then $x^{\prime}$ is an endpoint of this path. Furthermore since $x^{\prime} \notin B_{0}^{*}$ and $\mu\left(v^{*} b\right)=0, v^{*}$ sends at least $2 r-1$ edges to $T-b+x^{\prime}$. Moreover, if $d\left(v^{*}, T-b+x^{\prime}\right)=2 r-1$, then $\mu\left(v^{*} x^{\prime}\right)=1$ and thus $T-b+x^{\prime}+v^{*}$ is a copy of $\mathcal{K}_{r+1}^{\prime}$.

Case 3: $b, b^{\prime} \in B_{1}^{*}$.

Let $B_{1}^{*} \cap T=\left\{b_{1}, b_{2}\right\}$ and $B_{1}^{*} \cap T^{\prime}=\left\{b_{1}^{\prime}, b_{2}^{\prime}\right\}$ with $b_{1}=b$ and $b_{1}^{\prime}=b^{\prime}$. By the definition of a useful edge, $\mu\left(b_{1} b_{1}^{\prime}\right)=2$. Since we are not in Case 1 , there exists $x^{\prime} \in V\left(T^{\prime}\right) \backslash B_{1}^{*}$ that is a light neighbour of $b_{1}$ and there exists $x \in V(T) \backslash B_{1}^{*}$ that is a light neighbour of $b_{1}^{\prime}$ (see Figure 3(i)). Since $T^{\prime}-x^{\prime}+b_{1} \in \hat{\mathcal{K}}_{r}$, we may assume that $T-b_{1}+x^{\prime}+v^{*} \notin \mathcal{K}_{r+1}^{\prime}$ - this implies that the other light neighbour of $x^{\prime}$ in $T$ must be $b_{2}$ and that $b_{2}$ must have a light neighbour in $T-b_{1}$. Similarly, we 

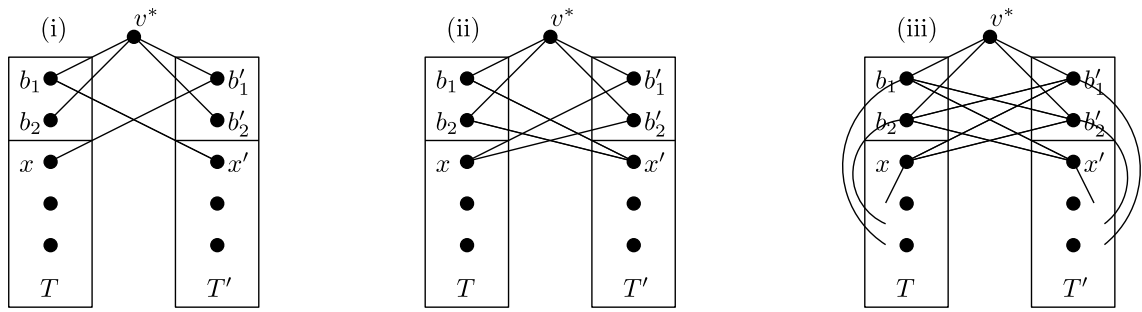

Figure 3. Case 3: Note that the light edges in $T$ and $T^{\prime}$ (not shown) form a matching.

can assume $T^{\prime}-b_{1}^{\prime}+x+v^{*} \notin \mathcal{K}_{r+1}^{\prime}$, so $x b_{2}^{\prime}$ is a light edge and $b_{2}^{\prime}$ has a (unique) light neighbour in $T^{\prime}-b_{1}^{\prime}$. Therefore, both $b_{1} b_{2}$ and $b_{1}^{\prime} b_{2}^{\prime}$ are heavy edges (see Figure 3(ii)).

Suppose $b_{1} b_{2}^{\prime}$ is not a light edge, so $b_{1}$ has a light neighbour $x_{2}^{\prime} \in V\left(T-x^{\prime}\right) \backslash B_{1}^{*}$. As in the previous case, since $T^{\prime}-x_{2}^{\prime}+b_{1} \in \hat{\mathcal{K}}_{r}$ we may assume that $T-b_{1}+x_{2}^{\prime}+$ $v^{*} \notin \mathcal{K}_{r+1}^{\prime}$. So it must be that $x_{2}^{\prime} b_{2}$ is a light edge. Now $b_{1} x^{\prime} b_{2} x_{2}^{\prime}$ forms a 4-cycle of light edges and by Claim 7.4(iii), we can switch $b_{1}, b_{2}$ for $x^{\prime}, x_{2}^{\prime}$ and then add $v^{*}$ to $T-b_{1}-b_{2}+x^{\prime}+x_{2}^{\prime}$ to obtain disjoint copies of $\overline{\mathcal{K}}_{r}$ and $\overrightarrow{\mathcal{K}}_{r+1}$. Likewise, we would be done if $b_{1}^{\prime} b_{2}$ is not a light edge. So suppose both $b_{1} b_{2}^{\prime}$ and $b_{1}^{\prime} b_{2}$ are light edges (see Figure 3(iii)). Then $b_{1} b_{2}^{\prime} x b_{1}^{\prime} b_{2} x^{\prime}$ forms a 6-cycle of light edges; we simultaneously switch $b_{1}, b_{2}, x$ for $b_{1}^{\prime}, b_{2}^{\prime}, x^{\prime}$ to obtain two disjoint copies of $\overline{\mathcal{K}}_{r}$. Recall $b_{1} b_{2}$ is a heavy edge so they both have distinct light neighbours in $T$. Hence, at most one of $b_{1}$ and $b_{2}$ is a light neighbour of $x$. Therefore, we can add $v^{*}$ to $T^{\prime}-b_{1}^{\prime}-b_{2}^{\prime}-x^{\prime}+b_{1}+b_{2}+x$ to form an element of $\mathcal{K}_{r+1}^{\prime}$. This completes the proof of the claim and thus the lemma.

We now combine Corollary 6.6 and Lemma 7.3 to obtain the following result.

Proposition 7.6. Let $n, r \in \mathbb{N}$ where $r \geqslant 2$ and $\eta, \gamma>0$ such that $0<1 / n \ll$ $\eta \ll \gamma \ll 1 / r$ and let $G$ be a multigraph on $n$ vertices. If $G$ is not $(1 / r, \gamma)$ extremal and

$$
\delta(G) \geqslant 2(1-1 / r-\eta) n,
$$

then $G$ contains a perfect $\left(\mathcal{K}_{r}^{\prime} \cup \mathcal{K}_{r+1}^{\prime}\right)$-tiling.

Proof. Choose $\tau, \gamma^{\prime}$ so that $\eta \ll \tau \ll \gamma^{\prime} \ll \gamma \ll 1 / r$. By Corollary 6.6 there exists a $\overline{\mathcal{K}}_{r}$-tiling $\mathcal{T}$ covering all but at most $4 r^{2} \eta n$ vertices. Set $U:=V(G) \backslash$ $V(\mathcal{T})$

To construct a perfect $\left(K_{r}^{\prime} \cup K_{r+1}^{\prime}\right)$-tiling in $G$, we perform the following iterative procedure. For each vertex $v^{*}$ uncovered by $\mathcal{T}$ we apply Lemma 7.3 once. 
In each iteration, we modify at most three elements of $\mathcal{T}$. Each time we apply Lemma 7.3, the multigraph under consideration is the subgraph of $G$ induced by $V\left(\mathcal{T}^{\prime}\right) \cup\left\{v^{*}\right\}$ where $\mathcal{T}^{\prime} \subseteq \mathcal{T}$ is the set of tiles in $\mathcal{T}$ that have not been modified in any of the previous steps.

Suppose we have performed this procedure for every vertex in some $U^{\prime} \subseteq U$. Let $G^{\prime}$ be the subgraph under consideration and note that $\left|G^{\prime}\right| \geqslant n-3 r\left|U^{\prime}\right|-$ $(|U|-1)$, so

$$
\delta\left(G^{\prime}\right) \geqslant 2(1-1 / r-\eta) n-\left(n-\left|G^{\prime}\right|\right) \geqslant 2(1-1 / r-\tau)\left|G^{\prime}\right| .
$$

Furthermore, if $S \subseteq V\left(G^{\prime}\right)$ and ||$S|-| G^{\prime}|/ r| \leqslant \gamma^{\prime}\left|G^{\prime}\right|$ or ||$S|-2| G^{\prime}|/ r| \leqslant \gamma^{\prime}\left|G^{\prime}\right|$, then ||$S|-n / r| \leqslant \gamma n$ or ||$S|-2 n / r| \leqslant \gamma n$, respectively. Therefore, $G^{\prime}$ is not $\left(1 / r, \gamma^{\prime}\right)$-extremal, because $\gamma n^{2} \geqslant \gamma^{\prime}\left|G^{\prime}\right|^{2}$. Hence, we may apply Lemma 7.3 a total of $|U|$ times to complete the proof.

We now apply the regularity lemma together with Proposition 7.6 to prove Theorem 7.2.

Proof of Theorem 7.2. Define additional constants $\varepsilon, d$ and $M^{\prime} \in \mathbb{N}$ so that $1 / n \ll$ $1 / M^{\prime} \ll \varepsilon \ll d \ll \alpha$. Apply Lemma 5.3 with parameters $\varepsilon, d$ and $M^{\prime}$ to $G$ to obtain clusters $V_{1}, \ldots, V_{k}$, an exceptional set $V_{0}$ and a pure multigraph $G^{\prime}$. Set $m:=\left|V_{1}\right|=\cdots=\left|V_{k}\right|$. Let $\Gamma$ be the reduced multigraph of $G$ with parameters $\varepsilon, d$ and $M^{\prime}$. Lemma 5.4 implies that

$$
\delta(\Gamma) \geqslant 2(1-1 / r-2 \eta) k .
$$

Suppose that there exists $S \subseteq V(\Gamma)$ such that ||$S|-k / r|<\gamma k / 4$ and $e(\Gamma[S])<\gamma k^{2} / 4$. Let $S=\left\{V_{i_{1}}, \ldots, V_{i_{t}}\right\}$ and $S^{\prime}:=V_{i_{1}} \cup \cdots \cup V_{i_{t}}$. Then ||$S^{\prime}|-k m / r|<\gamma k m / 4$ and so by Lemma 5.3(ii), ||$S^{\prime}|-n / r|<\gamma n$. Moreover, by Lemma 5.3(iv) and the definition of the reduced multigraph $\Gamma$, $e\left(G^{\prime}\left[S^{\prime}\right]\right)<\left(\gamma k^{2} / 4\right) \cdot 2 m^{2} \leqslant \gamma n^{2} / 2$. Thus, by Lemma 5.3(iii), $e\left(G\left[S^{\prime}\right]\right)<\gamma n^{2}$, a contradiction as $G$ is not $(1 / r, \gamma)$-extremal. A similar argument shows that there is no set $S \subseteq V(\Gamma)$ such that ||$S|-2 k / r|<\gamma k / 4$ and $e_{2}(\Gamma[S])<\gamma k^{2} / 4$. Thus, $\Gamma$ is not $(1 / r, \gamma / 4)$-extremal.

Therefore, by Proposition 7.6, $\Gamma$ contains a perfect $\left(\mathcal{K}_{r}^{\prime} \cup \mathcal{K}_{r+1}^{\prime}\right)$-tiling $\mathcal{T}$. Note that every induced subgraph of a copy of $\mathcal{K}_{r+1}^{\prime}$ of size $r$ is itself a copy of $\mathcal{K}_{r}^{\prime}$. Since $\varepsilon \ll d \ll \alpha$, by repeatedly applying Lemma 5.2 for each of the tiles in $\mathcal{T}$ we obtain a $\mathcal{K}_{r}^{\prime}$-tiling in $G$ covering all but at most $\alpha n$ vertices, as required.

\section{The absorbing lemma}

Let $G$ be a multigraph and $\mathcal{H}$ be a collection of multigraphs. We call a set $M \subseteq$ $V(G)$ an $\mathcal{H}$-absorbing set for $W \subseteq V(G)$ if both $G[M]$ and $G[M \cup W]$ contain 
perfect $\mathcal{H}$-tilings. Suppose that a nonextremal multigraph $G$ as in Theorem 1.8 contains a small set $M \subseteq V(G)$ that is a $\mathcal{K}_{r}^{\prime}$-absorbing set for any very small set $W \subseteq V(G)$. Theorem 7.2 ensures that $G \backslash M$ contains an almost perfect $\mathcal{K}_{r}^{\prime}$-tiling; let $W$ denote the set of uncovered vertices in $G \backslash M$. Then $G[M \cup W]$ contains a perfect $\mathcal{K}_{r}^{\prime}$-tiling, and thus $G$ contains a perfect $\mathcal{K}_{r}^{\prime}$-tiling, as required.

The next result gives a condition which forces a multigraph to contain an absorbing set.

Lemma 8.1 (Lo and Markström [22]). Let $h, t \in \mathbb{N}$ and let $\gamma>0$. Suppose that $\mathcal{H}$ is a collection of multigraphs, each on $h$ vertices. Then there exists an $n_{0} \in \mathbb{N}$ such that the following holds. Suppose that $G$ is a multigraph on $n \geqslant n_{0}$ vertices so that, for any $x, y \in V(G)$, there are at least $\gamma n^{\text {th-1 }}($ th -1$)$-sets $X \subseteq V(G)$ such that both $G[X \cup\{x\}]$ and $G[X \cup\{y\}]$ contain perfect $\mathcal{H}$-tilings. Then $V(G)$ contains a set $M$ so that

- $|M| \leqslant(\gamma / 2)^{h} n / 4$;

- $M$ is an $\mathcal{H}$-absorbing set for any $W \subseteq V(G) \backslash M$ such that $|W| \in h \mathbb{N}$ and $|W| \leqslant(\gamma / 2)^{2 h} h n / 32$.

Lo and Markström [22] proved Lemma 8.1 for hypergraphs, however, the proof of this result for multigraphs is identical.

We show that an absorbing set $M$ exists if $G$ is nonextremal and if additionally, in the case when $r=4, G$ is not 'splittable'. We use the following definition to make this precise.

DEFINITION 8.2. Given $\gamma>0$ and $r=2$, 4, a multigraph $G$ on $n$ vertices is $(r, \gamma)$-splittable if there exist disjoint sets $U_{1}, U_{2} \subseteq V(G)$ such that

- $\left|U_{1}\right|,\left|U_{2}\right| \geqslant(1 / 2-\gamma) n$ and;

- if $r=2$ then $e\left(U_{1}, U_{2}\right) \leqslant \gamma n^{2}$; if $r=4$ then $e_{2}\left(U_{1}, U_{2}\right) \leqslant \gamma n^{2}$.

To see why $r$ is included in Definition 8.2 as it is, note that if $\delta(G)$ is 'close' to $2(1-1 / 2) n$ and $G$ is $(2, \gamma)$-splittable, then $G$ is 'close' to the multigraph $M_{3}$ that was defined in the introduction with $r=2$ (see Figure 2). Similarly, if $\delta(G)$ is 'close' to $2(1-1 / 4) n$ and $G$ is $(4, \gamma)$-splittable, then $G$ is 'close' to the multigraph $M_{4}$ with $r=4$.

The next result (Lemma 2.1 restated) together with Lemma 8.1 implies our multigraph $G$ contains an absorbing set. 
LEMMA 8.3. Let $r \geqslant 3,0<1 / n \ll \eta, \phi \ll \gamma \ll 1 / r$, and let $G$ be a multigraph on $n$ vertices. If $\delta(G) \geqslant 2(1-1 / r-\eta) n$ and $G$ is not $(1 / r, \gamma)$-extremal and either $r \neq 4$ or $G$ is not $(4, \gamma)$-splittable, then for all distinct $x_{1}, x_{2} \in V(G)$ there exist at least $(\phi n)^{r-1}(r-1)$-sets $Y \subseteq V(G)$ such that $G\left[Y \cup\left\{x_{1}\right\}\right]$ and $G\left[Y \cup\left\{x_{2}\right\}\right]$ both contain $\mathcal{K}_{r}^{\prime}$.

We need the following lemma in the proof of Lemma 8.3.

LEMMA 8.4. Let $0<1 / n \ll \eta \ll \lambda \ll \gamma \ll 1$, and let $G$ be a multigraph on $n$ vertices with $\delta(G) \geqslant(3 / 2-2 \eta) n$ which is not $(1 / 4, \gamma)$-extremal and not $(4$, $\gamma)$-splittable. For any disjoint sets $U_{1}, U_{2} \subseteq V(G)$ such that $\left|U_{1}\right|,\left|U_{2}\right| \geqslant(1 / 2-$ $\gamma / 5) n$, there exists a collection $\mathcal{T}$ of copies of $\overline{\mathcal{K}}_{3}$ in $G\left[U_{1} \cup U_{2}\right]$ such that $|\mathcal{T}| \geqslant$ $\lambda n^{3}$ and for every $T \in \mathcal{T}$, both $V(T) \cap U_{1}$ and $V(T) \cap U_{2}$ are nonempty, and if $T$ contains a light edge $u_{1} u_{2}$, then $u_{1} \in U_{1}$ and $u_{2} \in U_{2}$.

Proof. For $T \in \overline{\mathcal{K}}_{3}$ such that $T \subseteq G\left[U_{1} \cup U_{2}\right]$, we say that $T$ is nice if both $V(T) \cap U_{1}$ and $V(T) \cap U_{2}$ are nonempty, and either $T$ has no light edges or $T$ has exactly one light edge $u_{1} u_{2}$ with $u_{1} \in U_{1}$ and $u_{2} \in U_{2}$. Since $G$ is not (4, $\gamma$ )-splittable, there exist at least $\gamma n^{2}$ heavy edges in $G$ with one endpoint in $U_{1}$ and one endpoint in $U_{2}$. For each such edge $u_{1} u_{2}$, we either find (i) at least $\gamma n / 2$ vertices $u$ such that $u_{1} u_{2} u$ is a nice $\overline{\mathcal{K}}_{3}$, or (ii) at least $\gamma n^{2}$ edges $e$ such that $u_{i} e$ is a nice $\overline{\mathcal{K}}_{3}$ for some $i \in[2]$. A simple calculation then implies that we obtain our desired collection of nice $\overline{\mathcal{K}}_{3}$.

Let $u_{1} u_{2}$ be an edge in $E_{2}\left(U_{1}, U_{2}\right)$ such that $u_{i} \in U_{i}$ for $i \in$ [2]. Pick $i$ so that $d^{2}\left(u_{i}\right) \geqslant d^{2}\left(u_{3-i}\right)$ and note that

$$
(3 / 2-2 \eta) n \leqslant\left|N^{2}\left(u_{3-i}\right)\right|+\left|N\left(u_{3-i}\right)\right| \leqslant\left|N^{2}\left(u_{i}\right)\right|+\left|N\left(u_{3-i}\right)\right|,
$$

so $\left|N^{2}\left(u_{i}\right) \cap N\left(u_{3-i}\right)\right| \geqslant n / 2-2 \eta n$. Since for every $u \in N^{2}\left(u_{i}\right) \cap N\left(u_{3-i}\right) \cap U_{i}$, $u_{1} u_{2} u$ is a nice $\overline{\mathcal{K}}_{3}$, if $\left|N^{2}\left(u_{i}\right) \cap N\left(u_{3-i}\right) \cap U_{i}\right| \geqslant \gamma n / 2$ we are done. Otherwise,

$$
\begin{aligned}
\left|N^{2}\left(u_{i}\right) \cap U_{3-i}\right| & \geqslant\left|N^{2}\left(u_{i}\right) \cap N\left(u_{3-i}\right) \cap\left(U_{1} \cup U_{2}\right)\right|-\left|N^{2}\left(u_{i}\right) \cap N\left(u_{3-i}\right) \cap U_{i}\right| \\
& \geqslant[n / 2-2 \eta n+2(1 / 2-\gamma / 5) n-n]-\gamma n / 2 \geqslant(1 / 2-\gamma) n .
\end{aligned}
$$

Therefore, since $G$ is not $(1 / 4, \gamma)$-extremal, there are at least $\gamma n^{2}$ heavy edges in $G\left[N^{2}\left(u_{i}\right) \cap U_{3-i}\right]$ and for each such edge $e, u_{i} e$ is a nice $\overline{\mathcal{K}}_{3}$.

We are now ready to prove Lemma 8.3.

Proof of Lemma 8.3. Define $\lambda$ so that $\eta, \phi \ll \lambda \ll \gamma$. Fix distinct vertices $x_{1}$, $x_{2} \in V(G)$ and let $X:=\left\{x_{1}, x_{2}\right\}$. For any $U \subseteq V(G)$ (with $0 \leqslant|U| \leqslant r$ ) and 
integer $i \geqslant 0$, let

$$
S_{i}(U):=\{v \in V(G) \backslash U: d(v, U) \geqslant 2|U|-i\} .
$$

Note that when $U=\emptyset$, we trivially have $S_{i}(U)=V(G)$. By the degree condition,

$$
\begin{aligned}
& (2 r-2)|U| n / r-2|U| \eta n-|U|(|U|-1) \leqslant e_{G}(U, V(G) \backslash U) \\
& \quad \leqslant\left|S_{0}(U)\right|+\left|S_{1}(U)\right|+(2|U|-2) n .
\end{aligned}
$$

Therefore,

$$
\left|S_{0}(U)\right|+\left|S_{1}(U)\right| \geqslant(2 r-2|U|) n / r-3|U| \eta n .
$$

So since $\left|S_{1}(U)\right| \leqslant n$ and $S_{0}(U) \subseteq S_{1}(U)$,

$$
\begin{aligned}
& \left|S_{0}(U)\right| \geqslant(r-2|U|) n / r-3|U| \eta n \quad \text { and } \\
& \left|S_{1}(U)\right| \geqslant(r-|U|) n / r-3|U| \eta n / 2 .
\end{aligned}
$$

Call an $(r-1)$-set $Y \subseteq V(G) \operatorname{good}$ if both $G\left[Y+x_{1}\right]$ and $G\left[Y+x_{2}\right]$ contain $\mathcal{K}_{r}^{\prime}$. For $t \geqslant 0$ and $0 \leqslant l \leqslant\lfloor t / 2\rfloor$, we say that a $t$-set $Y \subseteq S_{0}(X)$ is $l$-acceptable if $G[Y]$ has exactly $l$ light edges and either:

- $t=0$;

- $t>0$ and $G[Y] \in \overline{\mathcal{K}}_{t}$; or

- $t=r-3$ and $G[Y] \in \hat{\mathcal{K}}_{t}$.

If $Y$ is $l$-acceptable for some $0 \leqslant l \leqslant\lfloor t / 2\rfloor$, then we say that $Y$ is acceptable; note that if $Y$ is an acceptable $t$-set, then both $G\left[Y+x_{1}\right]$ and $G\left[Y+x_{2}\right]$ contain $\hat{\mathcal{K}}_{t+1}$. For any acceptable $t$-set $Y$, let $S_{1}^{*}(Y)$ be the set of vertices $v \in S_{1}(Y) \cap S_{0}(X)$ such that if $y$ is the unique light neighbour of $v$ in $Y$, then $y$ is incident to a light edge in $Y$. Note that if $Y$ is an $l$-acceptable $t$-set, $v \in S_{1}^{*}(Y)$ and $y$ is unique light neighbour of $v$ in $Y$, then $Y^{\prime}:=Y-y+v$, is either $(l-1)$-acceptable or $(l-2)$-acceptable depending on whether $y$ is incident to one or two light edges in $Y$. Recall that $y$ can only be incident to two light edges in $Y$ if $t=r-3$ and $y$ is the middle vertex of a path on three vertices in $Y$ that consists of light edges.

For $0 \leqslant t \leqslant r-3$ and $0 \leqslant l \leqslant\lfloor t / 2\rfloor$, say that an $l$-acceptable $t$-set $Y$ is $\lambda$-extendible if at least one of the following four conditions holds:

(i) $t=0, r$ is even and $\left|S_{0}(X)\right| \geqslant \lambda n$;

(ii) $t=0, r=4$, and there are at least $(\lambda n)^{3} 3$-sets $Z$ such that $Z$ is a good 3-set; 
(iii) $(r-1)-t$ is even, and there are at least $(\lambda n)^{2} 2$-sets $Z$ such that $Y \cup Z$ is either an acceptable $(t+2)$-set or a good $(r-1)$-set; or

(iv) $\left|S_{1}^{*}(Y)\right| \geqslant \lambda n$.

If we assume that (iv) never holds, then Claim 8.5 below will imply that there exists at least $(\phi n)^{r-1}$ good $(r-1)$-sets. Indeed, in this case

- if $r$ is odd, then we can build good $(r-1)$-sets two vertices at a time by repeatedly using (iii);

- if $r$ is even and (i) holds, then we can construct good $(r-1)$-sets by first selecting any of the $\lambda n$ vertices in $S_{0}(X)$ and then finish the construction by repeatedly applying (iii); and

- if $r$ is even and (i) does not hold, then (ii) must hold which immediately implies that there are $(\lambda n)^{r-1} \operatorname{good}(r-1)$-sets.

ClAim 8.5. Let $0 \leqslant t \leqslant r-3$ and $0 \leqslant l \leqslant\lfloor t / 2\rfloor$, and $Y$ be an $l$-acceptable $t$-set. If $t=0$ or $(r-1)-t$ is even, then $Y$ is $\lambda$-extendible.

To prove the claim, we may assume that (iv) does not hold throughout, that is

$$
\left|S_{1}^{*}(Y)\right|<\lambda n
$$

Note that, by (8),

$$
\left|S_{0}(X)\right| \geqslant(r-4) n / r-6 \eta n .
$$

First assume that $(r-1)-t$ is even and let

$$
U:=S_{0}(X) \cap S_{0}(Y)=S_{0}(X \cup Y) .
$$

Also define

$$
\begin{array}{ll}
U^{\prime}:=\left(S_{0}(X) \cap S_{1}(Y)\right) \backslash S_{1}^{*}(Y) & \text { when } t \leqslant r-5, \quad \text { or } \\
U^{\prime}:=S_{1}(X \cup Y) \backslash S_{1}^{*}(Y) & \text { when } t=r-3 .
\end{array}
$$

When $t \leqslant r-5,(7),(10)$ and (11) imply that

$$
\begin{aligned}
|U|+\left|U^{\prime}\right| & \geqslant\left(\left|S_{0}(Y)\right|+\left|S_{0}(X)\right|-n\right)+\left(\left|S_{1}(Y)\right|-\left|S_{1}^{*}(Y)\right|+\left|S_{0}(X)\right|-n\right) \\
& \geqslant 2(r-t-4) n / r-2 \lambda n .
\end{aligned}
$$

Assume that $t \leqslant r-7$. If $|U| \geqslant \gamma n / 2$, then first pick any $z \in U$, and then pick any vertex $z^{\prime} \in N^{2}(z) \cap U^{\prime}$ and note that $Y+z+z^{\prime}$ is an acceptable $(t+2)$-set. 
By (12), the minimum degree condition and the fact that $U \subseteq U^{\prime}$, we have that there are at least

$$
3 n / r-\lambda n-(2 / r+2 \eta) n \geqslant n / r-2 \lambda n
$$

choices for $z^{\prime}$. Note that there are at least $(\gamma n / 2) \times(n / r-2 \lambda n) \times(1 / 2)>(\lambda n)^{2}$ choices for $\left\{z, z^{\prime}\right\}$, so (iii) in the definition of $\lambda$-extendible holds, as required. If $|U|<\gamma n / 2$, then pick any $z \in U^{\prime} \backslash U$ and let $y \in Y$ be the unique light neighbour of $z$ in $Y$. Recall that since $z \notin S_{1}^{*}(Y), y$ has no light neighbours in $Y$. By (12), $\left|U^{\prime} \backslash U\right| \geqslant 6 n / r-3 \lambda n$ and for any of the at least $2 n / r-2 \gamma n$ vertices $z^{\prime} \in$ $N^{2}(z) \cap N^{2}(y) \cap U^{\prime}, Y+z+z^{\prime}$ is an acceptable $(t+2)$-set. Note that there are at least $(6 n / r-3 \lambda n) \times(2 n / r-2 \gamma n) \times(1 / 2)>(\lambda n)^{2}$ choices for $\left\{z, z^{\prime}\right\}$, so (iii) in the definition of $\lambda$-extendible holds, as required.

When $t=r-5$, (12) implies that

$$
|U|+\left|U^{\prime}\right| \geqslant 2 n / r-2 \lambda n,
$$

and when $t=r-3,(7)$ and (10) give that

$$
|U|+\left|U^{\prime}\right| \geqslant\left|S_{0}(X \cup Y)\right|+\left|S_{1}(X \cup Y)\right|-\left|S_{1}^{*}(Y)\right| \geqslant 2 n / r-3(r-1) \eta n-\lambda n .
$$

Therefore, when $t \in\{r-3, r-5\}$,

$$
|U|+\left|U^{\prime}\right| \geqslant 2 n / r-\gamma n / 3 .
$$

We either find at least $\lambda n^{2}$ light edges $z z^{\prime}$ where $z \in U$ and $z^{\prime} \in U^{\prime}$, or at least $\lambda n^{2}$ heavy edges in $G\left[U^{\prime}\right]$. Note that, in either of these two cases, when $|Y|=r-5$, $G\left[Y+z+z^{\prime}+x_{i}\right] \in \hat{\mathcal{K}}_{r-2}$ for $i \in[2]$, and when $|Y|=r-3, G\left[Y+z+z^{\prime}+x_{i}\right]$ $\in \mathcal{K}_{r}^{\prime}$ for $i \in$ [2], so this will prove the claim. Suppose that we cannot find at least $\lambda n^{2}$ such edges. By nonextremality, this implies that $|U|<(1 / r-\gamma) n$ and $\left|U^{\prime}\right|<(2 / r-\gamma) n$. Hence, by (13), $|U| \geqslant 2 \gamma n / 3$ and $\left|U^{\prime}\right| \geqslant\left(1 / r+\frac{2}{3} \gamma\right) n$. Therefore, by the degree condition, any vertex in $z \in U$, is adjacent to at least $\gamma n / 2$ vertices $z^{\prime} \in U^{\prime}$, a contradiction.

We now show that if $r$ is even and $t=0$ and (i) in the definition of $\lambda$-extendible does not hold, then (ii) must hold. So assume $t=0, r$ is even and $\left|S_{0}(X)\right|<\lambda n$. This, with (8), implies that $r=4$ so $G$ is not $(r, \gamma)$-splittable. By the degree condition, $\left|S_{0}(X)\right|<\lambda n$ implies that

$2 n / r-2 \eta n \leqslant\left|N^{2}\left(x_{i}\right)\right| \leqslant 2 n / r+2 \lambda n \quad$ and $\quad\left|N\left(x_{i}\right)\right| \geqslant(1-3 \lambda) n$ for $i \in[2]$.

Therefore, if we let

$$
U_{i}:=\left(N^{2}\left(x_{i}\right) \cap N\left(x_{3-i}\right)\right) \backslash N^{2}\left(x_{3-i}\right) \text { for } i \in[2],
$$


then $U_{1}$ and $U_{2}$ are disjoint, and

$$
\left|U_{i}\right| \geqslant\left(\left|N^{2}\left(x_{i}\right)\right|+\left|N\left(x_{3-i}\right)\right|-n\right)-\left|S_{0}(X)\right| \geqslant(2 / r-5 \lambda) n \quad \text { for } i \in[2] .
$$

By Lemma 8.4, there are at least $\lambda n^{3}$ nice copies of $\overline{\mathcal{K}}_{3}$ in $G\left[U_{1} \cup U_{2}\right]$ (that is, copies of $\overline{\mathcal{K}}_{3}$ that intersect both $U_{1}$ and $U_{2}$ and that have at most one light edge and such a light edge has one endpoint in $U_{1}$ and the other in $U_{2}$ ). The vertex set of any such nice $\overline{\mathcal{K}}_{3}$ is a good $(r-1)$-set, as desired. This completes the proof of the claim.

Assume for a contradiction that there are at most $(\phi n)^{r-1} \operatorname{good}(r-1)$-sets. Let $0 \leqslant t \leqslant r-3$ be the maximum $t$ for which we have $(\phi n)^{t}$ acceptable $t$-sets and either $t=0$ or $(r-1)-t$ is even. Such a $t$ exists, since $\emptyset$ is an acceptable 0 -set. We can also assume that $t>0$, since if $Y=\emptyset$, then $S_{1}^{*}(Y)=\emptyset$ and (iv) cannot hold, so Claim 8.5 implies that one of (i), (ii) or (iii) must hold, which violates the maximality of $t$ or the assumption that there are at most $(\phi n)^{r-1}$ good $(r-1)$-sets. Let $l$ be minimal such that if $\mathcal{Y}$ is the set of $l$-acceptable $t$-sets, then $|\mathcal{Y}| \geqslant \lambda(\lambda / 16)^{\lfloor t / 2\rfloor-l}(\phi n)^{t}$. There exists such an $l$, because $\lambda \ll 1 / r$. By Claim 8.5 , each set $Y \in \mathcal{Y}$ is $\lambda$-extendible in one of two ways, so in particular there is some subset of $\mathcal{Y}$ of order at least $|\mathcal{Y}| / 2$ for which all elements are extendible in the same way, either (iii), or (iv). If the elements in this subset are all $\lambda$-extendible by (iii), then, because $\phi \ll \lambda \ll 1 / r$, we have at least

$$
\frac{(\lambda n)^{2} \cdot|\mathcal{Y}| / 2}{\left(\begin{array}{c}
t+2 \\
2
\end{array}\right)} \geqslant \frac{\lambda^{2} / 2 \cdot \lambda(\lambda / 16)^{\lfloor t / 2\rfloor}}{\left(\begin{array}{c}
t+2 \\
2
\end{array}\right)} \cdot \phi^{-2} \cdot(\phi n)^{t+2}>(\phi n)^{t+2}
$$

acceptable $(t+2)$-sets or, if $t=r-3$, more than $(\phi n)^{r-1} \operatorname{good}(r-1)$-sets. This contradicts the maximality of $t$ or the assumption that there are at most $(\phi n)^{r-1}$ good $(r-1)$-sets. (We divide by $\left(\begin{array}{c}t+2 \\ 2\end{array}\right)$ in the above calculation to account for the fact there are $\left(\begin{array}{c}t+2 \\ 2\end{array}\right)$ different ways a $(t+2)$-set can be constructed by adding 2 vertices to a $t$-set.)

Now assume that there are at least $|\mathcal{Y}| / 2 l$-acceptable $t$-sets that are extendible by (iv). Let $\mathcal{Y}^{\prime}$ be the collection of $(l-1)$-acceptable and $(l-2)$-acceptable $t$-sets and set

$$
\mathcal{Z}:=\left\{Y+z: Y \in \mathcal{Y} \text { and } z \in S_{1}^{*}(Y)\right\} .
$$

Our aim is to find a lower bound on $\left|\mathcal{Y}^{\prime}\right|$ which contradicts the minimality of $l$.

Let $Z \in \mathcal{Z}$ and let $Y \in \mathcal{Y}$ and $z \in S_{1}^{*}(Y)$ such that $Y+z=Z$. Note that the choice of $Y$ is not necessarily unique. Let $z^{\prime}$ be the unique light neighbour of $z$ in $Y$ and note that $Y^{\prime}=Y-z^{\prime}+z$ is in $\mathcal{Y}^{\prime}$. Therefore, for every $Z \in \mathcal{Z}$ there exists $Y^{\prime} \in \mathcal{Y}^{\prime}$ and $z^{\prime} \in V(G)$, such that $Z=Y^{\prime}+z^{\prime}$ which implies that $\left|\mathcal{Y}^{\prime}\right| \cdot n \geqslant|\mathcal{Z}|$.

Any $Z \in \mathcal{Z}$ is constructed by adding to some $Y \in \hat{\mathcal{K}}_{t}$ a vertex $z \in S_{1}^{*}(Y)$. So in any $Z \in \mathcal{Z}$ there are most four vertices $z^{\prime} \in Z$ such that there exists $z^{\prime \prime} \in Z$ such 
that $z^{\prime} z^{\prime \prime}$ is a light edge and $z^{\prime \prime}$ has at least two light neighbours in $Z$. Therefore, for every $Z \in \mathcal{Z}$, there are at most four different pairs $(Y, z)$ such that $Y \in \mathcal{Y}$, $z \in S_{1}^{*}(Y)$ and $Z=Y+z$. This implies that

$$
|\mathcal{Z}| \geqslant \frac{|\mathcal{Y}| / 2 \cdot \lambda n}{4} \geqslant(\lambda / 8) \cdot \lambda(\lambda / 16)^{\lfloor t / 2\rfloor-l}(\phi n)^{t} \cdot n
$$

so $\left|\mathcal{Y}^{\prime}\right| \geqslant(\lambda / 8) \cdot \lambda(\lambda / 16)^{\lfloor t / 2\rfloor-l}(\phi n)^{t}$. Therefore, there are at least

$$
\lambda(\lambda / 16)^{\lfloor t / 2\rfloor-(l-1)}(\phi n)^{t}
$$

$(l-1)$-acceptable $t$-sets or $(l-2)$-acceptable $t$-sets, a contradiction to the minimality of $l$.

\section{The stability result}

We now combine Lemmas 8.1 and 8.3 together with Theorem 7.2 to prove the following result which ensures that Theorem 1.8 holds in the case when $G$ is nonextremal and additionally if $r=4$, nonsplittable.

THEOREM 9.1. Let $n, r \in \mathbb{N}$ where $r$ divides $n$ and define $\eta, \gamma>0$ such that $0<1 / n \ll \eta \ll \gamma \ll 1 / r$. Let $G$ be a multigraph on $n$ vertices. If $\delta(G) \geqslant$ $2(1-1 / r-\eta) n$ and $G$ is not $(1 / r, \gamma)$-extremal and either $r \notin\{2,4\}$ or $G$ is not $(r, \gamma)$-splittable, then $G$ contains a perfect $\mathcal{K}_{r}^{\prime}$-tiling.

Proof. First assume that $r \geqslant 3$. Define $\alpha, \phi, \eta^{\prime}, \gamma^{\prime}>0$ so that $0<1 / n \ll \alpha \ll$ $\phi \ll \eta \ll \eta^{\prime} \ll \gamma^{\prime} \ll \gamma$. Let $G$ be as in the statement of the theorem. By Lemma 8.3, given any $x_{1}, x_{2} \in V(G)$, there exist at least $(\phi n)^{r-1}(r-1)$-sets $Y \subseteq V(G)$ such that both $G\left[Y \cup\left\{x_{1}\right\}\right]$ and $G\left[Y \cup\left\{x_{2}\right\}\right]$ contain elements of $\mathcal{K}_{r}^{\prime}$. Thus, by Lemma 8.1, $V(G)$ contains a set $M$ so that

- $|M| \leqslant\left((\phi)^{r-1} / 2\right)^{r} n / 4$;

- $M$ is a $\mathcal{K}_{r}^{\prime}$-absorbing set for any $W \subseteq V(G) \backslash M$ such that $|W| \in r \mathbb{N}$ and $|W| \leqslant\left((\phi)^{r-1} / 2\right)^{2 r} r n / 32$.

Let $G^{\prime}:=G \backslash V(M)$ and $n^{\prime}:=\left|G^{\prime}\right|$. So as $\phi \ll \eta \ll \eta^{\prime}$,

$$
\delta\left(G^{\prime}\right) \geqslant 2\left(1-1 / r-\eta^{\prime}\right) n^{\prime} .
$$

Further, as $\phi \ll \gamma^{\prime} \ll \gamma, G^{\prime}$ is not $\left(1 / r, \gamma^{\prime}\right)$-extremal. Thus, by Theorem 7.2, $G^{\prime}$ contains a $\mathcal{K}_{r}^{\prime}$-tiling covering all but at most $\alpha n^{\prime}<\left((\phi)^{r-1} / 2\right)^{2 r} r n / 32$ vertices. Let $W$ denote the set of these uncovered vertices. Then by definition of $M, G[M \cup W]$ 
contains a perfect $\mathcal{K}_{r}^{\prime}$-tiling. Altogether this implies that $G$ contains a perfect $\mathcal{K}_{r}^{\prime}$ tiling, as desired.

Now assume that $r \leqslant 2$. If $r=1$ the theorem is trivial. For $r=2$, we show that there exists a perfect matching in $H$, the graph underlying the multigraph $G$, that is $H$ is the graph on $V(G)$ in which there is an edge between $x$ and $y$ if and only if there is either a light or heavy edge between $x$ and $y$ in $G$. We have that $n$ is even, $\eta \ll \gamma \ll 1 / 2, \delta(H) \geqslant(1 / 2-\eta) n, G$ is not $(1 / 2, \gamma)$ extremal and $G$ is not $(2, \gamma)$-splittable. Let $M$ be a maximum matching in $H$ and suppose $M$ is not perfect. Note that the vertices unsaturated by $M$ form an independent set. Let $w_{1}, w_{2}$ be two vertices unsaturated by $M$ and for $i \in$ [2], define $S_{i}:=\left\{v: u v \in M\right.$ and $\left.u \in N\left(w_{i}\right)\right\}$. Note that $\left|S_{1}\right|,\left|S_{2}\right| \geqslant(1 / 2-\eta) n$ and there are no edges in $H$ with one endpoint in $S_{1}$ and the other in $S_{2}$ as this would give us an $M$-augmenting path, contradicting the maximality of $M$. Since $G$ is not $(2, \gamma)$-splittable, it cannot be the case that $S_{1}$ and $S_{2}$ are disjoint, so let $v \in S_{1} \cap S_{2}$. We have $N(v) \cap\left(S_{1} \cup S_{2}\right)=\emptyset$ and thus $\left|S_{1} \cup S_{2}\right| \leqslant(1 / 2+\eta) n$ which implies $\left|S_{1} \cap S_{2}\right| \geqslant(1 / 2-3 \eta) n \geqslant(1 / 2-\gamma) n$, contradicting the fact that $G$ is not $(1 / 2, \gamma)$-extremal.

\section{The extremal case}

In this section we prove the following theorem.

THEOREM 10.1. For any $r \in \mathbb{N}$, there exists $n_{0} \in \mathbb{N}$ such that the following holds. If $G$ is a multigraph on $n \geqslant n_{0}$ vertices, $n$ is divisible by $r$ and

$$
\delta(G) \geqslant 2(1-1 / r) n-1,
$$

then $G$ contains a perfect $\mathcal{K}_{r}^{\prime}$-tiling.

Note that Theorem 10.1 immediately implies Theorem 1.8 (and thus Theorem 1.4). Our results from the previous sections will ensure Theorem 10.1 holds in the 'nonextremal' cases. Therefore, most of the work in this section concerns the extremal cases.

Throughout this section we consider a standard multigraph $G$ on $n$ vertices that satisfies the hypothesis of Theorem 10.1. In particular, we may assume $1 / n \ll$ $1 / r$. We denote the vertex set of $G$ by $V$.

10.1. Preliminary claims. We use the following well-known and simple lemma in this section. A proof is included for completeness. 
LEMMA 10.2. For any graph $H$ there is a matching of order at least $\min \{\lfloor|H| / 2\rfloor, \delta(H)\}$.

Proof. Let $M$ be a maximal matching in $H$ and suppose that $|M|<\min \{\lfloor|H| / 2\rfloor$, $\delta(H)\}$. Let $U$ be the vertices incident to an edge in $M$ and let $W:=\bar{U}$. Note that $|W| \geqslant 2$ and that, by the maximality of $|M|, W$ is an independent set. Therefore, there exist distinct $x, y \in W$ where $d(x, U)+d(y, U) \geqslant 2 \delta(H)>2|M|$. Hence, there exists $e \in M$ such that $d(x, e)+d(y, e) \geqslant 3$, and this implies that there exists a matching of order $|M|+1$ in $H$.

The next claim gives us a minimum degree condition for $G[U]$ where $U$ is any set of size close to $s n / r$ for some $s \in \mathbb{N}$.

Claim 10.3. Suppose $0<1 / n \ll c \ll 1 / r$ and $s \in \mathbb{N}$ where $1 \leqslant s \leqslant r$. Let $v \in V$ and $U \subseteq V$. If

$$
s n / r-c n \leqslant|U| \leqslant s n / r+c n,
$$

then

$$
d(v, U) \geqslant 2(1-1 / s-r c / s)|U|
$$

Proof. When $s=1$ the statement is trivially true, so assume $s \geqslant 2$. Therefore,

$$
(1 / s+r c / s)|U| \geqslant(1 / s+r c / s)(s / r-c) n=\left(1 / r+(s-1) c / s-r c^{2} / s\right) n>n / r+1 .
$$

Hence, by (14),

$$
d(v, U) \geqslant \delta(G)-2|\bar{U}| \geqslant 2(|U|-(n / r+1))>2(1-1 / s-r c / s)|U| .
$$

Let $c$ be a constant such that $0<c<1$. We call a set $U \subseteq V$,

- a $(1, c)$-independent set if $|U|=n / r$ and $e(G[U])<c n^{2}$; or

- a $(2, c)$-independent set if $|U|=2 n / r$ and $e_{2}(G[U])<c n^{2}$.

If $U, U^{\prime} \subseteq V$ are vertex-disjoint, we say that the pair $\left\{U, U^{\prime}\right\}$ is

- a (1, c)-disconnected pair if $|U|=\left|U^{\prime}\right|=n / r$ and $e\left(U, U^{\prime}\right)<c n^{2}$; or

- a $(2, c)$-disconnected pair if $|U|=\left|U^{\prime}\right|=2 n / r$ and $e_{2}\left(U, U^{\prime}\right)<c n^{2}$.

If $1 \leqslant s \leqslant r$ and $U \subseteq V$, we call $U$ an $(s, c)$-tolerant set when $|U|=s n / r$ and

- for $t \in\{1,2\}, U$ does not contain a $(t, c)$-independent set; and

- if $s \in\{2,4\}$, then $U$ does not contain an $(s / 2, c)$-disconnected pair. 
The preceding definitions are closely related to the notion of being $(1 / r, \gamma)$ extremal (Definition 7.1) or $(r, \gamma)$-splittable (Definition 8.2) which the following simple claim makes explicit.

CLAIM 10.4. Let $1 \leqslant s \leqslant r$ where $s \in \mathbb{N}, 0<1 / n \ll c^{\prime} \ll \gamma \ll c \ll 1 / r$, $U \subseteq V$ such that $|U|=s n / r$ and $U^{\prime} \subseteq U$ such that $\left|U \triangle U^{\prime}\right| \leqslant c^{\prime} n$. If $U \subseteq V$ is $(s, c)$-tolerant, then $G\left[U^{\prime}\right]$ is not $(1 / s, \gamma)$-extremal and, when $s \in\{2,4\}, G\left[U^{\prime}\right]$ is not $(s, \gamma)$-splittable.

Proof. Suppose that $G\left[U^{\prime}\right]$ is $(1 / s, \gamma)$-extremal. So there exists $W \subseteq U^{\prime}$ such that either $e(G[W])<\gamma\left|U^{\prime}\right|^{2}$, and $|W| \geqslant(1 / s-\gamma)\left|U^{\prime}\right| \geqslant(1 / r-2 \gamma) n$, or $e_{2}(G[W])<\gamma\left|U^{\prime}\right|^{2}$, and $|W| \geqslant(2 / s-\gamma)\left|U^{\prime}\right| \geqslant(2 / r-2 \gamma) n$. Because $c^{\prime} \ll$ $\gamma \ll c \ll 1 / r$ and $\left|U \triangle U^{\prime}\right| \leqslant c^{\prime} n$, it is easy to see we can add vertices from $U$ to $W \cap U$ or delete vertices from $W \cap U$ to create either a $(1, c)$-independent or (2, $c$ )-independent set in $U$. This implies that $U$ is not $(s, c)$-tolerant.

A similar argument implies that when $s \in\{2,4\}$, if $G\left[U^{\prime}\right]$ is $(s, \gamma)$-splittable, then $U$ is not $(s, c)$-tolerant.

Claim 10.5 below is meant to capture all of the necessary facts about $(s, c)$ tolerant sets in a form that will be convenient. In some sense, Claim 10.5 is just a restatement of the main theorems from the previous sections.

With Claim 10.3, (i) and (ii) follow from Corollary 6.6, and (iii) and (iv) follow from Proposition 7.6 and Theorem 9.1, respectively. Note that, after the proof of this claim, we do not appeal to Corollary 6.6, Proposition 7.6 and Theorem 9.1 again.

Claim 10.5. Let $1 \leqslant s \leqslant r$ where $s \in \mathbb{N}$ and suppose $1 / n \ll \eta \ll \phi \ll \gamma \ll 1 / r$, $U \subseteq V$ such that $U$ is $(s, \gamma)$-tolerant and $U^{\prime} \subseteq V$ such that $\left|U \triangle U^{\prime}\right| \leqslant \eta n$.

(i) If $W \subseteq U^{\prime}$ such that $|W| \geqslant(s-1) n / r+\phi n$ then $G[W]$ contains a copy of $\overline{\mathcal{K}}_{s}$.

(ii) If $v \in V$ such that $d\left(v, U^{\prime}\right) \geqslant 2(s-1) n / r+\phi n$, then $G\left[U^{\prime}\right]$ contains a copy $T$ of $\bar{K}_{s}$ to which $v$ sends at least $2 s-1$ edges, so $G[T+v]$ contains a copy of $\hat{\mathcal{K}}_{s+1}$.

(iii) There exist at least $\phi n$ vertex-disjoint copies of $\mathcal{K}_{s+1}^{\prime}$ in $G\left[U^{\prime}\right]$.

(iv) If $\left|U^{\prime}\right|$ is divisible by $s$, then there exists a perfect $\mathcal{K}_{s}^{\prime}$-tiling in $G\left[U^{\prime}\right]$.

Proof. Note that, by Claim 10.3,

$$
\delta\left(G\left[U^{\prime}\right]\right) \geqslant 2(1-1 / s-r \eta / s)\left|U^{\prime}\right| .
$$


We first prove (i) and (ii), so let $W$ and $v$ be as in the statement of the claim. When $s \geqslant 2$, Corollary 6.6 and (15) imply that there exists a $\bar{K}_{s}$-tiling $\mathcal{T}$ of $G\left[U^{\prime}\right]$ on all but at most $4 s r \eta\left|U^{\prime}\right|$ vertices, and when $s=1$, this is trivially true. Let $Z:=V(\mathcal{T})$ be the vertices in $U^{\prime}$ that are covered by $\mathcal{T}$. Note that

$$
(s-1)|\mathcal{T}| \leqslant(s-1)\left|U^{\prime}\right| / s<(s-1) n / r+\phi n / 3 .
$$

Since

$|W \cap Z| \geqslant|W|-\left|U^{\prime} \backslash Z\right| \geqslant|W|-4 s r \eta\left|U^{\prime}\right|>(s-1) n / r+\phi n / 3>(s-1)|\mathcal{T}|$,

there exists $T \in \mathcal{T}$ such that $V(T) \subseteq W$, and this proves (i). Because

$$
d\left(v, U^{\prime}\right) \geqslant 2((s-1) n / r+\phi n / 3)+\phi n / 3>2(s-1)|\mathcal{T}|+2\left|U^{\prime} \backslash Z\right|
$$

there exists $T \in \mathcal{T}$ such that $d(v, V(T)) \geqslant 2 s-1$, so $v$ has at most one light neighbour in $T$. This proves (ii).

We now prove (iii) and (iv). To this end, let $U^{\prime \prime} \subseteq U^{\prime}$ such that

$$
\left|U^{\prime \prime}\right| \geqslant\left|U^{\prime}\right|-(s+1)\lceil\phi n\rceil .
$$

Note that

$$
\left|U^{\prime \prime} \triangle U\right| \leqslant\left|U^{\prime \prime} \triangle U^{\prime}\right|+\left|U^{\prime} \triangle U\right| \leqslant 4(s+1) \phi n / 3+\eta n \leqslant 3 s \phi n
$$

so, by Claim 10.3,

$$
\delta\left(G\left[U^{\prime \prime}\right]\right) \geqslant 2(1-1 / s-3 r \phi)\left|U^{\prime \prime}\right| .
$$

For $\gamma^{\prime}$ such that $1 / n \ll 3 r \phi \ll \gamma^{\prime} \ll \gamma \ll 1 / s$, by Claim 10.4 we have that $G\left[U^{\prime \prime}\right]$ is not $\left(1 / s, \gamma^{\prime}\right)$-extremal and when $s \in\{2,4\}$ is not $\left(s, \gamma^{\prime}\right)$-splittable. If $s=1$, (iv) is vacuously true. So suppose $s \geqslant 2$. Then Proposition 7.6 implies that $G\left[U^{\prime \prime}\right]$ has a perfect $\left(\mathcal{K}_{s}^{\prime} \cup \mathcal{K}_{s+1}^{\prime}\right)$-tiling and, when $s$ divides $\left|U^{\prime \prime}\right|$, Theorem 9.1 implies that $G\left[U^{\prime \prime}\right]$ has a perfect $\mathcal{K}_{s}^{\prime}$-tiling.

Taking $U^{\prime \prime}=U^{\prime}$ then gives (iv). Furthermore, we can greedily select $\lceil\phi n\rceil$ copies of elements from $\mathcal{K}_{s+1}^{\prime}$ from $G\left[U^{\prime}\right]$, since any subset of $U^{\prime}$ that has order greater than $\left|U^{\prime}\right|-(s+1)\lceil\phi n\rceil$ contains a copy of $\mathcal{K}_{s+1}^{\prime}$ : When $s \geqslant 2$, this is true because a perfect $\left(\mathcal{K}_{s}^{\prime} \cup \mathcal{K}_{s+1}^{\prime}\right)$-tiling in a multigraph of order not divisible by $s$ implies the existence of an element from $\mathcal{K}_{s+1}^{\prime}$, and, when $s=1$, this is true because $G\left[U^{\prime}\right]$ not being $\left(1, \gamma^{\prime}\right)$-extremal implies that $G\left[U^{\prime}\right]$ contains at least $\gamma^{\prime}\left|U^{\prime}\right|^{2}$ edges. This proves (iii). 
10.2. Initial partitioning and sorting. Suppose that

$$
0<1 / n \ll \gamma_{0} \ll \gamma_{1} \ll \cdots \ll \gamma_{r+2} \ll 1 / r
$$

and, in addition, for every $i \in[r+2]$, we have $\beta_{i}$ and $\psi_{i}$ such that

$$
\gamma_{i-1} \ll \beta_{i} \ll \psi_{i} \ll \gamma_{i} .
$$

We start by trying to find, for either $s_{1}=1$ or $s_{1}=2$, an $\left(s_{1}, \gamma_{1}\right)$-independent set which we call $A_{1}$. We then try to find, for $s_{2}=1$ or $s_{2}=2$, an $\left(s_{2}, \gamma_{2}\right)$-independent set $A_{2}$ disjoint from $A_{1}$. We continue in this manner for as long as possible, so in the end we have (a possibly empty, in which case $p=0$ ) collection of disjoint sets $A_{1}, \ldots, A_{p}$ and integers $s_{1}, \ldots, s_{p}$, such that $A_{i}$ is $\left(s_{i}, \gamma_{i}\right)$-independent for each $i \in[p]$. Let $U:=\overline{\bigcup_{i \in[p]} A_{i}}$ and set $s:=r-\left(s_{1}+\cdots+s_{p}\right)$. If $s=0$, then $U=\emptyset$ and we set $q:=0$ to indicate this case. If $U$ is $\left(s, \gamma_{p+1}\right)$-tolerant, then set $A_{p+1}:=U, s_{p+1}:=s$ and $q:=1$. Otherwise, $U$ is not $\left(s, \gamma_{p+1}\right)$-tolerant and because the initial process terminated, $U$ contains neither a $\left(1, \gamma_{p+1}\right)$-independent set nor a $\left(2, \gamma_{p+1}\right)$-independent set. By the definition of a tolerant set, it must therefore be that $s \in\{2,4\}$ and that $U$ has a partition $\left\{A_{p+1}, A_{p+2}\right\}$ that is $(s / 2$, $\left.\gamma_{p+1}\right)$-disconnected. We set $s_{p+1}:=s_{p+2}:=s / 2$ and $q:=2$, to indicate this case.

If $q=1$, set $\tau:=\gamma_{p}, \beta:=\beta_{p+1}, \psi:=\psi_{p+1}$ and $\gamma:=\gamma_{p+1}$. Otherwise, set $\tau:=\gamma_{p+1}, \beta:=\beta_{p+2}, \psi:=\psi_{p+2}$ and $\gamma:=\gamma_{p+2}$. Therefore,

$$
0<1 / n \ll \tau \ll \beta \ll \psi \ll \gamma \ll 1,
$$

and we have proved the following claim.

Claim 10.6. There exists a partition $\left\{A_{1}, \ldots, A_{p+q}\right\}$ of $V$ where $p+q \leqslant r$ and $q \in\{0,1,2\}$, and nonnegative integers $s, s_{1}, \ldots, s_{p+q}$ such that the following holds:

(i) $\left|A_{i}\right|=s_{i} n / r$ for $i \in[p+q]$;

(ii) for every $i \in[p], s_{i} \in\{1,2\}$ and $A_{i}$ is an $\left(s_{i}, \tau\right)$-independent set;

(iii) if $q=0$, then $s=0$;

(iv) if $q=1$, then $s=s_{p+1}$ and $A_{p+1}$ is $(s, \gamma)$-tolerant; and

(v) if $q=2$, then $s \in\{2,4\}$ and $\left\{A_{p+1}, A_{p+2}\right\}$ is an $(s / 2, \tau)$-disconnected pair.

Note the relationship between Claim 10.6 and the examples shown in Figure 2.

In order to discuss the case when $q=2$ and the case when $q \neq 2$ in a consistent way, we define a permutation $\sigma$ of $[p+q]$ in the following way. If $q \neq 2$ we let 
$\sigma$ be the identity permutation, and if $q=2$ we let $\sigma$ be the transposition of $p+1$ and $p+2$. Note that when $p+1 \leqslant i \leqslant p+q$, and $q \geqslant 1,\left|A_{i} \cup A_{\sigma(i)}\right|=s n / r$.

When $q \neq 1$, we let $\Lambda:=[p+q]$, and when $q=1$, we let $\Lambda:=[p]$. We say that a vertex $v$ is $(i, c)$-typical if $i \in \Lambda$ and

$$
s_{i}=1 \text { and } d\left(v, A_{\sigma(i)}\right) \leqslant c n \text {, or } s_{i}=2 \text { and } d^{2}\left(v, A_{\sigma(i)}\right) \leqslant c n ;
$$

or, if $q=1, i=p+1$ and $d^{2}\left(v, \overline{A_{p+1}}\right) \geqslant\left|\overline{A_{p+1}}\right|-c n$.

Claim 10.7. For any $i \in[p+q]$, if $v$ is $(i, c)$-typical, then $d^{2}\left(v, \overline{A_{\sigma(i)}}\right) \geqslant\left|\overline{A_{\sigma(i)}}\right|-$ $c n-1$, and, furthermore, if $i \in \Lambda$ and $s_{i}=2$, then $|N(v)| \geqslant(1-c) n-1$.

Proof. Consider any $i \in[p+q]$ and suppose $v$ is $(i, c)$-typical. If $q=1$ and $i=p+1$, then, recalling that $i=\sigma(i)=p+1$, we have $d^{2}\left(v, \overline{A_{\sigma(i)}}\right) \geqslant\left|\overline{A_{\sigma(i)}}\right|-c n$ by definition.

Otherwise, using (14), if $s_{i}=1$, we have that

$$
d^{2}\left(v, \overline{A_{\sigma(i)}}\right) \geqslant \delta(G)-\left|\overline{A_{\sigma(i)}}\right|-d\left(v, A_{\sigma(i)}\right) \geqslant\left|\overline{A_{\sigma(i)}}\right|-c n-1
$$

and if $s_{i}=2$, we have that

$$
d^{2}\left(v, \overline{A_{\sigma(i)}}\right) \geqslant \delta(G)-(n-1)-d^{2}\left(v, A_{\sigma(i)}\right) \geqslant\left|\overline{A_{\sigma(i)}}\right|-c n .
$$

When $s_{i}=2$, we also have that

$$
|N(v)| \geqslant \delta(G)-d^{2}(v) \geqslant \delta(G)-\left|\overline{A_{\sigma(i)}}\right|-c n \geqslant(1-c) n-1 .
$$

Claim 10.8. For any $0<c \leqslant 1$ and $i \in[p+q]$, there are at most $3 r \tau n / c$ vertices in $A_{i}$ that are not $(i, c)$-typical.

Proof. Let $i \in[p+q]$ and let $t$ be the number of vertices in $A_{i}$ that are not $(i, c)$-typical. If $i \in \Lambda$, then we have that

$$
\begin{array}{ll}
t c n \leqslant e\left(A_{i}, A_{\sigma(i)}\right) \leqslant 2 \tau n^{2} & \text { when } s_{i}=1, \quad \text { and }, \\
t c n \leqslant e_{2}\left(A_{i}, A_{\sigma(i)}\right) \leqslant 2 \tau n^{2} & \text { when } s_{i}=2,
\end{array}
$$

so $t \leqslant 2 \tau n / c$. Here we define $e\left(A_{i}, A_{i}\right):=2 e\left(A_{i}\right)$ and $e_{2}\left(A_{i}, A_{i}\right):=2 e_{2}\left(A_{i}\right)$.

If $q=1$ and $i=p+1$, then our assumption gives us

$$
\left|A_{p+1}\right|\left|\overline{A_{p+1}}\right|-t c n \geqslant e_{2}\left(A_{p+1}, \overline{A_{p+1}}\right),
$$


so if we can show that

$$
e_{2}\left(A_{p+1}, \overline{A_{p+1}}\right) \geqslant\left|A_{p+1}\right|\left|\overline{A_{p+1}}\right|-3 p \tau n^{2},
$$

this will imply $t \leqslant 3 p \tau n / c$, which will prove the claim. To show (17), let $j \in[p]$, and recall that $j=\sigma(j)$ and $\left|A_{j}\right| \in\{n / r, 2 n / r\}$. Clearly,

$$
e_{2}\left(A_{j}, \overline{A_{j}}\right) \geqslant\left|A_{j}\right| \delta(G)-e\left(A_{j}, A_{j}\right)-\left|A_{j}\right|\left|\overline{A_{j}}\right| \text {. }
$$

Therefore, when $\left|A_{j}\right|=n / r, \delta(G) \geqslant 2\left|\overline{A_{j}}\right|-1$, and $e\left(A_{j}, A_{j}\right) \leqslant 2 \tau n^{2}$, so

$$
e_{2}\left(A_{j}, \overline{A_{j}}\right) \geqslant\left|A_{j}\right|\left|\overline{A_{j}}\right|-\left|A_{j}\right|-2 \tau n^{2} \geqslant\left|A_{j}\right|\left|\overline{A_{j}}\right|-3 \tau n^{2}
$$

When $\left|A_{j}\right|=2 n / r, \delta(G) \geqslant 2\left|\overline{A_{j}}\right|+\left|A_{j}\right|-1$, and $e\left(A_{j}, A_{j}\right) \leqslant\left|A_{j}\right|^{2}+2 \tau n^{2}$, so

$$
e_{2}\left(A_{j}, \overline{A_{j}}\right) \geqslant\left|A_{j}\right|\left|\overline{A_{j}}\right|+\left|A_{j}\right|\left(\left|A_{j}\right|-1\right)-\left(\left|A_{j}\right|^{2}+2 \tau n^{2}\right) \geqslant\left|A_{j}\right|\left|\overline{A_{j}}\right|-3 \tau n^{2} .
$$

Therefore,

$$
\begin{aligned}
e_{2}\left(A_{p+1}, \overline{A_{p+1}}\right) & =\sum_{j=1}^{p} e_{2}\left(A_{p+1}, A_{j}\right) \geqslant \sum_{j=1}^{p}\left(\left|A_{p+1}\right|\left|A_{j}\right|-3 \tau n^{2}\right) \\
& =\left|A_{p+1}\right|\left|\overline{A_{p+1}}\right|-3 p \tau n^{2}
\end{aligned}
$$

Let $\mathcal{U}=\left(U_{1}, \ldots, U_{p+q}\right)$ be an ordered collection of $p+q$ pairwise disjoint subsets of $V$. We say that an $r$-set $T \subseteq \bigcup_{i=1}^{p+q} U_{i}$ is $\mathcal{U}$-balanced, if

- $G[T]$ contains a copy of $\mathcal{K}_{r}^{\prime}$;

- $\left|V(T) \cap U_{i}\right|=s_{i}$ for all $i \in[p]$; and

- $\left|V(T) \cap\left(U_{p+1} \cup U_{\sigma(p+1)}\right)\right|=s$ when $q>0$.

A set $T$ is called $\mathcal{U}$-well-balanced, if $T$ is $\mathcal{U}$-balanced, and when $q=2, T$ intersects exactly one of the two sets $U_{p+1}$ and $U_{p+2}$, that is for some $i \in\{p+1$, $p+2\},\left|V(T) \cap U_{i}\right|=s$ and $\left|V(T) \cap U_{\sigma(i)}\right|=0$. Note that when $q \neq 2$ every $\mathcal{U}$-balanced set is a $\mathcal{U}$-well-balanced set.

We say that a vertex is excellent for $i$ if it is $\left(i, \beta^{2}\right)$-typical and we say that a vertex is good for $i$ if it is $\left(i, \psi^{2}\right)$-typical. We make the following definitions:

$$
B_{i}:=\left\{v \in A_{i}: v \text { is excellent for } i\right\} \text { and } B:=\bigcup_{i \in[p+q]} B_{i}
$$


and we let

$$
C_{i}:=B_{i} \cup\{v \in \bar{B}: v \text { is good for } i\} \text { and } C:=\bigcup_{i \in[p+q]} C_{i} .
$$

We let $\mathcal{B}$ be the ordered collection $\left(B_{1}, \ldots, B_{p+q}\right)$ and $\mathcal{C}$ be the ordered collection $\left(C_{1}, \ldots, C_{p+q}\right)$. Note that, by Claim 10.7 , every vertex is good for at most one index $i \in[p+q]$, so the sets $C_{1}, \ldots, C_{p+q}$ are pairwise disjoint. Note that, for every $i \in[p+q]$, each vertex $v \in \bar{C}$ is not good for $i$, so it has a large number of edges into $B_{\sigma(i)}$. Since each vertex $v \in B_{i}$ is adjacent to almost everything in $\overline{B_{\sigma}(i)}$, we can argue below that there exists a set $T$ that is $\mathcal{B}$-well-balanced and such that $G[T+v]$ contains a copy of $\mathcal{K}_{r+1}^{\prime}$. As we see, this will allow us to easily distribute the vertices in $\bar{C}$ to sets in $\mathcal{C}$.

Claims 10.7 and 10.8 and the fact that $\tau \ll \beta \ll \psi \ll 1 / r$ imply the following claim.

CLAIM 10.9. The following holds:

(i) $|\bar{C}| \leqslant|\bar{B}| \leqslant \beta^{2} n$, and, in particular, for every $i \in[p+q]$,

$$
\left|A_{i} \triangle C_{i}\right|,\left|A_{i} \triangle B_{i}\right| \leqslant \beta^{2} n \quad \text { and } \quad\left|C_{i} \backslash B_{i}\right| \leqslant \beta^{2} n
$$

(ii) for every $i \in[p+q]$,

$v \in B_{i} \Longrightarrow d^{2}\left(v, \overline{B_{\sigma(i)}}\right) \geqslant\left|\overline{B_{\sigma(i)}}\right|-\beta n$, and

$v \in C_{i} \Longrightarrow d^{2}\left(v, \overline{B_{\sigma(i)}}\right) \geqslant\left|\overline{B_{\sigma(i)}}\right|-\psi n$;

(iii) for every $i \in \Lambda$, if $s_{i}=2$, then

$v \in B_{i} \Longrightarrow|N(v)| \geqslant(1-\beta) n \quad$ and $\quad v \in C_{i} \Longrightarrow|N(v)| \geqslant(1-\psi) n$.

Looking ahead, we construct an ordered collection $\mathcal{D}=\left(D_{1}, \ldots, D_{p+q}\right)$ such that $\left\{D_{1}, \ldots, D_{p+q}\right\}$ is a partition of $V$ and such that there exists a perfect $\mathcal{K}_{r}^{\prime}$ tiling in $G$ such that every element in the tiling is $\mathcal{D}$-well-balanced. This trivially implies that $\mathcal{D}$ must have the following properties:

(i) for every $i \in[p]$ and for $i=p+1$ when $q=1,\left|D_{i}\right|=s_{i} \cdot n / r$;

(ii) when $q=2$, both $\left|D_{p+1}\right|$ and $\left|D_{p+2}\right|$ are divisible by $s$ and $\left|D_{p+1} \cup D_{p+2}\right|=$ $s n / r$.

For any multigraph $G^{\prime} \subseteq G$, call $\mathcal{D}=\left(D_{1}^{\prime}, \ldots, D_{p+q}^{\prime}\right)$ a proper ordered collection of $G^{\prime}$ if the sets in $\mathcal{D}$ form a partition of $V\left(G^{\prime}\right)$ and it meets conditions (i) and (ii) (with $n$ replaced by $\left|G^{\prime}\right|$ ). Let $c_{i}:=\left|C_{i}\right|-s_{i} n / r$ for every $i \in[p+q]$, so $|\bar{C}|+c_{1}+\cdots+c_{p+q}=0$. 
In order to make the rest of the section easier to understand, we now give a brief, informal description of the remainder proof for the case when $q \neq 2$. It is very similar to the approach taken by Komlós, Sárközy and Szemerédi in their proof of the Alon-Yuster conjecture [17]. We ignore the case when $q=2$ in this description to avoid technical details.

Our main goal is to balance the sizes of the sets $C_{1}, \ldots, C_{p+q}$. We begin by considering the sets that are too large, that is for every $i \in[p+q]$ such that $c_{i}>0$, we move exactly $c_{i}$ vertices out of $C_{i}$ to form the set $D_{i}$. We want to ensure that the vertices which are moved out of $C_{i}$ can eventually be covered by a copy of $\mathcal{K}_{r}^{\prime}$ that has $s_{i}$ other vertices in $C_{i}$. Therefore, we argue that we can find a $\mathcal{K}_{s_{i}+1}^{\prime}$-tiling in $G\left[C_{i}\right]$ of size $c_{i}$. From each element of this tiling we arbitrarily select a vertex $v$ to remove from $C_{i}$ when forming $D_{i}$, and, temporarily, place $v$ into a set we call $F^{\prime}$. Then we extend each element of this tiling to form a copy of $\mathcal{K}_{r+1}^{\prime}$ that has exactly $s_{j}$ vertices in $C_{j}$ for each $j \in[p+q] \backslash i$, and use the label $T_{v}$ for this copy of $\mathcal{K}_{r+1}^{\prime}$. So $T_{v}-v$ is $\mathcal{C}$-well-balanced . We let $\mathcal{T}=\left\{T_{v}: v \in F^{\prime}\right\}$. Note that, after this process has completed, we have that, for every $c_{i} \geqslant 0, D_{i}=C_{i} \backslash F^{\prime}$ and $\left|D_{i}\right|=s_{i} n / r$.

Next, we prepare to distribute the vertices that were not assigned to some set $C_{i}$. To do this, for every such $v \in \bar{C}$, we find a $\mathcal{B}$-well-balanced $r$-set $T^{\prime}$ such that $G\left[T^{\prime}+v\right]$ contains an element of $\mathcal{K}_{r+1}^{\prime}$. We then label $T^{\prime}+v$ as $T_{v}$, and add $T_{v}$ to $\mathcal{T}$. Throughout, we ensure that the elements in $\mathcal{T}$ are vertex-disjoint. We let $F=F^{\prime} \cup \bar{C}$.

Note that by Claim 10.9(i),

$$
|F|=\sum_{i \in[p+q] ; c_{i}<0}-c_{i} \leqslant|\bar{B}| \leqslant \beta^{2} n .
$$

So we can distribute the small number of vertices in $F$ arbitrarily to every $D_{i}$ such that $\left|D_{i}\right|<s_{i} n / r$ until $\left|D_{i}\right|=s_{i} n / r$ for every $i \in[p+q]$. Suppose $v \in F$ has been assigned to $D_{i}$; so $T_{v}$ has exactly $s_{i}$ vertices in $C_{i}$ and, with $v$, has $s_{i}+1$ vertices in $D_{i}$. We can then arbitrarily remove one element from $T_{v} \cap C_{i}$ to create a $\mathcal{D}$-well-balanced set. After this has been done for every $v \in F$, we have that $\mathcal{T}$ is a $\mathcal{K}_{r}^{\prime}$-tiling in which every element corresponds to a $\mathcal{D}$-well-balanced $r$-set. We let $G^{\prime}=G-V(\mathcal{T})$ and $D_{i}^{\prime}=D_{i} \backslash V(\mathcal{T})$ for every $i \in[p+q]$. So $\left(D_{1}^{\prime}, \ldots, D_{p+q}^{\prime}\right)$ is a proper ordered collection of $G^{\prime}$ and $D_{i}^{\prime} \subseteq C_{i}$ for every $i \in[p+q]$. Claim 10.10 below will then complete the proof. When $q=2$, our approach is similar. The main difference is that we have to be somewhat careful to ensure that $\left|D_{p+1}\right|$ and $\left|D_{p+2}\right|$ are both divisible by $s$. The details for all cases are in Section 10.3.

We now continue the proof of Theorem 10.1 by proving Claim 10.10 which will be used at the very end of the proof to construct the vast majority of elements of our tiling of $G$. 
ClaIM 10.10. Let $G^{\prime}$ be an induced subgraph of $G$ such that $\left|G^{\prime}\right| \geqslant|G|-\beta$, and let $\mathcal{D}^{\prime}=\left(D_{1}^{\prime}, \ldots, D_{p+q}^{\prime}\right)$ be a proper ordered collection of $V\left(G^{\prime}\right)$. If $D_{i}^{\prime} \subseteq C_{i}$ for every $i \in[p+q]$, then there exists a perfect $\mathcal{K}_{r}^{\prime}$-tiling in $G^{\prime}$.

Proof. For $i \in[p]$, we let $\mathcal{T}_{i}$ be a perfect $\overline{\mathcal{K}}_{s_{i}}$-tiling of $G\left[D_{i}^{\prime}\right]$. When $s_{i}=1$ such a tiling trivially exists, and, when $s_{i}=2$, it exists by Claim 10.9(iii). When $q \geqslant 1$, we let $\mathcal{T}_{p+1}$ be a perfect $\mathcal{K}_{s}^{\prime}$-tiling of $G\left[D_{p+1}^{\prime} \cup D_{\sigma(p+1)}^{\prime}\right]$. By Claim 10.9(ii), this is easy to find when $q=2$ by, say, applying the Hajnal-Szemerédi theorem to the graph induced by the heavy edges of $D_{p+1}^{\prime}$ and then to the graph induced by the heavy edges of $D_{p+2}^{\prime}$. (Actually the use of the Hajnal-Szemerédi theorem here is overkill; it is very easy to directly argue the desired perfect tiling in $G\left[D_{p+1}^{\prime} \cup\right.$ $\left.D_{\sigma(p+1)}^{\prime}\right]$ exists.) When $q=1$, it is implied by Claim 10.5(iv), because $\beta \ll \gamma$ and

$$
\left|A_{p+1} \triangle D_{p+1}^{\prime}\right| \leqslant\left|A_{p+1} \triangle C_{p+1}\right|+\left(|G|-\left|G^{\prime}\right|\right) \leqslant\left(\beta^{2}+\beta\right) n .
$$

Let $t=p$ when $q=0$, or let $t=p+1$, when $q \geqslant 1$, and let $H$ be a $t$-partite graph with vertex classes $\mathcal{T}_{1}, \ldots, \mathcal{T}_{t}$ such that, for every distinct $i, i^{\prime} \in[t], T \in \mathcal{T}_{i}$ and $T^{\prime} \in \mathcal{T}_{i^{\prime}}, T$ is adjacent to $T^{\prime}$ when every vertex in $T$ is heavily adjacent to every vertex in $T^{\prime}$. Note that $H$ is balanced with each vertex class of size $m=\left|G^{\prime}\right| / r$. So we are done if there is a perfect $K_{t}$-tiling in $H$. By Claim 10.9(ii), when $i, i^{\prime}$ are distinct and $T \in \mathcal{T}_{i}$, the number of neighbours of $T$ in $\mathcal{T}_{i^{\prime}}$ is at least

$$
m-\left|\bigcup_{u \in T}\left(D_{i^{\prime}}^{\prime} \backslash N^{2}(u)\right)\right| \geqslant m-r \psi n \geqslant\left(1-2 r^{2} \psi\right) m .
$$

For some $1 \leqslant t^{\prime}<t$, assume we have a perfect $K_{t^{\prime}}$-tiling $\mathcal{K}$ of $H\left[\mathcal{T}_{1} \cup \cdots \cup \mathcal{T}_{t^{\prime}}\right]$. We extend $\mathcal{K}$ to a perfect $K_{t^{\prime}+1}$-tiling of $H\left[\mathcal{T}_{1} \cup \cdots \cup \mathcal{T}_{t^{\prime}+1}\right]$ by finding a perfect matching in the bipartite graph with vertex classes $\mathcal{K}$ and $\mathcal{T}_{t^{\prime}+1}$ in which $K \in \mathcal{K}$ is adjacent to $T \in \mathcal{T}_{t^{\prime}+1}$ when $K+T$ is a copy of $K_{t^{\prime}+1}$ in $H$. By (18), this bipartite graph has minimum degree at least $\left(1-2 r^{2} t^{\prime} \psi\right) m \geqslant m / 2$, so Hall's theorem implies that it has a perfect matching. Since this holds for every $t^{\prime}<t$, there exists a perfect $K_{t}$-tiling in $H$.

In Claim 10.11(i) below we establish that, for some $k \in[p+q]$, we can extend a copy of $\mathcal{K}_{s_{k}+1}^{\prime}$ (respectively $\mathcal{K}_{s_{k}}^{\prime}$ ) that is contained in some $C_{k}$ where $k \leqslant p$ or extend a copy of $\mathcal{K}_{s+1}^{\prime}$ (respectively $\mathcal{K}_{s}^{\prime}$ ) contained in $C_{p+1} \cup C_{\sigma(p+1)}$ to a copy of $\mathcal{K}_{r+1}^{\prime}$ (respectively $\mathcal{K}_{r}^{\prime}$ ) with the correct number of vertices in every $C_{j}$ for $j \in[p+q]-i$. This is used when we remove elements from the sets $C_{k}$ that are too large as described in the overview above. When $q=2$, we use Claim 10.11(i) with $t=0$ when $\left|C_{p+1} \cup C_{p+2}\right| \geqslant s n / r$ to move vertices between $C_{p+1}$ to $C_{p+2}$ to make the order of both sets divisible by $s$. The second part of the lemma, (ii), 
which is only used when $q=2$, is similar but we start with a copy of $\mathcal{K}_{s_{k}+1}^{\prime}$ in some $C_{k}$ with $k \leqslant p$ and extend it only into a copy of $\mathcal{K}_{r}^{\prime}$. Furthermore, for any $0 \leqslant \ell \leqslant s-1$, this $\mathcal{K}_{r}^{\prime}$ will have exactly $\ell$ vertices in $C_{p+1}$ and $s-\ell-1$ vertices in $C_{p+2}$. This is used because sometimes when we must move a vertex from some $C_{k}$ with order greater than $s_{k} n / r$, to $C_{p+1}$ because the order of $C_{p+1} \cup C_{p+2}$ is slightly less than $s n / r$, we may also have to move some vertices in $C_{p+2}$ to $C_{p+1}$ to ensure that both sets are divisible by $s$.

Claim 10.11. Let $W \subseteq V$ such that $|W| \leqslant \beta n$, let $k \in[p+q]$ and $t \in\{0,1\}$. When $k \leqslant p$, let $S \subseteq C_{k}$ such that $|S|=s_{k}+t$ and when $k \geqslant p+1$, let $S \subseteq$ $C_{k} \cup C_{\sigma(k)}$ such that $|S|=s+t$. Suppose that $G[S]$ contains an element of $\mathcal{K}_{|S|}^{\prime}$.

(i) There exists a set $T$ that avoids $W$ such that $T \cup S$ is $\mathcal{C}$-balanced when $t=0$, or, when $t=1, T \cup(S-v)$ is $\mathcal{C}$-balanced for any $v \in S$. Furthermore, when $q=2$, the set $T$ does not intersect $C_{p+2}$.

(ii) When $q=2, t=1$, and $k \notin\{p+1, p+2\}$, for any $j \in\{p+1, p+2\}$ and $1 \leqslant \ell \leqslant s-1$, there exists a set $T$ that avoids $W$ such that $G[T \cup S]$ contains an element of $\mathcal{K}_{r}^{\prime},\left|T \cap C_{j}\right|=\ell,\left|T \cap C_{\sigma(j)}\right|=s-1-\ell$ and $\left|T \cap C_{i}\right|=s_{i}$ for each $i \in[p]-k$.

Proof. We construct $T$ iteratively, and throughout, we let

$$
C_{i}^{\prime}:=\left(C_{i} \backslash W\right) \cap\left(\bigcap_{u \in T \cup S} N^{2}(u)\right) .
$$

Assuming $T \subseteq C$, Claim 10.9(ii) implies that for all $i$ such that $C_{\sigma(i)} \cap(S \cup T)$ $=\emptyset$,

$$
\left|C_{i}^{\prime}\right| \geqslant\left|C_{i}\right|-|W|-|T \cup S| \psi n \geqslant\left|C_{i}\right|-2 r \psi n .
$$

We start the construction by adding vertices from $C_{p+1}^{\prime} \cup C_{\sigma(p+1)}^{\prime}$ to $T$. If $q=0$, or $q \geqslant 1$ and $k \geqslant p+1$, we do not add any vertices from $C_{p+1}^{\prime} \cup C_{\sigma(p+1)}^{\prime}$ to $T$. Otherwise, we know $\left|C_{p+1}^{\prime} \cup C_{\sigma(p+1)}^{\prime}\right|$ is large by (19) since $\left(C_{p+1} \cup C_{\sigma(p+1)}\right) \cap S=$ $\emptyset$. If $q=1$, then, since $\beta \ll \psi \ll \gamma$, Claim 10.5(i) and Claim 10.9(i) imply that we can let $T \subseteq C_{p+1}^{\prime}$ such that $|T|=s$ and $G[T]$ contains an element of $\overline{\mathcal{K}}_{s}$. To prove (i) when $q=2$, note that (19) and Claim 10.9(ii), imply that we can let $T$ induce a clique of size $s$ on heavy edges in $C_{p+1}^{\prime}$. To prove (ii), we start by using (19) and Claim 10.9(ii) to find vertices $u_{1}, \ldots, u_{\ell} \in C_{j}^{\prime}$ that form a clique on heavy edges and add these vertices to $T$. If $s-1-\ell=0$, we are done, so assume that this is not the case, which implies that $s=4$. Therefore, Claim 10.9(ii) and Claim 10.9(iii), imply that we can find $s-1-\ell$ vertices in $N\left(u_{1}\right) \cap \cdots \cap N\left(u_{\ell}\right) \cap C_{\sigma(j)}^{\prime}$ that form a clique on heavy edges. We then add these 
vertices to $T$. Note that in this case when $s=4$ either $\ell=1$ and $s-1-\ell=2$ or $\ell=2$ and $s-1-\ell=1$. So in this case currently $G[T]$ contains all possible edges except that it may have a light path on 3 vertices.

Now, for every $i \in\{1, \ldots, p\}-k$, in turn we use (19), to add either one vertex, when $s_{i}=1$, or two adjacent vertices, when $s_{i}=2$, from $C_{i}^{\prime}$ to $T$. When $s_{i}=2$ we can easily find two adjacent vertices in $C_{i}^{\prime}$ using Claim 10.9(iii). Further, note that by definition of $C_{i}^{\prime}$, in this step any selected vertices in $C_{i}^{\prime}$ send heavy edges to all previously selected elements of $S \cup T$. This ensures that there are all possible edges in $G[S \cup T]$ except for perhaps a collection of vertex-disjoint light edges, and at most one path on 3 vertices (in the case when $s=4$ ). That is, $G[S \cup T]$ is a copy of $\mathcal{K}_{|S \cup T|}^{\prime}$. It is now easy to see that the claim holds.

The main purpose of Claim 10.12(i) is to help distribute the vertices $v \in \bar{C}$. We construct a $\mathcal{B}$-well-balanced set $T$ such that $G[T+v]$ contains an element of $\mathcal{K}_{r+1}^{\prime}$; thus we can then move $v$ to any set $D_{i}$ and remove any element in $B_{i} \cap T$ from $T$ leaving a $\mathcal{D}$-well-balanced set. When $q=2$, we may need to move some $v \in \bar{C}$ to one of $D_{p+j}$ for $j \in\{1,2\}$ and, at the same time, move some vertices from $D_{p+3-j}$ to $D_{p+j}$ to ensure that $D_{p+1}$ and $D_{p+2}$ are both divisible by $s$. This is the reason for Claim 10.12(ii). Note that the following claim is essentially the reason we define both the sets $B_{1}, \ldots, B_{p+q}$ and the sets $C_{1}, \ldots, C_{p+q}$.

Claim 10.12. Let $W \subseteq V$ such that $|W| \leqslant \beta n$ and $v \in \bar{C}$.

(i) There exists a $\mathcal{B}$-well-balanced set $T$ that avoids $W$ such that $G[T+v] \in$ $\mathcal{K}_{r+1}^{\prime}$.

(ii) When $q=2$, for $j \in\{p+1, p+2\}$ and $1 \leqslant \ell \leqslant s-1$, there exists a set $T$ that avoids $W$ such that $G[T+v]$ contains an element of $\mathcal{K}_{r}^{\prime},\left|T \cap B_{j}\right|=\ell$, $\left|T \cap B_{\sigma(j)}\right|=s-1-\ell$ and $\left|T \cap B_{i}\right|=s_{i}$ for each $i \in[p]$.

Proof. For any $i \in[p+q]$, if $i \in \Lambda$, since $v$ it not (i, $\left.\psi^{2}\right)$-typical, Claim 10.9(i) implies that

when $s_{i}=1, d\left(v, B_{i}\right) \geqslant \psi^{2} n / 2, \quad$ and, when $s_{i}=2, d^{2}\left(v, B_{i}\right) \geqslant \psi^{2} n / 2$.

Similarly, when $q=1$, since $v$ is not $\left(p+1, \psi^{2}\right)$-typical, $d^{2}\left(v, \overline{A_{p+1}}\right) \leqslant\left|\overline{A_{p+1}}\right|-$ $\psi^{2} n$ and

$$
\begin{aligned}
d\left(v, B_{p+1}\right) & \geqslant \delta(G)-d\left(v, \overline{B_{p+1}}\right) \\
& \geqslant \delta(G)-\left(\left|\overline{A_{p+1}}\right|+d^{2}\left(v, \overline{A_{p+1}}\right)+2\left|\overline{B_{p+1}} \backslash \overline{A_{p+1}}\right|\right) \\
& \geqslant 2(1-1 / s) s n / r+\psi^{2} n / 2 .
\end{aligned}
$$


For $i \in[p+q]$, let $B_{i}^{\prime}:=B_{i} \backslash W$. We have that, by Claim 10.9(i),

$$
\left|A_{i} \triangle B_{i}^{\prime}\right| \leqslant\left|A_{i} \triangle B_{i}\right|+|W| \leqslant 2 \beta n .
$$

Let

$$
I:=\left\{i \in \Lambda: s_{i}=1 \text { and } d^{2}\left(v, B_{i}\right) \leqslant \psi^{2} n / 2\right\}
$$

and note that there are at least $\sum_{i \in I}\left(\left|B_{i}\right|-\psi^{2} n / 2\right) \geqslant|I|\left(1 / r-\psi^{2}\right) n$ vertices in $\bigcup_{i \in I} B_{i}$ that are not heavy neighbours of $v$. By (14), when $r>2, d^{2}(v) \geqslant$ $\delta(G)-(n-1) \geqslant r \psi n / 2$, so there exists $j \in[p+q] \backslash I$, and

$$
\begin{aligned}
d^{2}\left(v, B_{j}\right) & \geqslant \delta(G)-(n-1)-\left(\left|\overline{B_{j}}\right|-|I|\left(1 / r-\psi^{2}\right) n\right) \\
& \geqslant\left|B_{j}\right|-\left(2 / r-|I| / r+|I| \psi^{2}\right) n .
\end{aligned}
$$

Hence, in all cases, $|I| \leqslant 2$, and, for any $j \in[p+q] \backslash I$,

$$
d^{2}\left(v, B_{j}\right) \geqslant\left|B_{j}\right|-2 \psi^{2} n \quad \text { if }|I|=2 .
$$

We prove (i) and (ii) simultaneously. Let $t \in\{0,1\}$, so that $s-1+t$ is the number of vertices of $T$ that will intersect $B_{p+1} \cup B_{\sigma(p+1)}$, that is when we are proving (i) we have $t=1$ and when we are proving (ii) we have $t=0$.

We now give a brief overview of our plan for constructing the set $T$. Clearly we must construct $T$ so that every pair of vertices in $T+v$ are adjacent. We also have that

$\left(\alpha_{1}\right)$ the only light edges in $G[T+v]$ that are not incident to $v$, are in the subgraphs $G\left[T \cap B_{i}^{\prime}\right]$ where $s_{i}=2$, or are in $G\left[T \cap\left(B_{p+1}^{\prime} \cup B_{\sigma(p+1)}^{\prime}\right)\right]$ when $q \geqslant 1$;

$\left(\alpha_{2}\right) v$ is heavily adjacent to every vertex $u$ in $T \cap B_{i}^{\prime}$ when $i \in \Lambda \backslash I$;

$\left(\alpha_{3}\right)$ if $q \geqslant 1, G\left[T \cap\left(B_{p+1}^{\prime} \cup B_{\sigma(p+1)}^{\prime}\right)+v\right]$ will contain an element of $\mathcal{K}_{s-1+t}^{\prime}$.

If $v$ has at most one light neighbour in $T$, this is enough to give us that $G[T+v]$ contains an element of $\mathcal{K}_{r+t}^{\prime}$, which would prove the claim. However, we can only ensure that $v$ has at most two light neighbours in $T$. To prove the claim, we then also meet one of the following conditions when $v$ has two light neighbours in $T$ :

$\left(\beta_{1}\right)$ the two light neighbours of $v$ are in different sets $B_{i}^{\prime}$ such that $i \in I$ and $i \leqslant p$ and if $q \geqslant 1, G\left[T \cap\left(B_{p+1}^{\prime} \cup B_{\sigma(p+1)}^{\prime}\right)\right]$ contains an element of $\overline{\mathcal{K}}_{s-1+t}$ except possibly when $q=2, s=4, t=0$ and $\ell \in\{1,2\}$, and in this case it contains an element of $\hat{\mathcal{K}}_{s-1+t}$; or

$\left(\beta_{2}\right) q=2, s=2, t=1, I=\{p+1, p+2\}$ and the two light neighbours of $v$ are in some $B_{i}^{\prime}$ where $i \in I$ and these two light neighbours are heavily adjacent; or 
$\left(\beta_{3}\right) q=2, s=2, t=0,|I|=2, j \in I \cap\{p+1, p+2\}, v$ has one light neighbour in $B_{j}^{\prime}$ and the other light neighbour is in some $B_{i}^{\prime}$ where $i \in I$ and $i \leqslant p$; or

$\left(\beta_{4}\right) q=1,|I|=1$, one light neighbour of $v$ is in the set $B_{i}^{\prime}$ such that $i \in I$ and the other light neighbour of $v$ is in $B_{p+1}^{\prime}$ and $G\left[T \cap B_{p+1}^{\prime}+v\right]$ contains an element of $\hat{\mathcal{K}}_{s+t}$.

We build the set $T$ iteratively. We start by adding vertices from $B_{p+1}^{\prime} \cup B_{\sigma(p+1)}^{\prime}$ to $T$, so when $q=0$ we do not add anything to $T$. Recall that $\beta \ll \psi \ll \gamma$. If $q=1$ and $|I| \leqslant 1$, then (20), (21) and Claim 10.5(ii), imply that we can choose $T \subseteq B_{p+1}^{\prime}$, such that $|T|=s, G[T+v]$ contains an element of $\hat{\mathcal{K}}_{s+1}$ and in which $v$ has one light neighbour. If $q=1$ and $|I|=2$, then by (21), (22), and Claim 10.5(i) we can let $T \subseteq B_{p+1}^{\prime}$ be such that $T \subseteq N^{2}(v)$ and $G[T]$ contains an element of $\overline{\mathcal{K}}_{s}$.

Now assume $q=2$. To prove (i), when $I=\{p+1, p+2\}$, we pick $j \in\{p+1$, $p+2\}$ arbitrarily, otherwise we let $j \in\{p+1, p+2\} \backslash I$. Recall that $j \in I$ implies that $s=2$. Let $Z:=N(v) \cap B_{j}^{\prime}$ when $j \in I$ and let $Z:=N^{2}(v) \cap B_{j}^{\prime}$ when $j \notin I$. Note that, $|Z| \geqslant \psi^{2} n / 2-|W| \geqslant \psi^{2} n / 3$, in either case. We now use Claim 10.9(ii) to find an $s$-set $T \subseteq Z$ such that $T$ induces a clique on heavy edges in $G$.

To prove (ii), we assume $j \in\{p+1, p+2\}$ is given. Note that if $j \in I$, then we must have that $s=2$ and $\ell=1$. Again, we let $Z:=N(v) \cap B_{j}^{\prime}$ when $j \in I$ and let $Z:=N^{2}(v) \cap B_{j}^{\prime}$ when $j \notin I$, so $|Z| \geqslant \psi^{2} n / 3$. Now we use Claim 10.9(ii) to find vertices $u_{1}, \ldots, u_{\ell} \in Z$ such that they induce a clique on heavy edges in $G$, and we add these vertices to $T$. If $s-1-\ell=0$, we are done, so assume that this is not the case, which implies that $s=4$. Therefore, Claim 10.9(iii) and Claim 10.9(ii), imply that we can find $s-1-\ell$ vertices in $N\left(u_{1}\right) \cap \cdots \cap N\left(u_{\ell}\right) \cap N^{2}(v) \cap B_{\sigma(j)}^{\prime}$ that induce a clique on heavy edges in $G$. We then add these vertices to $T$. If $\ell=\{1,2\}$, then $G[T]$ contains an element of $\hat{K}_{3}$ and when $\ell=3$ it is a clique on heavy edges.

Note that in all cases the way we have constructed $T$ so far ensures that $\left(\alpha_{3}\right)$ holds.

Now, in turn for each $i$ with $1 \leqslant i \leqslant p$, we add $s_{i}$ vertices from $B_{i}^{\prime}$ to $T$. At each step, when $i \in I$, we let

$$
\begin{aligned}
& Z:=B_{i}^{\prime} \cap N(v) \cap\left(\bigcap_{u \in T} N^{2}(u)\right), \quad \text { and otherwise let } \\
& Z:=B_{i}^{\prime} \cap N^{2}(v) \cap\left(\bigcap_{u \in T} N^{2}(u)\right) .
\end{aligned}
$$


(So the definition of $Z$ gets updated at each step, as we add more elements to $T$.) Note that, with Claim 10.9(ii), $|Z| \geqslant \psi^{2} n / 2-|W|-r \beta n \geqslant \psi^{2} n / 3$ in both cases. When $s_{i}=1$, we add one vertex from $Z$ to $T$, and when $s_{i}=2$, we can add two adjacent vertices in $Z$ to $T$, since Claim 10.9(iii) implies that there exists an edge in $G[Z]$.

This completes the construction of $T$. Note that $\left(\alpha_{1}\right)$ and $\left(\alpha_{2}\right)$ immediately hold. Further, one of $\left(\beta_{1}\right)-\left(\beta_{4}\right)$ holds in each case. It is easy to see that in any case we obtain a set $T$ as desired.

10.3. Finishing the proof. We now finish the proof by constructing $\mathcal{D}=$ $\left(D_{1}, \ldots, D_{p+q}\right)$ a proper ordered collection of $G$ and a collection $\mathcal{T}$ of vertexdisjoint $\mathcal{D}$-well-balanced sets as described above. We build the collection $\mathcal{T}$ iteratively, and, at times, it may include $(r+1)$-sets, as well as $r$-sets.

Let $c_{i}:=\left|C_{i}\right|-s_{i} n / r$ for every $i \in[p+q]$. For each $i \in[p]$ such that $c_{i} \geqslant 0$, we find a $\hat{K}_{s_{i}+1}$-tiling $\mathcal{S}_{i}$ of $G\left[C_{i}\right]$ containing exactly $c_{i}$ elements. When $s_{i}=1$, each vertex in $C_{i}$ has at least

$$
\left\lceil\left(\delta(G)-2\left|\overline{C_{i}}\right|\right) / 2\right\rceil \geqslant\left|C_{i}\right|-n / r=c_{i}
$$

neighbours in $C_{i}$. Therefore, by Lemma 10.2, we can let $\mathcal{S}_{i}$ be a matching of size $c_{i}$ in $G\left[C_{i}\right]$. Similarly, for $i \in[p]$ such that $s_{i}=2$ and $\left|C_{i}\right| \geqslant 2 n / r$, there exists a matching $M_{i}$ containing at least

$$
\delta(G)-(n-1)-\left|\overline{C_{i}}\right| \geqslant\left|C_{i}\right|-2 n / r=c_{i}
$$

heavy edges in $G\left[C_{i}\right]$, and, by Claim 10.9(iii), we can pair each edge in $e \in M_{i}$ to a distinct vertex $v_{e}$ such that $v_{e}$ is a neighbour of both endpoints of $e$. Therefore, we have a collection $\mathcal{S}_{i}$ of $c_{i}$ vertex-disjoint elements of $\hat{\mathcal{K}}_{3}$ in $G\left[C_{i}\right]$. We let $\mathcal{S}$ be the union of the sets $\mathcal{S}_{i}$ constructed so far.

If $q=1$ and $\left|C_{p+1}\right|>s n / r$, we can use Claim 10.5(iii) to find a $\mathcal{K}_{s+1}^{\prime}$-tiling of size $c_{p+1}=\left|C_{p+1}\right|-s n / r$ in $G\left[C_{p+1}\right]$, we call this tiling $\mathcal{S}_{p+1}$ and we add it to $\mathcal{S}$.

Now suppose $q=2$ and $\left|C_{p+1} \cup C_{p+2}\right| \geqslant s n / r$; we find a tiling consisting of exactly $\left|C_{p+1} \cup C_{p+2}\right|-s n / r$ copies of $\mathcal{K}_{s+1}^{\prime}$ in $C_{p+1}$. Note that this is trivial to do, by Claim 10.9(ii). Indeed, we can easily find $\left|C_{p+1} \cup C_{p+2}\right|-s n / r$ vertexdisjoint $(s+1)$-sets in $C_{p+1}$, each forming a clique in the heavy edges of $G\left[C_{p+1}\right]$. We call this set $\mathcal{S}_{p+1}$ and we add the sets in $\mathcal{S}_{p+1}$, to $\mathcal{S}$. Note that there is slack in the argument here: given a single fixed set $X \subseteq V$ where $|X| \leqslant \gamma n$ we can additionally ensure no tile in $\mathcal{S}_{p+1}$ intersects $X$. We use this property shortly.

We have now completely defined $\mathcal{S}$. Our next goal is to construct the sets $D_{1}$, $\ldots, D_{p+q}$ such that $\mathcal{D}=\left(D_{1}, \ldots, D_{p+q}\right)$ is a proper ordered collection of $G$. We first define the $D_{i}$ for $i$ such that $c_{i} \geqslant 0$. Once we have done this we then define the remaining $D_{i}$. 
Suppose that $q=2$ and $\left|C_{p+1} \cup C_{p+2}\right| \geqslant s n / r$. Remove $c_{p+1}$ vertices from $C_{p+1}$ and call the resulting set $D_{p+1}$; we do this in such a way that these removed vertices consist of precisely one vertex from each tile in $\mathcal{S}_{p+1}$. Place these $c_{p+1}$ vertices into a set $F^{\prime}$. Let $D_{p+2}:=C_{p+2}$. This ensures that $\left|D_{p+1} \cup D_{p+2}\right|=s n / r$. However, we also need to ensure that both $\left|D_{p+1}\right|$ and $\left|D_{p+2}\right|$ are divisible by $s$. Therefore, we find a set $X \subseteq C_{p+1}$ and a set $Y \subseteq C_{p+2}$ such that, $|X \cup Y|=s$, $G[X \cup Y]$ contains an element of $\mathcal{K}_{s}^{\prime}$, and

$$
|Y| \equiv\left|C_{p+2}\right| \quad(\bmod s) .
$$

We then move the vertices in $Y$ from $D_{p+2}$ to $D_{p+1}$. We need the exact minimum degree condition to construct these sets $X, Y$. So actually formally what we do is first construct $X$ and $Y$ then the collection $\mathcal{S}_{p+1}$ as before such that $\mathcal{S}_{p+1}$ is disjoint from the set $X$. Also, for consistency, we construct the sets $X$ and $Y$ even when there is no divisibility issue, that is when $\left|C_{p+2}\right|$ is divisible by $s$. In this case, we let $Y=\emptyset$ and $X$ be a clique on $s$ vertices in the heavy edges of $G\left[C_{p+1}\right]$, which can be found easily using Claim 10.9(ii). Therefore, it only remains to show how we construct $X$ and $Y$ when $\left|C_{p+2}\right|$ is not divisible by $s$. If $s=2$, then, for some $i \in\{p+1, p+2\},\left|C_{i}\right| \geqslant n / r$, so $\delta(G)-2\left(\left|\overline{C_{i}}\right|-1\right) \geqslant 1$, which implies that every vertex in $\overline{C_{i}}$ has a neighbour in $C_{i}$, and, in particular, there exists an edge $x y$ such that $x \in C_{p+1}$ and $y \in C_{p+2}$, and we let $X:=\{x\}$ and $Y:=\{y\}$. If $s=4$, then for some $i \in\{p+1, p+2\},\left|C_{i}\right| \geqslant 2 n / r$, and $\delta(G)-(n-1)-\left(\left|\overline{C_{i}}\right|-1\right) \geqslant 1$, which implies that every vertex in $\overline{C_{i}}$ has a heavy neighbour in $C_{i}$ and we can let $x_{1} y_{1}$ be a heavy edge such that $x_{1} \in C_{p+1}$ and $y_{1} \in C_{p+2}$. Let $j$ be such that $1 \leqslant j \leqslant 3$ and $j \equiv\left|C_{p+2}\right|(\bmod 4)$. By Claim 10.9(ii)-(iii), we can find vertices $y_{2}, \ldots, y_{j}$ in $N\left(x_{1}\right) \cap\left(C_{p+2}-y_{1}\right)$, such that $Y=\left\{y_{1}, y_{2}, \ldots, y_{j}\right\}$ induces a clique on heavy edges, and then find vertices $x_{2}, \ldots, x_{4-j}$ in $N\left(y_{1}\right) \cap \cdots \cap N\left(y_{j}\right) \cap\left(C_{p+1}-x_{1}\right)$ such that $X=\left\{x_{1}, \ldots, x_{4-j}\right\}$ induces a clique on heavy edges. Note that $G[X \cup Y]$ contains an element of $\mathcal{K}_{4}^{\prime}$.

We have described how to define $D_{p+1}$ and $D_{p+2}$ in the case when $q=2$ and $c_{p+1} \geqslant 0$. We now describe in general how to construct $D_{i}$ when $c_{i} \geqslant 0$. Using Claim 10.11(i), we can find, for each set in $S \in \mathcal{S}$, a set $T^{\prime}$ such that $G\left[T^{\prime} \cup S\right]$ contains an element of $\mathcal{K}_{r+1}^{\prime}$ and when we arbitrarily select a vertex $v \in S$, the set $T^{\prime} \cup(S-v)$ is $\mathcal{C}$-balanced. We let $F^{\prime}$ be the set of these arbitrarily selected vertices. Recall that when $q=2$, the set $S$ does not intersect $C_{p+2}$, so $T^{\prime} \cup(S-v)$ is actually $\mathcal{C}$-well-balanced. We label $T^{\prime} \cup S$ as $T_{v}$ and $S$ as $S_{v}$. By Claim 10.11(i), we can assume that, for every $v \in F^{\prime}$, the sets $T_{v}$ were constructed so as to be vertex-disjoint and, when $q=2$, disjoint from $X \cup Y$. For every $v \in F^{\prime}$, we add $T_{v}$ to $\mathcal{T}$. When $q=2$, using Claim 10.11(i), we find a set $T^{\prime}$, disjoint from all of the previously constructed sets, such that $G\left[T^{\prime} \cup X \cup Y\right]$ contains an element of $\mathcal{K}_{r}^{\prime}$ and $\left|T^{\prime} \cap C_{i}\right|=s_{i}$ for each $i \in[p]$, and we 
add $T^{\prime} \cup X \cup Y$ to $\mathcal{T}$. We now let

$$
D_{i}:=C_{i} \backslash F^{\prime} \text { for all } i \in[p+q],
$$

and note that for every $i \in[p]$, if $c_{i} \geqslant 0$, then $\left|D_{i}\right|=s_{i} n / r$. Furthermore, when $q \geqslant 1$ and $\left|C_{p+1} \cup C_{\sigma(p+1)}\right| \geqslant s n / r$, we have that $\left|D_{p+1} \cup D_{\sigma(p+1)}\right|=s n / r$, and, when $q=2$, we also have that both $\left|D_{p+1}\right|$ and $\left|D_{\sigma(p+1)}\right|$ are divisible by $s$.

For each vertex $v \in \bar{C}$, we use Claim 10.12(i) to find a vertex set $T_{v}$ that is $\mathcal{B}$-well-balanced and such that $G\left[T_{v}+v\right]$ contains a $\mathcal{K}_{r+1}^{\prime}$. We add $T_{v}+v$ to $\mathcal{T}$ and ensure that these sets are disjoint from the sets already in $\mathcal{T}$.

Let $F:=F^{\prime} \cup \bar{C}$ and recall that, for every $i \in[p]$ such that $\left|C_{i}\right| \leqslant s_{i} n / r$, we currently have that $D_{i}=C_{i}$. At this point, every vertex in $V$ is either in one of the sets $\left\{D_{1}, \ldots, D_{p+q}\right\}$ or is in $F$.

We now move vertices from $F$ to sets in $\mathcal{D}$ that are 'too small' until we have the desired proper ordered collection. When we do this we also make small changes to the collection $\mathcal{T}$ so that every $T \in \mathcal{T}$ will be a $\mathcal{D}$-well-balanced $r$-set.

In detail, for every $v \in F$ and $i \in[p+q]$, when we say we assign $v$ to $D_{i}$ we mean that we add $v$ to the set $D_{i}$ and update $T_{v}$ by removing one $u \in C_{i}$ from $T_{v}$ and add $v$ to $T_{v}$. This is only well defined when there is initially some $u \in C_{i}$ in $T_{v}$. Note that in this case the updated version of $T_{i}$ is $\mathcal{D}$-well-balanced. If initially $T_{v}$ contains no element from $C_{i}$ then $q=2, i \in\{p+1, p+2\}$, and $T_{v}$ intersects $C_{\sigma(i)}$ instead of $C_{i}$. Furthermore, since we are moving a vertex to either $C_{p+1}$ or $C_{p+2}$ from $F$, it must be that $\left|C_{p+1} \cup C_{p+2}\right|<s n / r$, and so if $v \in F^{\prime}$ then $S_{v} \subseteq C_{i}$ for some $i \in[p]$ such that $c_{i}>0$. In this case when we assign $v$ to $D_{i}$ we instead complete the following process: We first remove $T_{v}$ from $\mathcal{T}$. Then, if $v \in \bar{C}$, we use Claim 10.12(ii), to find a set $T^{\prime} \subseteq B \backslash V(\mathcal{T})$ such that $G\left[T^{\prime}+v\right]$ contains an element of $\mathcal{K}_{r}^{\prime},\left|T^{\prime} \cap B_{j}\right|=s_{j}$ for every $j \in[p],\left|T^{\prime} \cap B_{i}\right|=s-1$, $\left|T^{\prime} \cap B_{\sigma(i)}\right|=0$. Then let $T_{v}:=T^{\prime}+v$ and add it $\mathcal{T}$. Similarly, when $v \in F^{\prime}$, we use Claim 10.11(ii) to find a set $T^{\prime} \subseteq C \backslash V(\mathcal{T})$ such that $G\left[T^{\prime} \cup S_{v}\right]$ contains an element of $\mathcal{K}_{r}^{\prime},\left|T^{\prime} \cap C_{k}\right|=0$ where $k \in[p]$ such that $S_{v} \subseteq C_{k},\left|T^{\prime} \cap C_{j}\right|=s_{j}$ for every $j \in[p]-k,\left|T^{\prime} \cap C_{i}\right|=s-1,\left|V\left(T^{\prime}\right) \cap C_{\sigma(i)}\right|=0$, and let $T_{v}:=T^{\prime} \cup S_{v}$ and add it $\mathcal{T}$. We then add $v$ to $D_{i}$. Note that now $T_{v}$ is $\mathcal{D}$-well-balanced.

For any $i \in[p+q]$ such that $c_{i}<0$, we then arbitrarily assign exactly $-c_{i}$ of the remaining vertices in $F$ to $D_{i}$, except when $q=2$ and $i \in\{p+1, p+2\}$. When $q=2$ and $\left|C_{p+1} \cup C_{p+2}\right|<s n / r$, we again have to be careful to ensure that, in the end, both $\left|D_{p+1}\right|$ and $\left|D_{p+2}\right|$ are divisible by $s$. Therefore, assume that, when $q=2$, we assign vertices from $F$ to $D_{p+1}$ and $D_{p+2}$ before we assign vertices in $F$ to any $D_{i}$ for $i \leqslant p$. Also, note that, because $\left|D_{p+1} \cup D_{p+2}\right|<s n / r,|F| \geqslant 1$. To help us organize the assignment of vertices, we let $j \in\{p+1, p+2\}$, so that if we let $1 \leqslant k_{j}, k_{\sigma(j)} \leqslant s$ be such that

$$
k_{j} \equiv\left|D_{j}\right| \quad \text { and } \quad k_{\sigma(j)} \equiv\left|D_{\sigma(j)}\right| \quad(\bmod s),
$$


then $k_{\sigma(j)} \leqslant k_{j}$. If $|F| \geqslant s-k_{j}$, we assign $s-k_{j}$ vertices in $F$ to $D_{j}$ and then assign vertices from $F$ to $D_{\sigma(j)}$ until $\left|D_{p+1} \cup D_{p+2}\right|=s n / r$. Otherwise, we can assume $1 \leqslant|F|<s-k_{j}$, which implies $s=4$, and $|F| \leqslant 2$. Note that if $|F|=1$, then $\left|F \cup D_{p+1} \cup D_{p+2}\right|=4 n / r$, so $k_{j}+k_{\sigma(j)}=3$. Therefore, exactly one of the two following conditions must hold:

(i) $|F|=2$ and $k_{j}=k_{\sigma(j)}=1$; $\quad$ or $\quad$ (ii) $|F|=1, k_{j}=2$ and $k_{\sigma(j)}=1$.

In either case, we arbitrarily pick $v \in F$, add $v$ to $D_{\sigma(j)}$ and delete $T_{v}$ from $\mathcal{T}$. We then use Claim 10.12(ii) (if $v \in \bar{C}$ ) or Claim 10.11(ii) (if $v \in F^{\prime}$ ), to construct a $\mathcal{D}$-balanced set $T_{v}$ containing $v$ that has one vertex in $C_{\sigma(j)}$ and two vertices in $C_{j}$. We then move both of the vertices in $T_{v} \cap D_{j}$ from $D_{j}$ to $D_{\sigma(j)}$. In both cases, we now have that $\left|D_{\sigma(j)}\right|$ is divisible by 4 , and $T_{v}$ is $\mathcal{D}$-well-balanced. We then assign the possibly one remaining vertex in $F$ to $D_{j}$, so $\left|D_{j}\right|$ is divisible by 4 as well.

For every $i \in[p+q]$, we let $D_{i}^{\prime}=D_{i} \backslash V(\mathcal{T})$, and note that $D_{i}^{\prime} \subseteq C_{i}$. Therefore, if $G^{\prime}=G-V(\mathcal{T})$, then $\mathcal{D}^{\prime}=\left(D_{1}^{\prime}, \ldots, D_{p+q}^{\prime}\right)$ is a proper ordered collection of $G^{\prime}$, so, by Claim 10.10 there exists a perfect $\mathcal{K}_{r}^{\prime}$-tiling $\mathcal{T}^{\prime}$ of $G^{\prime}$, and $\mathcal{T} \cup \mathcal{T}^{\prime}$ is a perfect $\mathcal{K}_{r}^{\prime}$-tiling of $G$.

\section{Acknowledgements}

The first author is supported by Simons Foundation Grant \#521777. The second author is supported by Simons Foundation Grant \#283194. The third author is supported by NSF Grant DMS-1500121. The fourth author is supported by EPSRC grant EP/M016641/1. This research was partially carried out while the last three authors were visiting the Institute for Mathematics and its Applications at the University of Minnesota. The authors would like to thank the institute for the nice working environment. The last two authors were also supported by the BRIDGE strategic alliance between the University of Birmingham and the University of Illinois at Urbana-Champaign, as part of the 'Building Bridges in Mathematics' BRIDGE Seed Fund project. The work for this paper was carried out while the third author was a postdoctoral researcher at the University of Illinois at Urbana-Champaign. The authors are also grateful to the referees for their helpful reviews.

\section{References}

[1] N. Alon and R. Yuster, 'Almost $H$-factors in dense graphs', Graphs Combin. 8 (1992), 95-102.

[2] J. Balogh, A. Lo and T. Molla, 'Transitive triangle tilings in oriented graphs', J. Combin. Theory B 124 (2017), 64-87.

[3] F. S. Benevides, T. Łuczak, A. Scott, J. Skokan and M. White, 'Monochromatic cycles in 2-coloured graphs', Combin. Probab. Comput. 21 (2012), 57-87. 
[4] W. G. Brown and F. Harary, 'Extremal digraphs', Coll. Math. Soc. J. Bolyai 4 (1969), 135-198.

[5] K. Corrádi and A. Hajnal, 'On the maximal number of independent circuits in a graph', Acta Math. Acad. Sci. Hungar. 14 (1964), 423-439.

[6] A. Czygrinow, L. DeBiasio, H. A. Kierstead and T. Molla, 'An extension of the HajnalSzemerédi theorem to directed graphs', Combin. Probab. Comput. 24 (2015), 754-773.

[7] A. Czygrinow, H. A. Kierstead and T. Molla, 'On directed versions of the Corrádi-Hajnal Corollary', European J. Combin. 42 (2014), 1-14.

[8] C. Grosu and J. Hladký, 'The extremal function for partial bipartite tilings', European J. Combin. 33 (2012), 807-815.

[9] A. Hajnal and E. Szemerédi, 'Proof of a conjecture of Erdős', in Combinatorial Theory and its Applications II, (eds. P. Erdős, A. Rényi and V. T. Sos) Colloq. Math. Soc. J. Bolyai 4 (North-Holland, Amsterdam, 1970), 601-623.

[10] F. Havet and S. Thomassé, 'Oriented hamiltonian paths in tournaments: A proof of Rosenfeld's conjecture', J. Combin. Theory B 78 (2000), 243-273.

[11] P. Hell and D. G. Kirkpatrick, 'On the complexity of general graph factor problems', SIAM J. Comput. 12 (1983), 601-609.

[12] P. Keevash and R. Mycroft, 'A multipartite Hajnal-Szemerédi theorem', J. Combin. Theory B 114 (2015), 187-236.

[13] P. Keevash and B. Sudakov, 'Triangle packings and 1-factors in oriented graphs', J. Combin. Theory B 99 (2009), 709-727.

[14] H. A. Kierstead and A. V. Kostochka, 'An Ore-type theorem on equitable coloring', J. Combin. Theory B 98 (2008), 226-234.

[15] H. A. Kierstead and A. V. Kostochka, 'A short proof of the Hajnal-Szemerédi theorem on equitable coloring', Combin. Probab. Comput. 17 (2008), 265-270.

[16] J. Komlós, 'Tiling Turán theorems', Combinatorica 20 (2000), 203-218.

[17] J. Komlós, G. Sárközy and E. Szemerédi, 'Proof of the Alon-Yuster conjecture', Discrete Math. 235 (2001), 255-269.

[18] J. Komlós and M. Simonovits, 'Szemerédi's Regularity Lemma and its applications in graph theory', in Combinatorics, Paul Erdôs is Eighty II, (eds. D. Miklós, V. T. Sós and T. Szőnyi) Bolyai Society Studies 2 (Budapest, 1996), 295-352.

[19] D. Kühn and D. Osthus, 'Critical chromatic number and the complexity of perfect packings in graphs', in 17th ACM-SIAM Symposium on Discrete Algorithms (SODA, 2006), 851-859.

[20] D. Kühn and D. Osthus, 'The minimum degree threshold for perfect graph packings', Combinatorica 29 (2009), 65-107.

[21] D. Kühn and D. Osthus, 'Embedding large subgraphs into dense graphs', in Surveys in Combinatorics, (eds. S. Huczynska, J. D. Mitchell and C. M. Roney-Dougal) London Mathematical Society Lecture Note Series, 365 (Cambridge University Press, Cambridge, 2009), 137-167.

[22] A. Lo and K. Markström, ' $F$-factors in hypergraphs via absorption', Graphs Combin. 31 (2015), 679-712.

[23] T. Molla, 'Tiling directed graphs with cycles and tournaments', PhD Thesis, Arizona State University, Tempe, Arizona, 2013.

[24] V. Rödl, A. Ruciński and E. Szemerédi, 'A Dirac-type theorem for 3-uniform hypergraphs', Combin. Probab. Comput. 15 (2006), 229-251.

[25] E. Szemerédi, 'Regular partitions of graphs', in Problémes Combinatoires et Théorie des Graphes, Colloques Internationaux CNRS, 260 (Univ. Orsay, Orsay, 1976), 399-401. 
[26] A. Treglown, 'A note on some oriented graph embedding problems', J. Graph Theory 69 (2012), 330-336.

[27] A. Treglown, 'On directed versions of the Hajnal-Szemerédi theorem', Combin. Probab. Comput. 24 (2015), 873-928.

[28] A. Treglown, 'A degree sequence Hajnal-Szemerédi theorem', J. Combin. Theory B 118 (2016), 13-43.

[29] H. Wang, 'Independent directed triangles in a directed graph', Graphs Combin. 16 (2000), 453-462.

[30] R. Yuster, 'Tiling transitive tournaments and their blow-ups', Order 20 (2003), 121-133.

[31] Y. Zhao, 'Recent advances on Dirac-type problems for hypergraphs', in Recent Trends in Combinatorics, The IMA Volumes in Mathematics and its Applications, 159 (Springer, New York, 2015), Vii 706. 